\title{
The Rotterdam Study: 2016 objectives and design update
}

\author{
Albert Hofman $^{1}$ - Guy G. O. Brusselle ${ }^{1,2}$ - Sarwa Darwish Murad ${ }^{3,4}$. \\ Cornelia M. van Duijn ${ }^{1}$ - Oscar H. Franco ${ }^{1,5} \cdot$ André Goedegebure $^{6}$. \\ M. Arfan Ikram ${ }^{1,7,8}$ - Caroline C. W. Klaver ${ }^{1,9}$ - Tamar E. C. Nijsten ${ }^{10}$. \\ Robin P. Peeters ${ }^{3}$ Bruno H. Ch. Stricker ${ }^{1,3} \cdot$ Henning W. Tiemeier ${ }^{1,11}$. \\ André G. Uitterlinden ${ }^{1,3} \cdot$ Meike W. Vernooij ${ }^{1,8}$
}

Received: 6 July 2015/ Accepted: 2 September 2015/Published online: 19 September 2015

(C) The Author(s) 2015. This article is published with open access at Springerlink.com

\begin{abstract}
The Rotterdam Study is a prospective cohort study ongoing since 1990 in the city of Rotterdam in The Netherlands. The study targets cardiovascular, endocrine, hepatic, neurological, ophthalmic, psychiatric, dermatological, otolaryngological, locomotor, and respiratory diseases. As of 2008, 14,926 subjects aged 45 years or over comprise the Rotterdam Study cohort. The findings of the Rotterdam Study have been presented in over 1200 research articles and reports (see www.erasmus-epidemiol ogy.nl/rotterdamstudy). This article gives the rationale of the study and its design. It also presents a summary of the major findings and an update of the objectives and methods.
\end{abstract}

Keywords Biomarkers - Cardiovascular diseases - Cohort study · Dermatological diseases - Endocrine diseases . Epidemiologic methods $\cdot$ Genetic epidemiology $\cdot$ Liver diseases · Neurological diseases · Oncology · Ophthalmic diseases - Otolaryngological diseases .

Albert Hofman

a.hofman@erasmusmc.nl

1 Department of Epidemiology, Erasmus Medical Center, PO Box 2040, 3000 CA Rotterdam, The Netherlands

2 Department of Pulmonary Diseases, Erasmus Medical Center, Rotterdam, The Netherlands

3 Department of Internal Medicine, Erasmus Medical Center, Rotterdam, The Netherlands

4 Department of Gastro-Enterology, Erasmus Medical Center, Rotterdam, The Netherlands

5 Department of Cardiology, Erasmus Medical Center, Rotterdam, The Netherlands

6 Department of Otolaryngology, Erasmus Medical Center, Rotterdam, The Netherlands
Pharmacoepidemiology $\cdot$ Renal diseases $\cdot$ Psychiatric diseases $\cdot$ Respiratory diseases

\section{Introduction}

The Rotterdam Study was designed in the mid-1980s as a response to the demographic changes that were leading to an increase of the proportion of elderly people in most populations [1]. It was clear that this would produce a strong rise in elderly people living with diseases, as most diseases cluster at the end of life, and that to discover the causes of diseases in the elderly one would have to study risk factors of those diseases [2]. A major approach to finding causes is the prospective follow-up study, which has proven quite effective in finding causes of heart disease and cancer.

7 Department of Neurology, Erasmus Medical Center, Rotterdam, The Netherlands

8 Department of Radiology, Erasmus Medical Center, Rotterdam, The Netherlands

9 Department of Ophthalmology, Erasmus Medical Center, Rotterdam, The Netherlands

10 Department of Dermatology, Erasmus Medical Center, Rotterdam, The Netherlands

11 Department of Psychiatry, Erasmus Medical Center, Rotterdam, The Netherlands 


\section{The design of the Rotterdam Study}

The design of the Rotterdam Study is that of a prospective cohort study among, initially, 7983 persons living in the well-defined Ommoord district in the city of Rotterdam in The Netherlands (78 \% of 10,215 invitees). They were all 55 years of age or over and the oldest participant at the start was 106 years [3]. The study started with a pilot phase in the second half of 1989. From January 1990 onwards participants were recruited for the Rotterdam Study. Figure 1 gives a diagram of the various cycles in the study. In In 2000, 3011 participants (out of 4472 invitees) who had become 55 years of age or moved into the study district since the start of the study were added to the cohort. In 2006 a further extension of the cohort was initiated in which 3932 subjects were included, aged 45-54 years, out of 6057 invited, living in the Ommoord district. By the end of 2008, the Rotterdam Study therefore comprised 14,926 subjects aged 45 years or over $[4,5]$. The overall response figure for all three cycles at baseline was $72.0 \%(14,926$ of 20,744).

The participants were all examined in some detail at baseline. They were interviewed at home $(2 \mathrm{~h})$ and then had an extensive set of examinations (a total of $5 \mathrm{~h}$ ) in a specially built research facility in the centre of their district. These examinations focused on possible causes of invalidating diseases in the elderly in a clinically state-ofthe-art manner, as far as the circumstances allowed. The emphasis was put on imaging (of heart, blood vessels, eyes, skeleton and later brain) and on collecting body fluids that enabled further in-depth molecular and genetic analyses. These examinations were repeated every 3-4 years in characteristics that could change over time. And so there were examination cycles from 1990 to 1993 , from 1993 to 1995, from 1997 to 1999 , from 2000 to 2001 , from 2002 to 2004, from 2004 to 2005, from 2006 to 2008, from 2009 to 2011, from 2011 to 2012, from 2012 to 2014, from 2014 to 2015, and from 2015 onwards (Fig. 1). In January 2016 the fourth examination cycle for the second cohort (RS-II-4) will be finished. In February 2016 a fourth cohort will be established. The age range for this new cohort is predominantly $40-55$ years, the anticipated number of participants is 4000 .

The participants in the Rotterdam Study are followed for a variety of diseases that are frequent in the elderly: coronary heart disease, heart failure and stroke, Parkinson disease, Alzheimer disease and other dementias, depression and anxiety disorders, macular degeneration and glaucoma, respiratory diseases, liver diseases, diabetes mellitus, osteoporosis, dermatological diseases and cancer.

The Rotterdam Study has been approved by the institutional review board (Medical Ethics Committee) of the

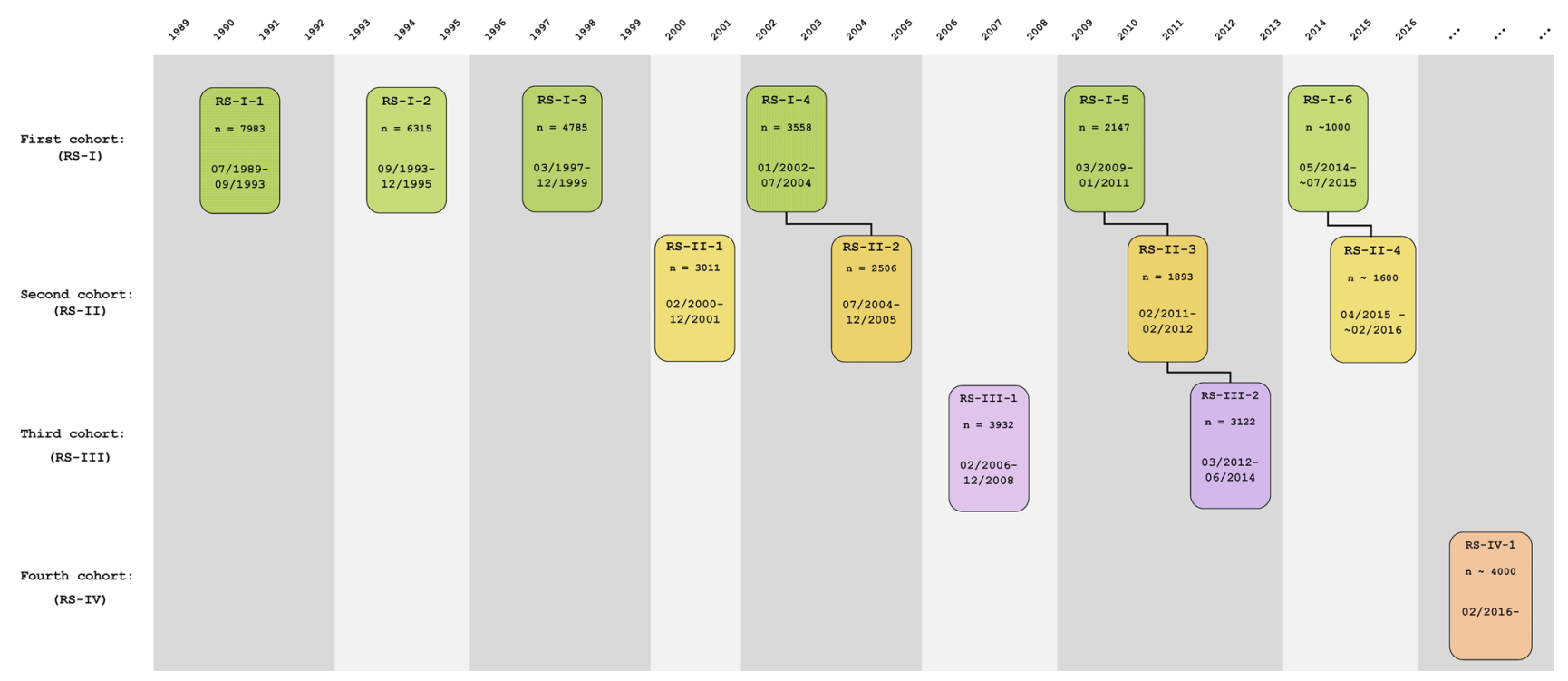

Fig. 1 Diagram of examination cycles of the Rotterdam Study (RS). RS-I-1 refers to the baseline examination of the original cohort (pilot phase 07/1989-12/1989; cohort recruitment 01/1990-09/1993). RS-I2, RS-I-3, RS-I-4, RS-I-5, and RS-I-6 refer to re-examinations of the original cohort members. RS-II-1 refers to the extension of the cohort with persons in the study district that became 55 years since the start of the study or those of 55 years or over that migrated into the study district. RS-II-2, RS-II-3, and RS-II-4 refer to re-examinations of the extension cohort. RS-III-1 refers to the baseline examination of all persons aged 45 years and over living in the study district that had not been examined already (i.e., mainly comprising those aged 45-60 years). RS-III-2 refers to the first re-examination of this third cohort. Examination RS-I-4 and RS-II-2 were conducted as one project and feature an identical research program. Similarly, examinations RS-I-5, RS-II-3, and RS-III-2 share the same program items. Also, examinations RS-I-6 and RS-II-4 are conducted as one project. RS-IV-1 refers to the baseline visit of a new cohort, to be established in February 2016 
Erasmus Medical Center and by the review board of The Netherlands Ministry of Health, Welfare and Sports. The approval has been renewed every 5 years, as well as with the introduction of major new elements in the study (e.g., MRI investigations).

In the remainder of this article the objectives and major findings will be presented with an update of the research methods for cardiovascular diseases, dermatological diseases, endocrine diseases, liver diseases, neurological diseases, ophthalmic diseases, psychiatric diseases, respiratory diseases, as well as for genetic and biomarker studies and for pharmaco-epidemiologic studies. For relevant recent EJE references see [6-28].

\section{Cardiovascular diseases}

\section{Objectives}

Research on the epidemiology of cardiovascular disease focuses on the etiology, prognosis, and prediction of cardiovascular disorders (including coronary heart disease, stroke, heart failure) diabetes mellitus and metabolic syndrome. The main emphasis is on prevention and management of a first cardiovascular event but prevention of secondary events is also an area of interest. Putative risk factors include five groups: lifestyle factors, endocrine factors, factors involved in hemostasis, inflammation and endothelial function, metabolomic factors and genetic factors. We have five specific focused themes:

1. Lifestyle: focused on evaluating the role of lifestyle factors (including nutrition, physical activity, sleep and smoking) in maintaining cardiovascular health as well as the interactions that lifestyle factors might have on other factors (e.g. genes, epigenetic marks and medications).

2. Biomarkers and genes: aimed to identify relevant biomarkers for the identification of novel mechanisms of disease. These incorporate both molecular and genetic factors together with their potential interactions. Genomics, epigenetic marks and metabolomics play a key role.

3. Prediction and women's cardiovascular health: aimed to improve the identification of individuals at increased risk of developing cardiovascular disease in order to point out windows of opportunities that could permit early preventive interventions and personalised care. A special focus is given to evaluating specific factors and formulating targeted strategies to prevent cardiovascular disease in women.

4. High risk: focused on predictors and prognosis of chronic cardiovascular conditions, like heart failure, pulmonary hypertension, and atrial fibrillation.
5. Imaging: this work theme aims to identify the contribution that new technologies can provide to the maximum benefit of early diagnosis and accurate prognosis. Major focus is on non-invasive assessment of atherosclerosis to improve the understanding of the atherosclerotic process and the prediction of cardiovascular disease, including measurement of coronary calcification with electron-beam and multi-detector CT (MDCT) and carotid plaque characterization by MRI.

\section{Major findings}

\section{Anthropometrics and cardiovascular disease}

We evaluated different anthropometric measures, including body mass index, waist circumference, waist to height ratio, waist to hip ratio and a body shape index in association with all-cause, cardiovascular and cancer mortality. We have shown that among different anthropometric measures, body shape index was strongly associated with the risk of all-cause, cardiovascular and cancer mortality [29]. Within the European Network for Genetic and Genomic Epidemiology (ENGAGE) consortium, using a mendelian randomization approach, we found that adiposity, as indicated by body mass index, has a causal relationship with coronary heart disease, heart failure and for the first time, ischemic stroke [30]. Also, there were age- and sex-specific causal effects of adiposity on cardiovascular risk factors, including cholesterol, blood pressure, fasting levels of insulin and C-reactive protein [31].

\section{Comparison of guidelines}

The new American College of Cardiology/American Heart Association (ACC/AHA) guidelines introduced a new cardiovascular (CVD) prediction model and lowered the threshold for treatment with statins to a $7.5 \%$ 10-year hard atherosclerotic cardiovascular disease (ASCVD) risk. Using 4854 asymptomatic participants from the population-based Rotterdam Study, we determined the implications of the new ACC/AHA guideline's treatment threshold and risk prediction model and compared it with the Adult Treatment Panel III (ATP-III), and the European Society of Cardiology (ESC) guidelines. We showed that proportions of individuals eligible for treatment with statins differed substantially among the 3 guidelines [32]. The ACC/AHA guideline would recommend statins for nearly all men and two-thirds of women, proportions exceeding those with the ATP-III or ESC guidelines. All risk prediction models underlying the 3 guidelines provided poor calibration and moderate to good discrimination in our population. To facilitate better clinical decision making, improving risk 
predictions and setting appropriate population-wide thresholds are necessary.

\section{Women's health}

Women experience multiple health issues throughout their life course differently from men. Therefore, attention to women's health is important in all stages in life. To improve women's quality of life and guarantee a longlasting and active role for women in society, prevention of chronic diseases and disability is a key aspect. Our focus, therefore, in the women's health group is on the major health issues for peri- and post-menopausal women, their risk factors, and prevention strategies [33].

As menopausal health is a crucial aspect in healthy and successful aging, we aimed to characterize a concept for healthy menopause. We conceptualized healthy menopause as a dynamic state, following the permanent loss of ovarian function, which is characterized by self-perceived satisfactory physical, psychological and social functioning, incorporating disease and disability, allowing the attainment of a woman's desired ability to adapt and capacity to self-manage. Conceptualization of healthy menopause serves as a crucial step in improvement of health in menopausal women, allowing for adapting adequate preventive and treatment strategies [34].

Although cardiovascular disease (CVD) remains one of the leading causes of death and disability for both men and women, our research underscores considerable sex differences in the occurrence of the various manifestations of CVD. Using the long term follow-up from the prospective population based Rotterdam Study, we showed that despite similar lifetime risks of CVD at age 55 for men and women, considerable differences in the first manifestation exist. Men are more likely to develop coronary heart disease as a first event, while women are more likely to have cerebrovascular disease or heart failure as their first event, although these manifestations appear most often at older ages [35]. Since strategies for prevention of stroke and heart failure might differ from strategies for prevention of coronary heart disease, to devise a sex-tailored primary prevention program, knowledge about the first manifestation of diseases is important.

\section{Heart failure and atrial fibrillation}

The Rotterdam Study enabled accurate assessment of the incidence and lifetime risk of heart failure and atrial fibrillation in an elderly population [36-38]. It was shown that inflammation and resting heart rate is associated with risk of heart failure [39, 40]. In addition we identified several new risk factors of atrial fibrillation. We found that markers of generalized atherosclerosis in persons without a history of myocardial infarction or angina were associated with a higher risk of atrial fibrillation [41]. Furthermore, high-normal thyroid function [42] and higher levels of dehydroepiandrosterone sulfate, a precursor in the biosynthetic pathway of androgenic and estrogenic sex hormones were associated with incidence of atrial fibrillation [43]. In collaboration with several community-based prospective studies we were able to develop a prediction model for atrial fibrillation, only using variables that are routinely collected in primary care settings [44]. In a large collaborative study as part of the CHARGE consortium, we investigated the genetic variation responsible for 6 traits related to cardiac structure and function. We found two replicated loci for left ventricular dimension and 5 replicated loci for aortic root size [45]. Another topic of interest was the search for genetic determinants of several rhythm and conduction disturbances on the ECG, notably RR-interval, QRS duration, and QT(c)-interval, PR-interval, as well as atrial fibrillation and sudden cardiac death. For example, we identified several new loci for PR interval [46], heart rate [47], and atrial fibrillation [48, 49] in metaanalyses from the CHARGE consortium.

\section{Cardiovascular risk factors and prediction}

Endocrine, inflammatory and hemostatic factors and risk of coronary heart disease were addressed in several studies. Subclinical hypothyroidism was an independent risk factor of atherosclerosis and myocardial infarction in older women [50]. In a recent study, we compared the change in the accuracy of risk predictions when newer risk markers, representative of various pathophysiologic pathways, were added to the established clinical risk predictors. Among the biomarkers, improvements in coronary heart disease risk prediction were most significant with the addition of amino-terminal pro-B-type natriuretic peptide (NTproBNP) [51, 52]. Furthermore, plasma C-Reactive protein (CRP) and lipoprotein-associated phospholipase A2 (LpPLA2) activity were independent predictors of coronary heart disease [53, 54]. Earlier findings included the association of tissue plasminogen activator (TPA) with incident coronary heart disease [55]. Using a comprehensive biomarker assay, we analysed multiple markers of inflammation among 800+ individuals with incident coronary heart disease [56]. We identified EN-RAGE as a novel biomarker for incidence of coronary heart disease, independent of established risk factors and inflammatory markers, such as C-reactive protein [56]. With respect to the prediction of coronary heart disease, EN-RAGE improved prediction significantly indicating that EN-RAGE might be useful in CHD prediction [56]. Recently, we developed and validated a coronary heart disease prediction model tailored for the aging population based on competing risk 
methodology [57]. Also, we have shown that the non-laboratory based model, based on body shape index, could predict risk of cardiovascular disease as accurately as one that relied on laboratory-based values among men [58].

\section{Non-invasive measures of atherosclerosis}

Multiple studies focused on the predictive value of noninvasive measures of atherosclerosis for risk of coronary heart disease. Strong associations with risk of coronary heart disease were found for carotid intima-media thickness [59], pulse wave velocity [60], and coronary calcification as assessed by electron-beam CT [61]. The relatively crude measures directly assessing plaques in the carotid artery and abdominal aorta predict coronary heart disease equally well as the more precisely measured carotid intimamedia thickness [62]. In persons at intermediate risk of cardiovascular disease, coronary artery calcium provided the best increment in coronary heart disease risk prediction and stratification (to reclassify persons into more appropriate coronary risk categories) [51, 63, 64]. The burden of coronary calcification also provides incremental predictive information for heart failure, but nor for cerebrovascular disease $[65,66]$.

\section{Genetic studies}

Genetic studies included candidate gene studies [67] and more recently genome-wide association studies of clinical disease and risk factor phenotypes. So far we have contributed to more than 100 Genome-wide association (GWA) studies in the field of cardiovascular disease. These GWA studies are primarily conducted in the framework of the Cohorts for Heart and Aging Research in Genomic Epidemiology (CHARGE) Consortium [68, 69] however in many instances we include further studies. We identified 3 genetic loci associated with uric acid concentration and gout [70]. Three loss-of-function variants in HAL gene were found to associate with histidine levels [71] but not with coronary heart disease. We also identified a significant association between the UMOD gene which encodes Tamm-Horsfall protein and chronic kidney disease [72]. We found four genes for systolic blood pressure, six for diastolic blood pressure and one for hypertension [73-75]. We found multiple loci that influenced erythrocyte phenotypes in the CHARGE Consortium [76]. In a metaanalysis in more than 80,000 individuals from 25 studies, we identified 18 loci for CRP levels. The study highlighted immune response and metabolic regulatory pathways involved in the regulation of chronic inflammation [77]. Novel associations of $12 \mathrm{low}$-frequency exonic variants with plasma levels of factor VII, factor VIII, and von Willebrand factor were also detected [78, 79]). The association with these variants was independent of the previously identified common variants associated with these traits, and the effect sizes were larger. We performed the first GWA study of ADAMTS13 activity, identifying independent associations with three common variants at the ADAMTS13 locus, as well as one common variant at the SUPT3H locus [80]. Additionally, we used a genotyping array focused on rare exonic variants to identify three independent rare variants in the ADAMTS13 gene associated with ADAMTS13 activity [80]. We have also identified genetic loci associated with the measures of subclinical atherosclerosis burden. Our genome-wide association studies on the 3 measures of subclinical atherosclerosis identified several new genetic loci [81-83]. We have contributed to GWA studies on coronary artery disease [84, 85]. Also, we found that 152 known coronary heart disease SNPs improved the prediction of prevalent but not incident coronary heart disease. This difference may be explained by biases related to the use of prevalent rather than incident coronary heart disease in genome-wide association studies [86]. In addition, by using genome-wide methylation data, we found an effect of tobacco smoking on DNA methylation of 12 coronary artery disease-related genes, and thus providing novel insights in the pathways that link tobacco smoking to risk of coronary artery disease [87].

Thus far, a large number of genetic variants have been identified by GWAS that contribute to the induction and development of cardio-metabolic diseases. Nevertheless, the vast majority of the identified variants map to the noncoding regions of genome that their biological relevant to the disease remain unclear. Non-coding RNAs play regulatory roles in various biological processes and cellular contexts. We identified a number functional variants in microRNA-genes and microRNA binding sites on the 3ÚTR of coding genes that affect miRNA gene regulation and explain some of the observed associations from GWAS of cardio-metabolic phenotypes [88-90].

\section{Nutrition and lifestyle}

We found that subjects who had fish intake of more than $19 \mathrm{~g} /$ day had a significantly lower prevalence of mild/moderate coronary calcification [91]. Also, we found that an increase of $50 \mathrm{~g}$ in processed meat was associated with increased CRP levels [92]. In addition to this, the intake of processed meat was associated with a higher risk of type 2 diabetes which was independent of CRP levels [92]. The dietary intake of fatty acids and its relation with CRP levels was studied as well. We found that higher intake of total PUFAs was associated with lower CRP levels and the intake of n-6 PUFAs were inversely associated with CRP [93]. We did not observe an association between n-3 PUFAs or n-3:n-6 PUFA ratio and CRP. 
Likewise, we studied whether dietary proteins, amino acids and acid load were associated with the risk of hypertension. It appeared that these factors were not a major determinant of the risk of hypertension in the Rotterdam Study [94-96]. On the other hand, we found that higher vitamin D concentrations were associated with lower prevalence of metabolic syndrome. In particular, adequate levels of vitamin $\mathrm{D}$ were associated with more beneficial HDL, triglycerides, waist circumference and serum glucose levels [97]. We did not observe an association between vitamin D and atrial fibrillation [98]. Also, we found no association between coffee consumption and incident dementia [99]. High-fat dairy was inversely related to fatal stroke, but not to incident stroke. Total dairy consumption or dairy subgroups (e.g. milk, low-fat dairy) were not associated with the occurrence of CVD events [100]. We investigated relationship of epicardial fat volume with atherosclerotic calcification volume and found that larger epicardial fat volume was related to coronary and extracranial carotid artery calcification volume in males only [101]. As part of the CHANCES consortium, we found that adherence to a healthy diet according to the WHO was associated with greater longevity [102].

Besides main effects of nutrition, we studied gene-nutrient interactions. We found that dietary vitamin $\mathrm{E}$ intake may modulate the relation of SIRT1 genetic variants with body mass index [103]. Also, we studied the modification of magnesium, whole grain and a healthy diet score on fasting glucose and insulin by SNPs related to fasting glucose and insulin as part of the CHARGE consortium [104-106]. Furthermore, low dietary carbohydrate intake and normal sleep duration were found to ameliorate cardiometabolic abnormalities conferred by common circadian-related genetic variants [107].In addition to nutrition, we also investigated lifestyle factors and found that there is no association between positive psychological well-being and incidence of cardiovascular disease [108]. We did find an association between clinical heart failure and poor sleep quality over time, but did not find an association between cardiac dysfunction (measured by echocardiography) and sleep quality [109].

\section{Methods update}

\section{Clinical follow-up}

Information on clinical cardiovascular outcomes is collected through an automated follow-up system. The followup system involves linkage of the study base to digital medical records from general practitioners in the study area and subsequent collection of letters of medical specialists and discharge reports in case of hospitalisation. With respect to the vital status of participants, information is also obtained regularly from the municipal health authorities in Rotterdam. After notification, cause and circumstances of death are established by questionnaire from the treating physicians. Clinical cardiovascular outcomes are adjudicated according to established definitions based on international guidelines by study physicians and medical specialists in the field affiliated with the Rotterdam Study. Methods of follow-up data collection, adjudication of events, and definitions of cardiovascular end points have been described in detail previously in this journal [110]. Systematic follow-up data collection is done for the occurrence of cardiovascular mortality, coronary heart disease (including coronary death, myocardial infarction, and coronary revascularization procedures), heart failure, atrial fibrillation, and sudden cardiac death [110]. Diabetes mellitus is defined based on guidelines of the American Diabetes Association and the World Health Organization. We defined incident diabetes as fasting plasma glucose level $\geq 7.0 \mathrm{mmol} / \mathrm{L}$, or the use of oral antidiabetic medication or insulin, or treatment by diet and registered by a general practitioner as having diabetes.

\section{Cardiovascular risk factors}

Besides traditional cardiovascular risk factors, five major groups of putative risk factors for cardiovascular conditions are examined. The first group are lifestyle factors, including dietary factors, physical activity, smoking, sleep and vitamin D (as described above). The second are endocrine factors, including diabetes, sex hormones, thyroid gland and adrenal gland hormones and natriuretic peptides (e.g. $[42,43,50-52])$. The third group comprises factors involved in hemostasis, inflammation and endothelial function (e.g. [53, 111, 112]). The fourth group covers genetic factors. In addition to the candidate gene approach, studies are more recently conducted through the genomewide association approach (e.g. [45-49, 70, 72-77, 81-85, 104-106, 111]). In genome-wide association studies, data from the Rotterdam Study are often combined with those from other studies in the context of the large collaborative CHARGE consortium [68, 69]. Within the fifth group we are applying both proton nuclear magnetic resonance $\left({ }^{1} \mathrm{H}\right.$ NMR) and mass spectrometry (MS) for metabolic profiling in 2000 participants of the Rotterdam Study including nearly 200 incident cases of coronary heart disease. Furthermore, in this context, special attention has been given to the contribution of different risk factors in relation to cardiovascular disease in women. Data has been collected to evaluate the impact of specific periods of potential vulnerability across a woman's lifespan; menarche, pregnancy, and menopause. Also, DNA methylation can regulate gene expression without altering the underlying DNA sequence and is now emerging as a promising molecular 
strategy for risk stratification for complex disease, including cardiovascular disease. Using the Illumina Infinium HumanMethylation450 array, we have generated DNA methylation profiles of $\sim 480,000 \mathrm{CpG}$ sites in In $\sim 1000$ samples of the RS-III.

\section{Non-invasive measures of atherosclerosis}

At baseline and follow-up examinations, ultrasonographic assessments of carotid intima-media thickness and carotid plaques were conducted in all participants [59]. At these examinations, also measurements of the ankle-brachial index and aortic calcification (on X-rays of the lumbar spine) were obtained [62]. Carotid-femoral pulse wave velocity, a measure of aortic stiffness, was measured in all participants of RS-I-3, RS-II-1, and RS-III-1 with an automatic device [60]. Measurements of coronary calcification by electron-beam CT and more recently by MDCT were conducted from 1997 onwards in RS-I and RS-II [61], [63]. From 2003 to 2006, MDCT was used to also quantify calcification in the aortic arch and carotid arteries in RS-I and RS-II. Measurement of carotid plaque components using MRI was done from 2007 to 2012 in all participants from RS-I, RS-II and RS-III with carotid wall thickening on conventional carotid ultrasound. Repeated MRI measures over time were obtained in RS-I and RS-II.

\section{Electrocardiographic, echocardiographic and other ultrasound measurements}

At every exam, a 12-lead 10-s resting ECG is made and processed by the Modular ECG Analysis System (MEANS) to obtain a series of ECG measurements [113]. Abdominal aortic diameters were measured by ultrasound at RS-I-1, and from 2002 (RS-I-4) onwards in all three Rotterdam Study cohorts. Also from 2002 onwards (RS-I4), repeated echocardiographic measurements are conducted of structural and functional left heart parameters [114]. From 2009 (RS-I-5) onwards, measurements of structure and function of the right heart are also collected, including estimates of pulmonary artery pressure. In the same round a 3-min resting ECG was measured in all participants.

\section{Nutrition and lifestyle}

Nutritional data has been collected in RS-I-1, RS-I-5, RSII-1, RS-II-3 and RS-III-1 by using semi quantitative food frequency questionnaires (FFQ). In RS-I-1 and RS- II-1, participants completed a checklist about foods and drinks they had consumed at least twice a month during the preceding year and a standardized interview using a validated 170-item semi-quantitative FFQ [115]. In RS-I-5, RS-II-3 and RS-III-I, a more comprehensive FFQ was used during the visits as described in detail previously [116-119]. Development and processing of nutrition data is being performed in close collaboration with the Department of Human Nutrition, Wageningen University, the Netherlands. In RS-I-III, RS-I-5, RS-II-I-3 and RS-III-I, physical activity data was assessed by means of an adapted version of the Zutphen Physical Activity Questionnaire and the LASA Physical Activity Questionnaire [120-122]. The questionnaire contained questions on walking, cycling, gardening, diverse sports, hobbies and on housekeeping. According to time spent in light, moderate and vigorous activity, metabolic equivalents of task were calculated. Furthermore, we are implementing objective measurement of physical activity with triaxial accelerometers in all participants.

For additional EJE references concerning cardiovascular disease see [123-182].

\section{Dermatological diseases}

\section{Objectives}

Dermatoepidemiologic research in the Rotterdam Study focuses on the frequency of the most common skin conditions as well as on genetic and environmental factors associated with these skin diseases. The emphasis is on cutaneous malignancies such as basal and squamous cell carcinomas (BCC and SCC, respectively) and their precursor lesions (actinic keratosis), inflammatory dermatoses such as eczema and psoriasis, and varicose veins. Also, we examine the frequency and determinants including genetics and environmental exposures of skin aging (pigmentation, wrinkling and photodamage) and other visible traits in collaboration with the department of Genetic Identification. Recently, we have introduced optic measures of UV exposed and non-exposed to assess whether they can function as biomarkers of skin and internal diseases.

\section{Methods}

In 2010, dermatology studies were introduced in the Rotterdam Study. To the home interview several items have been added questioning ultraviolet light exposure, history of (personal and familial) psoriasis, history of skin cancer, the diagnostic criteria of British Association of Dermatology for atopic eczema, adjusted diagnostic criteria for psoriatic arthritis. More recently, items on skin care and seborrheic dermatitis/dandruff were added.

A full body skin examination by physicians trained in dermatology with a focus on the most common skin diseases is the core contribution of dermatology. The clinical 
presence and extent of specific skin diseases (i.e., actinic keratosis, malignancies, psoriasis, seborrheic dermatitis, xerosis, hand and flexural eczema, alopecia, and signs of chronic venous insufficiency based on the ' $\mathrm{C}$ ' of the CEAP classification) at time of examination is assessed in a standardized fashion. Other dermatological diseases will just be noted.

The extent of skin aging as a global score and broken down in different aspects such as wrinkling, pigmentary spots, and teleangiecatsia are scored using a validated photonumeric scales and computer algorithms. The Norwood-Hamilton classification and the Ludwig classification is used for male and female pattern hair loss, respectively. Fully standardized 3-dimensional photographs (Premier 3dMDface3-plus UHD, Atlanta, USA) of the face are taken to further assess skin characteristics including sagging, wrinkling at different sites, teleangiectasia and pigmented spots. The colour of the facial skin and at the inner side of the upper arm are measured using a spectrophotometer (Konica Minolta Sensing, spectrophotometer CM-700d, Singapore). Recently, we have introduced optic measures of UV exposed and non-exposed skin such as relectance and fluorescence spectroscopy including the AGE reader.

As for other cancers, pathology data of the cutaneous malignancies is obtained from linkage to the national cancer registry and the Dutch pathology database (PALGA). In a further attempt to identify cohort members with psoriasis, medical files and dispenses at pharmacies have been investigated resulting in over 350 psoriasis cases.

In 2016, we aim to start collecting skin microbiome in a randomized manner among all participants. For selected skin diseases such as eczema, rosacea, seborrheic dermatosis and psoriasis the affected skin will be sampled as well.

\section{Major findings}

In the first follow-up study including the skin examinations of more than 2000 cohort members, showed that actinic keratosis is very common in this elderly population (AK prevalence was $49 \%$ for men and $28 \%$ for women) [183]. After adjusting for other factors, baldness in men was associated with a strongly increased risk of actinic keratosis.

A recent update yielded more than 1500 participants with a history of BCC, 450 with a SCC and 150 with a melanoma. We have demonstrated that approximately $30 \%$ of people with a BCC develop multiple tumors with 5 years and have developed a prediction model to identify these high risk patients. A first genetic analysis could not confirm any of the existing BCC polymorphisms to be associated with the development of multiple BCC [184]. In collaboration with researchers from the Nurses Health Study, Framingham Heart Study and deCODE, the association between common genetic variants and these skin cancers is currently being examined.

Recently, we have presented the first GWAS on actinic keratosis [185]. Several skin color genes such as IRF4, MC1R, ASIP and BCN2 were significantly associated with these premalignant skin lesions independently from skin color.

In a candidate gene study in almost 6000 people, we confirmed known and identified new variants associated with digital skin colour extraction. Of the two new skin color genes, the genetic variants in UGT1A were significantly associated with hue and variants in BNC2 were significantly associated with saturation [186]. In the International Visible Trait Genetics Consortium, we identified novel pigmentation genes confirmed by functional follow up [187]. Several pigmentation genes were also significantly associated with the presence of pigmented facial spots in a GWAS [185].

The psoriasis patients within the Rotterdam Study have predominantly mild disease. The distribution of subclinical artherosclerosis measures as well as the cardiovascular events were comparable between the 262 psoriasis patients and the reference population [188]. However, psoriasis patients were significantly more likely to have signs of nonalcoholic fatty liver disease based on ultrasonography than their controls after adjusting for potential confounders [189]. Moreover, psoriasis patients were more likely to have liver fibrosis than controls comparing Fibroscan data [190].

\section{Endocrine and locomotor diseases}

\section{Objectives}

The main objective of the programme of endocrine and locomotor epidemiology research is to study frequency and etiology of major disorders of the endocrine glands (pituitary, reproductive, thyroid, parathyroid, adrenal, and neuro-endocrine pancreas) and the musculoskeletal system and their risk factors. These include diabetes mellitus, osteoporosis, osteoarthritis, reproductive traits (fertility, age-at-menopause), and hypo- and hyper-thyroidism. The evaluation of risk factors for the above mentioned conditions includes serum measurements (such as classical hormones and other endocrine molecules), imaging of bones and joints by DXA, X-ray and pQCT, measuring muscle strength, and genetic determinants of endocrine diseases and traits. In addition, consequences of these endocrine disorders are studied in relation to aging related 
diseases, including cardiovascular disease, diabetes, eye diseases, skin diseases, and neurocognitive decline.

\section{Major findings}

In the process of obtaining digitized X-rays for all participants at all time-points of follow-up, we have evaluated the 3 major methods to score vertebral fractures: quantitative morphometry, semi-quantitative morphometry, and the qualitative ABQ method [191]. Prevalence of vertebral fractures differed substantially by the different methods, so standardization is crucial for patient care and for large scale epidemiological studies. Using the digitized X-ray scores, we have for the first time determined populationbased prevalence of Scheuermann's disease in the Dutch population to be $4 \%$ [192].

In the relationship of type 2 diabetes with bone health we observed that diabetic subjects with inadequately controlled glucose control had 1-5\% increased bone mineral density (BMD) and $\sim 50 \%$ increased fracture risk, compared to diabetics with adequately controlled glucose and to non-diabetics [193].

By studying bone health across different types of hip osteoarthritis (OA), we observed that subjects with atrophic OA (i.e., with joint space narrowing but without osteophytes) have $\sim 50 \%$ increased fracture risk as compared to controls without OA [194]. In addition, we found that hip geometry measures had modest ability to predict hip OA, in addition to clinical risk factors [195].

Over the last years, we have scored X-ray all radiographs of knee, hip and hand of RS I, II and III for osteoarthritic features including up to 20 years of followup radiographs. In addition, we have (bilateral) knee MRI images available for a subset $( \pm 1000)$ individuals of RS III, including a longitudinal follow-up MRI after 6 years. In addition, pain sensitivity measurements have been performed including a quantitative assessment of heat sensitivity on the arm using a standardized device (TSA-II neurosensory analyzer, Medoc), and indications of (widespread) pain in any part of the body using a manikin. This deep musculoskeletal phenotyping makes the Rotterdam Study a unique resource to study determinants of osteoarthritis (OA) and chronic pain and is the largest such dataset in the world. We have developed and validated (internally and externally) a prognostic model for knee osteoarthritis using clinical, genetic and biochemical risk factors [196]. We demonstrated that CTXII is the most informative biomarker for prediction of OA, while two other well-known biomarkers (COMP and C2M) are only consistently associated with prevalence of OA [197]. This indicates that COMP and C2M are merely descriptive of current OA-activity, while CTX2 has additive value for prediction of the course of disease.

We have identified associations between the presence of OA and CVD, i.e., measures of atherosclerosis [198]. However, in a longitudinal design, we observed that participants with radiographic OA were not at increased risk of CVD. Our results suggest that the close relation between disability and osteoarthritis may explain previous reports suggesting a relationship between OA and CVD [199].

Discordance between having pain and radiologic OA is a well-established fact. We have identified several factors associated to pain, which were not related to structural damage of the joints [200]. We demonstrated that type 3 finger length pattern, an indicator of prenatal androgen exposure, was associated with having symptomatic knee OA, and chronic pain [201].

We demonstrated that high-normal thyroid function is associated with an increased risk of atrial fibrillation [42]. In addition, in the first study to investigate the association between thyroid function and age-related macular degeneration (AMD), we observed an increased risk of AMD with higher values of thyroid hormone, even in the normal range of thyroid function [202]. We also for the first time identified lower TSH levels within the normal range as a risk factor for developing depression in the elderly [203].

The research line endocrine diseases is also actively involved in collaborating with economist to study the biology of economic behaviour, in particular entrepreneurial behaviour (and related traits such as educational attainment). In one of the first collaborative analyses we found no evidence for a relationship of testosteron levels with entrepreneurial behaviour [204].

Much of the work of this research is made possible by large-scale collaboration in consortia, some of which focus on one particular disease or trait (e.g., GEFOS), while others are more broad spectrum strategic collaborations (e.g., CHARGE, ENGAGE). We are part of several such large consortia studying genetic and epidemiological risk factors for osteoporosis (GEFOS, GENOMOS, CHANCES, CHARGE), osteoarthritis (TREAT-OA, ArcoGen), diabetes (MAGIC), thyroid disease (CHARGE and Thyroid Studies Collaboration (TSC)), anthropometric (GIANT) and reproductive traits (CHARGE, REPROGEN, PCOSGEN).

As part of the TSC, we recently published three individual-participant data analyses. By analyzing individual participant data from 13 prospective cohorts $(70,298$ participants) we demonstrated that subclinical hyperthyroidism is associated with an increased risk of hip and other fractures, particularly among those with TSH levels of less than $0.10 \mathrm{mIU} / \mathrm{L}$ and those with endogenous subclinical hyperthyroidism [205]. An analysis combining data from 
17 cohorts and lead by the Rotterdam Study did not show a higher risk of stroke with subclinical hypothyroidism except in participants younger than 50 years of age [206]. A combined analysis in 14 cohorts focusing on risk of coronary heart disease showed no relationship of TSH levels within the reference range and risk of $\mathrm{CHD}$ events or CHD mortality [207].

\section{Major GWAS findings}

With $>82,000$ samples collected within the GEFOS consortium a landmark publication was achieved with the identification of 56 loci influencing bone mineral density in total explaining $\sim 5 \%$ of genetic variation in BMD, and of which 14 loci also were associated with fracture risk [208]. A large GEFOS meta-analysis is currently underway to identify risk factors for fracture per se, including all types of fracture, hip fracture and vertebral fracture.

In a meta-analysis of 15,000 hip OA cases and 54,000 controls assembled in the arcOGen consortium, 5 novel loci influencing risk of hip OA were identified [209]. In an analysis of a so-called endo-phenotype of OA, i.e., joint space width narrowing (JSN) a GWAS among 10,000 subjects we identified a novel locus, called DOT1L, to influence cartilage thickness, as well as the risk for hip OA [210]. Interestingly, this gene product is a known drug target for treating leukemia with several drugs under development. Our group led a large scale meta-analysis to chronic wide spread pain in 2700 cases and 14,000 controls and identified for the first time a genetic locus involved in pain, i.e., CCT5 [211]. Within the TREAT-OA consortium, we assessed the effect of genetic variants of 199 OA candidate genes and identified a genetic variant in COL11A1 to be associated with hip OA [212]. In addition, we identified genetic loci affecting either uCTX-II or sCOMP levels, two biochemical markers for OA [213] and a novel locus for hand OA [214].

In the largest meta-analysis of GWAS of age-at-menopause among 62,000 women and which our group led, we identified 13 loci associated with differences in age-at-menopause and explaining $\sim 5 \%$ of the genetic variation [215]. Interestingly, the majority of the loci most likely involve genes involved in the DNA repair pathway which points to the importance of this system in maintaining an error-free stemcell lineage to which the oocyte belongs. As such the phenotype of age-atmenopause, represents an interesting model for age-related changes in cell function maintenance and functions as a model to identify molecular mechanisms for damage accumulation and repair during ageing.

In a meta-analysis of GWAS data on TSH levels and free T4 levels derived from up to 26,000 subjects, 26 loci were identified explaining 2-5\% of the genetic variation of TSH and fT4 respectively [216]. There was only limited overlap between the loci for TSH and fT4, and evidence was obtained for 5 loci to have sex-specific effects. A GWAS meta-analysis focusing on TPO autoantibodies (an important clinical marker for the detection of early AITD) in 16 cohorts identified five newly associated loci, three of which were also associated with clinical thyroid disease. With these markers we identified a large subgroup in the general population with a substantially increased risk of TPOAbs [217]. A follow-up study identifying 4 additional loci associated provided further insight into the genetic underpinnings of hypothyroidism. A Genetic Risk Score showed strong and graded associations with markers of thyroid function and disease in independent populationbased studies [218].

An interesting GWAS involved the analysis of Helicobacter pylori serologic status among members of the Rotterdam Study and the SHIP cohort [219]. Two novel loci were identified, TLR1 and FCGR2A, which can help explain inter-individual differences in risk for $\mathrm{H}$ pylori infection. A larger meta-analysis including several CHARGE cohorts is currently underway.

For many endocrine biomarkers GWAS have been performed to identify the genetic loci influencing their serum levels (e.g., homocysteine, testosteron, SHBG, thyroid hormone levels, etc.) and these are also involved in several mendelian randomization analyses in relation to major disease endpoints for which these biomarkers have been suggested to be predictive.

\section{Methods update}

For all participants DXA-based bone mineral density (BMD) measurements of the lumbar spine, dual hip and total body BMD, as well as determination of body composition parameters are assessed with a ProdigyTM total body fan-beam densitometer (GE Lunar Corp, Madison, WI, USA). Hip structural analysis (HSA) of DXA scans including hip strength indexes (using software by GE Lunar) is determined for all scans. All lumbar spine measurements have been scored for the trabecular bone score (TBS), Since 2009 we perform iDXA measurements (GE Lunar) which measures L1-L4 BMD, bilateral total hip and femoral neck BMD and total body BMD. From the total body scan, we measure lean mass and fat mass body composition, including total body, trunk, arm, legs, and android and gynoid regions of interest. X-ray examinations of vertebral bodies, hips, knees and hand/wrist are since 2009 obtained by a digitalized Fuji FCR system (FUJIFILM Medical Systems) for all participants. Analogue $\mathrm{X}$-ray photographs from previous time-points at all follow up measurements ( $\sim 75,000 \mathrm{X}$-rays $)$ have now all been digitized. All the X-rays have now been completely assessed for the presence of vertebral fractures and/or 
degenerative changes of the different joints (e.g., osteoarthritis, Scheuermann's Disease). Vertebral fractures and deformities are assessed using the classical quantitative McCloskey-Kanis method, the semi-quantitative Genant method, and a qualitative algorithm-based technique termed the ABQ method. Incident clinical fractures (of all bone sites) are obtained from computerized records of the general practitioners and hospital registries which are regularly checked by research physicians who review and code the fracture information. Peripheral Quantitative Computarized Tomography (pQCT-Stratec XT2000) is a low-radiation technology (low radiation dose $<1 \mathrm{uSV}$ ), which allows measuring structural properties of the bone and muscle cross section is now applied at the distal and proximal tibia (weight bearing). Measurements include geometrical parameters of volume, density of cortical and trabecular compartments and dimensions of bone and muscle. The test is done at the proximal tibia.

Muscle strength is assessed in all participants with a hand grip dynamometer. In addition jumping mechanography (Leonardo Novotec medical) has been implemented for all participants. This platform permits the dynamic examination of muscle contraction and power. In young individuals the test is performed asking participants to execute 2-3 counter movement two-footed stand (jumps) until both legs are straight and arms moving freely after receiving the instruction. The test is implemented as the "stand from a chair test" in subjects older than 85 years or with physical limitations. Muscle mass estimates are derived from whole body iDXA measurements.

The incidence and progression of OA is assessed using Kellgren scores obtained from X-rays of hips, knees, hands, and spine. The complete set of X-rays (all participants, all follow-up time points) has now been evaluated for the Kellgren score at these 4 joints. Novel diagnostic assessments for OA are available using Magnetic Resonance Imaging (MRI) of the knees in a large subset of the population ( $\mathrm{n}=\sim 1000 \mathrm{RS}-\mathrm{III}$ ). In addition, pain measurements were added in 2011 in this research line including a quantitative assessment of heat sensitivity on the arm using a standardized device, and indications of (wide-spread) pain in any part of the body using a pain puppet.

Advanced Glycation End products (AGEs) are glycooxidation products that accumulate in the body over a lifetime as part of normal ageing but formation is increased under hyperglycemic and oxidative circumstances. AGE accumulation in long-lived tissues may be associated with ageing of multiple organs and with risk to develop multiple chronic diseases Advanced glycation end-products in the skin are measured in RS-III-2, RS-I- 5 and RS-II-3 by skin autofluorescence using an AGE-Reader ${ }^{\mathrm{TM}}$ (DiagnOptics
B.V., Groningen, The Netherlands). These data are currently analyzed in relation to several diseases.

Several specific biomarker assessments in blood/serum/plasma and urine are done for the diagnosis and evaluation of risk factors of endocrine and metabolic diseases (e.g., glucose, TSH, freeT4, steroid hormones, Vitamin D, calcium, phosphate, CTXII, etc.). Fasting blood samples are collected along with challenged samples as part of a glucose tolerance test. Saliva is collected before and after a dexamethasone-suppression test. Finally, validated questionnaires evaluating nutrient intake (e.g., calcium and vitamins) and activities of daily living, allow to evaluate the role of environmental factors in endocrine conditions and locomotor diseases of the elderly.

For additional EJE references concerning endocrine and locomotor diseases see [220-242].

\section{Liver diseases}

\section{Objectives}

Fibrogenesis of the liver is most probably not only the result of well known liver diseases, such as viral hepatitis, alcoholic liver disease or non-alcoholic fatty liver disease (NAFLD), but rather a complex interaction between a genetic predisposition and these liver disorders. Liver research in the Rotterdam Study will concern the association between these known causes of liver disease and the occurrence, magnitude, and progression of fibrosis in combination with genetic and environmental factors. Additional research focus is on NAFLD. NAFLD is considered the hepatic manifestation of the metabolic syndrome and has become the most common chronic liver disease in Western countries in parallel with epidemics of obesity and type II diabetes mellitus. We aim to study the occurrence and risk factors of NAFLD in a general population and generate insight into the association with cardiovascular morbidity and mortality.

\section{Methods}

\section{Abdominal ultrasound}

From February 2009 onwards (cohorts RS-I-5, RS-II-3 and RS-III-2) onwards, trained technicians perform abdominal ultrasonography in Rotterdam Study participants. Liver, biliary tract, gall bladder, spleen, pancreas, and kidneys in combination with doppler examination of hepatic veins, hepatic artery and portal vein will be evaluated. All images are stored digitally and will be reevaluated by an ultrasound trained physician. 


\section{Assessment of steatosis}

The diagnosis and grading of liver steatosis is based on ultrasonographic liver brightness, hepatorenal echo contrast, deep attenuation and vessel blurring [243].

Non-alcoholic fatty liver disease is diagnosed by presence of steatosis on ultrasound and exclusion of excessive alcohol consumption, presence of viral hepatitis, use of known fatty liver-inducing pharmacological agents, recent bariatric surgery and a history of inflammatory bowel disease.

\section{Assessment of fibrosis}

Ultrasonographic evaluation of the liver parenchyma and liver surface is performed in order to assess severe fibrosis and/or cirrhosis. Additionally, sonographic signs of portal hypertension will be studied (splenomegaly, venous collaterals, portal vein diameter and flow, hepatic venous flow, and the presence of ascites).

To assess and quantify the grade of fibrosis trained technicians perform transient elastography in all participants by the Fibroscan. This test measures non-invasively and quantitatively the liver stiffness using a probe which includes an ultrasonic transducer transmitting a vibration wave through the liver. The velocity of the ultrasonic wave correlates directly with tissue stiffness [244, 245].

\section{Determinants of interest}

The association between factors known to influence liver function and the occurrence of steatosis and fibrosis are being studied. Additionally the association of these conditions with age, gender, nutritional intake, concurrent alcohol intake, risk factors for viral hepatitis, BMI, waistto-hip ratio, serum glucose, insulin, and diabetes mellitus, serum cholesterol and triglycerides are investigated. All clinical information is obtained by interview (updated with liver specific questions) and clinical examination. More recent efforts are focused on identifying common genetic variants associated with liver steatosis and/or fibrosis.

\section{Main findings}

We found a high prevalence of NAFLD of $35.1 \%$ within the Rotterdam Study population [246]. Main risk factors for NAFLD were found to be age, decreased physical activity lever, smoking, increased waist circumference, glucose intolerance, hypertension, and hyperlipidemia. Inversely, the risk of NAFLD seems to decrease after statin therapy [247]. Furthermore, using our ultrasound data as reference, we examined the performance of the well-known fatty liver disease index (FLI, based on waist circumference, BMI, triglyceride and gamma-glutamyltransferase (GGT) levels) in the Rotterdam Study population, and found that the FLI is a highly valid tool to predict NAFLD [248]. In another study, we found that all serum liver enzymes are related to all-cause mortality, as well as specifically cardiovascular (GGT) and cancer-related (alkaline phosphatase and aspartate aminotransferase) mortality [249]. More recently, we have examined the role of genetic factors in the multifactorial etiology of liver fibrosis, and found for example that the single nucleotide polymorphism (SNP) of the interferon gamma receptor 2, a pro-inflammatory gene known to be associated with progression to liver fibrosis in chronic hepatitis $\mathrm{C}$ patients, also was related to liver stiffness in the Rotterdam Study participants [250]. More studies are currently underway to look at other known and unknown genetic factors.

\section{Neurological diseases}

\section{Objectives}

Neuroepidemiologic research in the Rotterdam Study focuses on the frequency, etiology and early recognition of the most frequent neurologic diseases in the elderly. We study neurodegenerative diseases (dementia, including Alzheimer disease and Parkinson disease), cerebrovascular disease (both ischemic stroke and intracerebral hemorrhage), migraine and polyneuropathy. In all of these disorders clinical symptoms typically become manifest late in the disease course, the occurrence of clinical disease does not reflect the underlying spectrum of disease-related pathology, and most of the clinical syndromes are etiologically heterogeneous. Therefore, an additional research focus is on the causes and consequences of pre-symptomatic brain pathology that can be assessed with noninvasive modalities, which include MR-imaging, cognitive testing, gait assessment, and electromyography (EMG).

\section{Major findings}

Neurodegenerative and cerebrovascular diseases are highly frequent in the elderly. The prevalence increases from age 55 to 65 years to age 90 years and above from less than $1 \%$ to over $40 \%$ for dementia [251], from less than $0.5 \%$ to more than $4 \%$ for Parkinson disease [252], and from approximately $1 \%$ to nearly $10 \%$ for stroke. The incidence figures follow this pattern of a strong increase with age over the entire age range, with the age-specific incidence of dementia being identical for men and women at least until the age of 85 [253] but with men having a higher age-specific incidence of both stroke and Parkinson disease than women throughout the age range [254, 255]. 
However, more recent numbers from 2000 onwards suggest that the relative incidence of dementia and stroke may be lower than in the 1990s [256-258]. Still, in absolute numbers these disease will dramatically increase in prevalence over the coming decades.

Vascular pathology and vascular risk factors are associated with worse cognitive performance [259], which also translates in people with vascular pathology or risk factors for vascular disease having an increased risk of dementia, including Alzheimer disease [260, 261]. Moreover, several life style factors are associated with the risk of dementia and Alzheimer disease [262, 263], suggesting that onset of dementia may at least partly be delayed or prevented. However, many of these lifestyle factors have only a shortterm effect, suggesting reverse causality to some extent [264]. Commonly used drugs may also have a role in development of dementia [265]. Similar risk factor profiles also underlie cognitive decline prior to the clinical diagnosis of dementia [266, 267]. We recently investigated the total contribution that known modifiable risk factors make to total dementia burden and compared it across decades. We found that around $30 \%$ of all dementia could be prevented by effectively treating known risk factors and that this percentage had remained stable across the last two decades.

The classical risk factors for stroke also associate with the risk of stroke in the Rotterdam Study [268-271]. We have also recently shown emerging risk factors to associate with stroke [206]. We have also developed prediction rules for ischemic and hemorrhagic stroke separately [272]. Important to note is that a large amount of stroke goes clinically undetected [273]. Nearly $20 \%$ of elderly people have at least one silent brain infarct, and thereby a nearly fourfold increased risk of clinical stroke, a more than doubled risk of dementia including Alzheimer disease, and an increased risk of depression [274].

More recent work in the Rotterdam Study has been focusing on showing direct links between clinical cerebrovascular disease and dementia. In this regard, we showed subclinical heart disease to increase the risk of both stroke and dementia [275], intracranial carotid calcification to relate to both diseases [276], and subjective memory complaints to indicate a strongly increased risk of stroke [277]. With the advent of genome-wide association studies, the Rotterdam Study has contributed to large-scale collaborations and contributed to the identification of novel genes underlying the risk of Alzheimer disease and stroke [278-282].

We have also shown that although currently known genetic variants for dementia associate with cognition in non-demented elderly, these do not improve prediction [283].

Neuroimaging reveals that brain pathology is widespread [284] and can go clinically undetected for a long time. In addition to the silent infarcts, many apparently healthy elderly have ischemic changes in their cerebral white matter, i.e. white matter lesions, that are associated with an increased risk of dementia, stroke and depression. Also brain atrophy, especially of the hippocampus, is already present years before onset of even the earliest sign of cognitive impairment or subjective complaints. This emphasizes the need to shift the attention in etiologic research of neurodegenerative and cerebrovascular disease to the causes of pre-symptomatic and underlying brain changes. Technological advances in image acquisition, optimized imaging sequences and automated post-processing of multispectral MR data are major drivers of the rapid developments in this field. With our current imaging protocol we can now not only investigate established markers of brain pathology, such as infarcts, white matter lesions, and atrophy, but also extend towards novel markers, such as cerebral microbleeds and diffusion tensor imaging [285]. We have shown that risk factors profiles for subclinical disease overlap with those for clinical disease [280, 286-290]. Also, subclinical white matter lesions and silent infarcts improve the clinical prediction of incident stroke [291]. The clinical relevance of subclinical pathology is demonstrated by strong associations of these markers with morbidity and mortality [283, 292-295]. We have also extensively studied the genetic basis of subclinical brain disease, both in genome-wide settings and in candidate-gene studies [296-302] (see further section on population imaging).

Most research on the preclinical phase of neurodegenerative diseases, particularly dementia, focuses on either cognitive performance or brain pathology on neuroimaging. Given that brain pathology is usually diffuse, it is conceivable that brain functions other than cognition will also be affected. In this light, we have shown that gait deteriorates significantly with age [303]. Also, gait and cognition are linked following a specific pattern of certain cognitive domains only associated with specific aspects of gait, but not others [303]. Recently, we have reported our first findings with migraine and cerebral hemodynamis [304].

\section{Methods update}

\section{Assessment of dementia and Alzheimer disease}

In the baseline and follow-up examinations participants undergo an initial screen for dementia with the Mini Mental State Examination (MMSE) and the Geriatric Mental Schedule (GMS), followed by an examination and informant interview with the Cambridge Examination for Mental Disorders of the Elderly (CAMDEX) in screenpositives $(\mathrm{MMSE}<26$ or $\mathrm{GMS}>0$ ), and subsequent 
neurological, neuropsychological and neuroimaging examinations [251, 253]. Of subjects who cannot be reexamined in person, information is obtained from the GPs and the regional institute for outpatient mental health care. A consensus panel makes the final diagnoses in accordance with standard criteria (DSM-III-R criteria; NINCDS-ADRDA; NINDS-AIREN).

\section{Assessment of Parkinsonism and Parkinson disease}

Participants are screened in the baseline and follow-up examinations for cardinal signs of parkinsonism (resting tremor, rigidity, bradykinesia, or impaired postural reflexes). Persons with at least one sign present are examined with the Unified Parkinson's Disease Rating Scale and a further neurologic exam. PD is diagnosed if two or more cardinal signs are present in a subject not taking antiparkinsonian drugs, or if at least one sign has improved through medication, and when all causes of secondary parkinsonism (dementia, use of neuroleptics, cerebrovascular disease, multiple system atrophy, or progressive supranuclear palsy) can be excluded [252].

\section{Assessment of stroke and stroke subtypes}

History of stroke at baseline was assessed through interview and verified in medical records. Putative incident strokes get identified through the linkage of the study database with files from general practitioners, the municipality, and nursing home physicians' files, after which additional information (including brain imaging) is collected from hospital records. A panel discusses all potential strokes and subclassifies strokes into ischemic, hemorrhagic or unspecified [254, 271]. We also systematically collect transient ischemic and neurological attacks [305].

\section{Assessment of cognitive function}

Global cognitive function is measured through the Mini Mental State Examination (MMSE) in all surveys. From the third survey (RS-I-3) onwards we added a 30 min test battery that was designed to assess executive function and memory function, and which includes a Stroop test, a Letter Digit Substitution Task, a Word Fluency Test, and a 15 words Word List Learning test. This test battery was expanded from the fourth survey onwards (RS-I-4) to include motor function assessment using the Purdue Pegboard Test. Moreover, from 2009 onwards we expanded further by including the Design Orientation Test (DOT) and a modified version of the International Cooperative Ataxia Rating Scale (ICARS), which assess visuo-spatial orientation and ataxia respectively [306-308].
Assessment of gait patterns

Halfway through RS-III-1, we successfully implemented the assessment of gait in all participants using the GAITRite walkway (http://www.gaitrite.com/). Gait is assessed using a $5.79 \mathrm{~m}$ long walkway (GAITRite Platinum; CIR systems, Sparta, NJ, USA: $4.88 \mathrm{~m}$ active area; $120 \mathrm{H}$ sampling rate) with pressure sensors. Participants perform a standardized gait protocol consisting of three different walking conditions: normal walk, turning and tandem walk. In the normal walk, participants walk over the walkway at their own pace. This walk is repeated four times in both directions (yielding a total of 8 recordings). In turning, participants walk over the walkway at their own pace, turn halfway and return to the starting position (1 recording). In the tandem walk, participants walk tandem (heel-to-toe) over a line visible on the walkway (1 recording). A total of 30 spatiotemporal gait variables are calculated by the walkway software and downloaded offline for further analysis. Subsequently, principal components analysis on these thirty gait variables is performed to derive summarizing factors, referred to as gait domains. The following gait domains are used: Rhythm, Pace, Phases, Base of Support, Variability, Tandem, and Turn. Gait domains can be compared to cognitive domains, in which each domain reflects a different aspect of the overall concept [303].

\section{Assessment of polyneuropathy}

Starting in January 2013, we have successfully implemented a protocol to assess polyneuropathy. This includes a full work-up including questionnaire, neurological exam, and EMG in all participants. In coming years, we will publish on the prevalence, risk factors, and clinical correlates of polyneuropathy in the general population. The continuous measures of conductivity obtained through EMG can also serve as excellent endophenotype for genetic and biomarker studies.

\section{Rotterdam Scan Study: brain imaging within the Rotterdam Study}

In 1991, a random sample of 111 participants underwent axial T2-weighted magnetic resonance (MR) imaging to assess presence and severity of white matter lesions [284]. In 1995, a random sample of 563 non-demented participants underwent brain MR imaging in the context of the Rotterdam Scan Study. From August 2005 onwards (RS-II2 and further), a dedicated $1.5 \mathrm{~T}$ scanner is operational in the research center of the Rotterdam Study, and brain imaging is performed in all study participants without contra-indications [285]. 
Currently, the follow-up of this latter sample extends to up to 10 years (see further section on population imaging).

For additional EJE references see [99, 159, 264, 309_ 329].

\section{Ophthalmic diseases}

\section{Objectives}

Ophthalmic research in the Rotterdam Study focusses on occurrence, causally related determinants, and predictors of common eye diseases. Our main focus is on age-related macular degeneration (AMD), glaucoma and myopia, but we study retinal vessel diameters and (diabetic) retinopathy as well. In the previous studies, we mostly focused on common genetic risk variants derived from GWAS and environmental factors. We will now move on to the study of rare genetic variants, genetic effects on phenotype, in silico pathway analysis, biomarkers, and prediction models.

\section{Major findings}

\section{Age-related macular degeneration (AMD)}

AMD is one of the few complex traits of which a large proportion of the genetic background has been revealed. Currently known are 45 loci which show association with common variants, and a handful of genes which harbor rare genetic variants. Four genes have very common SNPs with major effects, and carriers of more than one of these variants have a more than $50 \%$ chance of developing AMD. Many of the identified genes play a role in the lipid pathway. Together with the 3 continent AMD consortium [330], we studied lipid genes in conjunction with serum lipid levels and incidence and progression of AMD in a total of 6950 participants. After adjustment for all confounders, we found no significant relationship between lipid levels, lipid genes, or statin-use in this meta-analysis. This points out that potential relationships between cardiovascular determinants and AMD may be indirect.

As hormones have been hypothesized to play a role in AMD, we studied thyroid-stimulating hormone (TSH) and free thyroxine (FT4) [202]. 5573 participants from the Rotterdam Study were followed for an average period of 6.9 years during which 805 persons developed AMD. Higher FT4 levels were associated with a higher risk of AMD, and participants in the highest quintile had a 1.3 fold increased risk of developing AMD. This relation was also found in euthyroid participants, indicating that this relationship exists already at a subclinical level.

Our gene-environment analyses focused on nutrition. We pooled the dietary data that we collected at baseline with data from the Australian population-based Blue Mountain Eye Study, and evaluated development of AMD in 4632 persons during 15 years of follow up [331]. For those at high genetic risk (based on carriership of the $\mathrm{CFH}$ and ARMS2 genes), we found a strongest risk reduction when their intake of lutein/zeaxanthin (risk reduction $20 \%$ ) and fish (risk reduction $40 \%$ ) was high. These data are highly clinically relevant, as they provide leads for doctors on how to advise patients at high genetic risk to alter their fate.

Direct-to-consumer companies have exploited the recent genetic advances to market genetic testing for AMD. We aimed to evaluate the merit of these companies by sending DNA samples of our own researchers to these labs, as well as to our own Rotterdam Study laboratory using our own developed prediction model [332]. The results from the companies showed a 1.6-fold difference for overall relative risk to an up to 12 -fold difference for lifetime risk. Most important reasons for the differences in risks were the testing of only a limited set of genetic markers, the choice of the reference population, and the methodology applied for risk calculation. We believe there is ample room for improvement here.

\section{Myopia (nearsightedness)}

A myopia boom is taking place all over the world. We and others found an increasing prevalence of myopia as well as a rise of the more extreme end of the spectrum, high myopia (refractive error of -6 diopters and beyond). In collaboration with studies in the European Eye Epidemiology consortium (E3), we performed a meta-analysis of refractive error prevalence [333]. We used the 2010 European Standard Population and studies ages between 25 and 90 years. The prevalence standardized to age 50 in Europe were $30.6 \%$ myopia, $2.7 \%$ high myopia, $25.2 \%$ hyperopia and $23.9 \%$ astigmatism. However, the prevalence of myopia was up to $47 \%$ in those aged 25-29 years, showing a generation increase. In the search for explanations for this strong rise, we explored the association with education [334]. We found that higher education is strongly associated with myopia, but it seems to be an additive effect and does not explain the entire frequency rise.

The clinical relevance of myopia is determined by its visual consequences. We evaluated the risk of severe visual impairment for the entire range of refractive errors, and found that high hyperopia as well as high myopia carried an increased risk of visual impairment [335]. High myopia had the most severe visual consequences. One in three high myopes developed severe visual impairment during his/her lifetime, and risks went up to $>90 \%$ for those with axial lengths of $30 \mathrm{~mm}$ and higher. The most common cause of visual impairment was irreversible myopic macular degeneration. 
In recent years, we acquired more knowledge on the genetic background of myopia. With our international CREAM consortium (Consortium of Refractive Error And Myopia), we further explored common genetic associations with axial length (AL). We replicated the association with the ZC3H11B gene [336], and found eight other genomewide significant loci in a meta-analysis. These loci were in linkage disequilibrium with the genes RSPO1, C3orf26, MIP, ZNRF3, LAMA2, GJD2, CD55, ALPPL2 and $\mathrm{ZC} 3 \mathrm{H} 11 \mathrm{~B}$. The last five were also associated with refraction, indicating significant overlap between $\mathrm{AL}$ and refractive error. In addition to $\mathrm{AL}$, we focused on the extreme ends of spherical refraction, and found 11 loci to be associated with both myopia and hyperopia [337]. The effects of the risk alleles in these loci were in opposite directions, providing support for the existence of only one refractive error trait. We also focused on astigmatism, a refractive error which results from radial inconsistencies of the cornea as well as the lens [338]. Astigmatism is a common companion of myopia and hyperopia, and is thought to have a genetic basis as well. We conducted a meta-analysis on refractive astigmatism in CREAM, and found a significant hit with a marker downstream of the neurexin-1 (NRXN-1) gene $(\mathrm{P}=3.92 \mathrm{E}-8)$. No other genome wide significant loci were found. This suggests a more complex genetic background for astigmatism than for spherical aberrations. We will continue to pursue the search for astigmatism genes the coming years.

Future research on myopia will be focused on finding the missing genes with exome analyses, whole genome analyses, and family studies; on pathway analysis and functional studies; and on development of prediction models.

\section{Primary open-angle glaucoma (POAG)}

Although many genetic risk factors for primary open-angle glaucoma (POAG) and POAG related endophenotypes have been identified these last years, a large part of the heritability is still unexplained. To this end, we combined our efforts with international study groups in the International Glaucoma Genetics Consortium (IGGC). This consortium investigated GWAS data on POAG and POAGrelated endophenotypes including intraocular pressure (IOP), vertical cup-disc ratio (VCDR), cup area (CA) and disc area (DA).

\section{Intraocular pressure}

A meta-analysis focused on GWAS of IOP was performed including 35,296 subjects from 7 countries participating in IGGC [339]. Four new IOP-associated loci were identified (FNDC3B, ABCA1, ABO, and a region on chromosome 11p11.2). Three genes also showed significant association with POAG. In a GWAS study of IOP in the Rotterdam Study I and Rotterdam Study II using 1000 Genomes imputations, we identified a new locus (ARHGEF12) for IOP [340]. We replicated this finding in 5 other populationbased studies, and found a significant association with POAG in two independent case-control studies. The ARHGEF12 gene is involved in IOP regulation via the RhoA/RhoA kinase, and novel drugs targeting this pathway show promising results.

\section{Vertical cup-disc ratio}

A meta-analysis of VCDR was performed in 27,878 individuals [341]. Ten novel loci associated with elongation of the cup were identified (COL8A1, DUSP1, EXOC2, PLCE1, ADAMTS8, RPAP3, SALL1, BMP2, HSF2 and CARD10). Persons in the highest quintile of genetic risk had a 2.5-fold increased risk of POAG compared with those in the lowest quintile. Pathway analysis implicated a negative regulation of cell growth and cellular response to environmental stress as key pathological pathways.

\section{Optic disc area parameters}

A meta-analysis investigating CA and DA in 24,089 individuals identified 10 novel loci for CA (DHRS3, TRIB2, EFEMP1, FLNB, FAM101, DDHD1, ASB7, KPNB1, BCAS3, and TRIOBP) and 10 novel loci for DA (CDC42BPA, F5, DIRC3, RARB, ABI3BP, DCAF4L2, ELP4, TMTC2, NR2F2, and HORMAD2) [342]. These loci explained an additional part of the variance in glaucoma. These results showed that $\mathrm{CA}$ and $\mathrm{DA}$ - measurements were of additional value in the identification of causal factors for glaucoma.

\section{Functional genetics of POAG}

Our previous glaucoma studies had showed that the SIX1/ SIX6 locus was associated with VCDR and POAG. Fine mapping and exome sequencing of this region revealed 2 new variants in the SIX6 gene and pointed to SIX6 as the responsible gene for the associated signal. We further aimed to investigate the functional consequences of this gene by studying the effect of a transient knockdown of SIX6 using morpholinos [343]. We found that absent function of SIX6 led to a 30-50\% decrease in eye dimensions. Histologic examination revealed an underdeveloped lens and a thin optic nerve. Expression analysis revealed up-regulation of $\mathrm{CDKN} 2 \mathrm{~B}$, a cyclin-dependent kinase inhibitor that is involved in cell cycle regulation and TGFß-induced growth inhibition that has been consistently implicated in POAG. These results confirm that SIX6 plays 
a role in eye growth and development, and that regulation of the CDKN2B protein appears to be a downstream mechanism. Future steps for POAG genetics will focus on 1000 Genomes imputations, and analysis of exome chip and exome sequencing data.

\section{POAG diagnostics and imaging}

All over the world, retinal imaging is becoming more and more a diagnostic tool for POAG. Imaging with optical coherence tomography (OCT) has made it feasible to visualize and measure the retinal ganglion cell and nerve fiber layer, two structures which are key elements in glaucoma. We collaborated with the Iowa Imaging group led by Michael Abramoff to measure the various layers of the retina using automated digital algorithms. Together with the Iowa group, we determined the screening performance of OCT layer thickness measurements in the peripapillary and macular region for POAG in the Rotterdam Study [344]. Mean thickness of the retinal ganglion cell layer in the inferior half of the macular region showed the highest predictive value (AUC $0.85 ; 95 \%$ CI $0.77-0.92$ ) and sensitivity $(53.7 \%$; $95 \%$ CI 38.7-68.0\%) for glaucoma. The mean thickness of the peripapillary retinal nerve fiber layer had AUC 0.77 (95\% CI 0.69-0.85) and sensitivity $24.4 \%$ (95\% CI 13.7-39.5\%). These data shed new light on the occurrence of glaucomatous events at the cellular level, and may indicate that the macula is more important as an initiation site than the optic disc.

\section{Methods update}

At baseline and follow-up examinations participants undergo ophthalmic measurements including best-corrected ETDRS visual acuity, refractive error, Goldmann applanation tonometry, keratometry, slit lamp examination of the anterior segment and visual field testing. In pharmacological mydriasis we make 35 color photographs of the macular area, and 20 simultaneous stereoscopic imaging of the optic disc and macular area. Since the fourth follow-up, 35 stereoscopic color photographs of the optical disc and the macular area were made (RS-I-5). Analog fundus photography was replaced by stereoscopic digital imaging of the macular area and optic disc since the third follow-up examination. Optic nerve head analysis with a Heidelberg Retina Tomograph, macular pigment density, and melanin optical density measurements were added during the third follow-up (RS-I-3). At fourth follow-up examination, fourier domain optical coherence tomography of the macular area and optical disc, axial length and width measurements of cornea, anterior chamber, lens, posterior chamber and retina measured with Lenstar; and fundus autofluorescence, infra-red and red-free measurements were added (RS-I-5).

The classification of AMD, POAG, refractive error, and retinal vessel diameters remained unchanged.

For additional EJE references see [345-349].

\section{Psychiatric diseases}

\section{Objectives}

The aim of the psychiatric research in the Rotterdam Study is to investigate the determinants, correlates and consequences of common psychiatric problems in the elderly. The focus lies on studies of depressive and anxiety disorders, sleep disturbances, addiction to smoking, and complicated grief.

\section{Study design update}

Initially, the psychiatric data collection was very limited but this was expanded strongly in the last 15 years. In the second visit most participants were screened for depressive symptoms and from the third examination onwards (RS-I3), in 1997-1999, depressive disorders have been ascertained systematically. Assessments of anxiety disorders, sleeping disturbances, and complicated grief were added in the subsequent examination (RS-I-4) and have been performed in all follow-up visits of the original and added cohorts. Recent additions to the protocol included a screening for psychotic symptoms and, from January 2012-October 2014, ambulatory polysomnography.

\section{Major determinants}

Psychiatric research in the Rotterdam Study focuses on biological risk factors. The vascular depression hypothesis was tested with different measures of atherosclerosis, arterial stiffness and cerebral blood flow [350]. We examined whether blood levels of vitamins and fatty acids, immune parameters, and markers of folate metabolism increased the likelihood of depression. Diurnal patterns of cortisol secretion were related to psychiatric and other outcomes such as subclinical atherosclerosis. Within the psychiatric research line, very few candidate gene studies were performed, whereas several GWAs were conducted in collaborative efforts focusing on depressive symptoms, sleep, anxiety and cortisol [351-354]. Several, mostly cross-sectional studies of brain morphology as possible determinants and correlates of common psychiatric disorders were completed [292]. Current data collection includes a dexamethasone suppression test to measure hypothalamic-pituitary-adrenal axis activity in all 
participants, which is unique in a population-based study. Also, psychiatric problems and psychological traits such as happiness, sleep duration, and depression are increasingly studied as determinants of health and mortality [355].

\section{Methods update}

\section{Assessment of depressive disorders and symptoms}

Information on depression is obtained from (a) psychiatric examinations, (b) self-reported histories of depression, (c) medical records, and (d) registration of antidepressant use [356]. The psychiatric examination during each visit consists of a assessment and screening with the Center for Epidemiologic Studies Depression Scale (CES-D).All screen-positive participants identified by a CESD score of 16 or above in each follow-up examination, are interviewed by a clinician (psychiatrist, psycho-geriatrician or clinical psychologist) with the semi-structured clinical interview (Dutch version of the Schedules for Clinical Assessment in Neuropsychiatry-SCAN) to diagnose depressive disorders. Major depressive disorders are classified according to the Statistical Manual of Mental Disorders, 4th revised edition (DSM-IV) criteria. To continuously monitor incidence of depression throughout follow-up, information on the occurrence of episodes of depression and depressive symptoms are continuously collected from general practitioners medical records. All medical records such as hospital discharge letters, specialists reports, and notes of the GP are extracted and copied by a research-assistants looking for potential depressive symptoms. These extracted data are rated and categorized by two medical doctors. Consensus decisions are made for disagreeing categorizations. Finally, incident episodes of MDD were defined as the first event that chronologically occurred in one of the two data sources described above.

\section{Assessment of anxiety disorders}

Anxiety disorders are diagnosed as part of the home interview. Trained lay interviewers conduct a slightly adapted version of the Munich version of the Composite International Diagnostic Interview (M-CIDI) to assess the following anxiety disorders with a computerized diagnostic algorithm according to DSM-IV criteria: generalized anxiety disorder (GAD), panic disorder, agoraphobia, social phobia and specific phobia [357]. The M-CIDI was specifically designed to obtain DSM-IV diagnoses and test-retest reliability for anxiety disorders is good (kappa for any anxiety disorder: 0.81). For all anxiety disorders, except GAD, one-year prevalence is assessed. In addition, the HADS-A is used to assess anxiety traits continuously.
Assessment of sleep and circadian rhythms

Sleep quality and disturbance is measured with the Pittsburgh Sleep Quality Index. In addition, sleep duration and fragmentation are assessed with actigraphy, a method that infers wakefulness and sleep from the presence or absence of limb movement [358]. In total, nearly 2000 persons participated in this actigraphy study: they wore an actigraph and kept a sleep diary for, on average, six consecutive nights. Follow-up assessments of actigraphic assessments in these participants are currently scored. Ambulatory polysomnographic (PSG, i.e., full sleep EEG) recordings of one night have been conducted in 940 participants. We scheduled home visits of a research assistant who placed the sensors to record an ambulant PSG (Vitaport 4; Temec, Kerkrade, the Netherlands). The PSG included six EEG channels, F3/A2, F4/A1, C3/A2, C4/A1, O1/A2, O2/A1, bilateral electrooculography, electromyography, electrocardiography, respiratory belts on the chest and abdomen, oximetry, and a nasal pressure transducer and oronasal thermocouple to measure airflow [359]. All recordings were scored according to American Association of Sleep Medicine guidelines by a registered Sleep Technologist. Recordings were manually scored in 30-s epochs for identification of sleep stages; each epoch was scored as Wake, N1, N2, N3 or REM sleep. For each sleep stage, the duration and latency was determined. In addition, we used PRANA (PhiTools, Strasbourg, France) software to automatically measure the microstructure of sleep, e.g. spindles and REM density. Polysomnography recordings are also used to calculate the apnea hypopnea index.

Circadian rhythms: Sleep-wake activity patterns over a week are studied with actigraphy as a marker of circadian rhythms. In more than 1700 persons we calculated interdaily stability, i.e. the stability of the rhythm over days and the intra-daily variability, i.e. the fragmentation of the rhythm [360].

\section{Assessment of grief}

All participants are asked if they are currently grieving. If the answer is positive we ask formal follow-up questions "When did this person die?", and "Who was this person?" The participants who answered the first question affirmatively are assessed for complicated grief with the Dutch version of the Inventory of Complicated Grief (ICG). The ICG is considered the gold standard for measurement of complicated grief in older adults because it has high internal consistency, good convergent and criterion validity. 


\section{Major findings}

Depression: The incidence and recurrence of depression in the elderly was estimated by continuously monitoring depression during a follow-up period of, on average, 8 years [356]. In total, 566 depressive syndromes and 1073 episodes of clinically relevant depressive symptoms occurred. For depressive syndromes, the incidence rate was 7.0 (95\% CI 6.0-8.3) per 1000 person-years and the recurrence rate was 27.5 (95\% CI 23.7-32.1) per 1000 person-years. The recurrence rate of depressive syndromes was equal for women and men.

In a series of studies we found some evidence for the vascular depression hypothesis. More severe coronary and extra-coronary atherosclerosis were associated with a higher prevalence of depression, as were cerebral haemodynamic changes [350]. However, our data did not support a specific symptom profile of vascular depression as previously defined. Most importantly, we found no longitudinal relation between peripheral atherosclerosis and incident depression [361]. Recently, we prospectively studied cerebral vascular risk factors such as white matter lesions, silent infarcts or blood flow in relation to depression [362]. We found evidence that small vessel disease predicted the onset of depression. This suggests that atherosclerotic processes in the brain are a specific risk factor for depression.

Sleep: We investigated the relationships of sleep duration with both cardiovascular risk factors and psychiatric disorders. We also aimed to explain sex differences in subjective and actigraphic sleep parameters [363]. If assessed by diary or interview, elderly women consistently reported shorter and poorer sleep than elderly men. In contrast, actigraphic sleep measures showed shorter and poorer sleep in men. These discrepancies were partly explained by sleep medication use and alcohol consumption. The first results using polysomnography to measure sleep EEG suggest that REM-density is a marker of depressive symptoms in the general population [359]. Other results suggest that sleep apnea and depressive symptoms are not related, although both result in fatigue [364].

Anxiety: We found that prevalent anxiety disorders fulfilling DSM-IV criteria may be much less co-morbid with depressive disorders than previously thought if the disorders are assessed with different diagnostic instruments. On the other hand, a history of depression is very common in persons with prevalent anxiety disorder [365].

Complicated grief: In our population-based study of 5741 elderly persons, current grief was reported by 1089 participants, of these 277 ( 25 or $4.8 \%$ of total) were diagnosed with complicated grief, the vast majority of which had no clinical symptoms of anxiety or depression.
Persons with complicated grief were older, had a lower level of education, and more often had lost a child [366]. Recently published work suggests that complicated grief occurs together with structural brain atrophy more often than expected by chance [367].

Genetics of common psychiatric disorders: In the past years, we have performed a series of genome-wide association studies of the above psychiatric and psychological phenotypes, mostly as part of the CHARGE consortium. Initial analyses have yielded no convincing genome wide significant results as studies were strongly underpowered, psychiatric phenotypes do not present very homogenous entities and are highly polygenetic. Disappointingly, the genome wide analyses of intermediate phenotype in the field of psychiatry such as cortisol or executive function have hardly been more successful [368, 369]. However, more recent work with larger sample sizes as part of the PGC consortium shows promising results.

Finally, ongoing psychiatric research projects examine whether and how psychological well-being or psychiatric problems contribute to survival. Most importantly, we are interested in whether the effects are specific to certain behaviour or emotions, are independent of confounding by physical disease, or can be explained by lifestyle, immunological or hormonal regulation [370].

For additional EJE references see [153, 371-384].

\section{Respiratory diseases}

Within the Rotterdam Study, the prevalence, incidence and natural history of common respiratory diseases in older subjects are investigated. The main focus of the respiratory epidemiology group is chronic obstructive pulmonary disease (COPD), but the research group also investigates acute respiratory tract infections, pneumonia, asthma, asthma and COPD overlap syndrome (ACOS) and lung cancer. In addition, the respiratory epidemiology group aims to elucidate the clinical and genetic determinants of lung function, encompassing spirometry and diffusing capacity of the lungs. Several complementary epidemiologic approaches are performed within the Rotterdam Study: clinical epidemiology, genetic epidemiology, molecular epidemiology and pharmaco-epidemiology. Thanks to an excellent collaboration with the investigators of the Generation $\mathrm{R}$ birth cohort study, also life course epidemiologic studies are performed to elucidate the early life origin of complex respiratory traits and diseases in adulthood [385].

\section{Lung function}

At each cross-sectional round of paraclinical investigations at the Ommoord research center, spirometry is performed 
using a Master Screen ${ }^{\circledR}$ PFT Pro by trained paramedical personnel according to the American Thoracic Society (ATS)/European Respiratory Society (ERS) guidelines [386.] In the most recent round of paraclinical investigations (from March 2009 till June 2014), diffusing capacity of the lungs (DLCO) has been measured in all participants of the three cohorts (RS I-5, RS II-3 and RS III-2) using the Master Screen ${ }^{\circledR}$ PFT Pro according to the ATS/ERS guideline on standardisation of the single-breath determination of carbon monoxide uptake in the lung [387]. The aim of the measurements of spirometry and diffusing capacity of the lungs is fourfold: first, to diagnose COPD according to the Global initiative for Obstructive Lung diseases (GOLD) guidelines; second, to determine the severity of disease as evidenced by the severity of airflow limitation in those subjects with an obstructive spirometry (defined as a ratio of Forced Expiratory Volume in one second (FEV1) to Forced Vital Capacity (FVC) $<70 \%$ ); third, to phenotype COPD subjects accurately by taking into account emphysema as evidenced by a decreased DLCO; and finally, to investigate the decline of lung function over time as a marker of disease activity and progression in COPD.

In collaboration with the CHARGE consortium, we have performed landmark Genome Wide Association Studies (GWAS) of lung function, which clearly implicate early life origins of adult lung function. The first GWAS of the FEV1/FVC ratio, a spirometric measure of airflow limitation (mainly due to airway obstruction), revealed eight genetic loci [388]. Interestingly, using the hypothesis-free GWAS approach, two genetic loci-near the hedgehog interacting protein (HHIP) gene on chromosome 4 and in the gene patched 1, the receptor for hedgehog, on chromosome 9-were significantly associated with airflow limitation, and appeared to be involved in the same biological pathway, namely the hedgehog pathway. This hedgehog pathway regulates the delicate intercellular communication between lung epithelial cells (derived from the endoderm) and lung mesenchymal cells such as smooth muscle cells (derived from the mesoderm) in foetal life, and is crucial for the branching morphogenesis of the lungs. In the second GWAS of FEV1/FVC in more than 90,000 subjects, thanks to a collaboration between the CHARGE and Spirometa consortia, 16 additional genetic loci have been discovered, including genes involved in oxidative stress responses (GSTD), homeostasis of extracellular matrix proteins (ADAM19, MMP15), Transforming Growth Factor- $\beta$ (TGFB) signalling (TGFB2) and branching morphogenesis (RARB) [389]. Together, these two GWAS implicate that early life events during embryonic life and infancy might contribute to lung function in adulthood. Recently, we-in collaboration with the CHARGE consortium - have published a GWAS of Force
Vital Capacity (FVC), a marker of lung volume and an independent predictor of survival in adult populations (hence the word "vita" or "life" in the term FVC) [390].

\section{Chronic obstructive pulmonary disease}

The prospective population-based Rotterdam Study is ideally suited to investigate key research questions of Chronic Obstructive Pulmonary Disease (COPD) regarding its pathogenesis, epidemiology, environmental and genetic risk factors, natural history of the disease, phenotypes, exacerbations and co-morbidities [391]. Based upon results of spirometry, medical files of general practitioners and letters of respiratory physicians, we have currently a validated diagnosis of COPD in approximately 2000 participants (out of the total cohort of 15,000 subjects), with a median followup of 12 years, implicating more than 24,000 person-years of follow-up [392]. Importantly, the COPD participants are well phenotyped, thanks to a combination of crucial informations: (1) questionnaire-based data (e.g. respiratory symptoms including shortness of breath, cough and sputum), (2) smoking history, (3) medical information on chronic diseases and acute events (based upon medical files from general practitioners and hospital letters), (4) lung function tests (spirometry and diffusing capacity of the lung), (5) exacerbation history (of both moderate exacerbations and severe exacerbations requiring hospitalisation), (6) drug dispensing data (of oral and inhaled treatments) and (7) mortality data in nearly all COPD subjects. In addition, in a large random sample of Rotterdam Study participants $(n=2500)$, CT scans of the chest have been performed for imaging of the heart, coronary arteries and mediastinal large vessels, but also providing imaging of the lungs at inspiration and the opportunity to quantify the degree and extent of emphysema.

COPD does not only affect the airways and lungs, but is also associated with extra-pulmonary manifestations and co-morbidities. In the Rotterdam Study, we have demonstrated that COPD is associated with cerebrovascular macro- and micro-angiopathy [393, 394]. In a cross-sectional analysis, COPD cases had a twofold increased prevalence of carotid artery wall thickening on ultrasonography compared with controls with normal lung function [393]. Importantly, the risk of carotid wall thickening increased significantly with severity of airflow limitation. Moreover, on magnetic resonance imaging (MRI) of the extracranial carotid arteries, lipid core plaques were more frequent in COPD subjects than in control subjects. Since lipid core plaques are more vulnerable to rupture, this might predispose COPD patients to the occurrence of stroke (especially ischemic stroke). In a second study on cerebrovascular co-morbidities in COPD, we demonstrated that subjects with COPD had a higher prevalence of 
cerebral microbleeds on MRI of the brain, which is a novel marker of cerebral small vessel disease [394]. The association was independent of age, sex, smoking status, atherosclerotic macroangiopathy, serum creatinin, total cholesterol and triglycerides. Intriguingly, regarding the specific location of the cerebral microbleeds, COPD subjects had a significantly increased prevalence of microbleeds in deep or infratentorial locations. These findings were confirmed in longitudinal analyses restricted to subjects without cerebral microbleeds at baseline, since COPD was an independent predictor of incident cerebral microbleeds in deep or intratentorial locations [394]. To what extent these imaging abnormalities contribute to the observed gait disturbances in patients with COPD [395], needs to be elucidated.

Several studies have shown that COPD is associated with cardiovascular diseases, encompassing ischemic heart disease, acute myocardial infarction, cardiac dysrhythmia (e.g. atrial fibrillation) and heart failure. Recently we have demonstrated that subjects with COPD have an increased risk of sudden cardiac death [396]. The risk increased particularly 5 years after diagnosis of COPD and in patients with frequent exacerbations of COPD. Since in observational studies in patients with COPD beta-blockers have been shown to reduce mortality, and since we have demonstrated that the effects of cardio-selective beta-blockers on pulmonary function are minimal in the general population and in COPD subjects [397], it is time to perform interventional studies with beta-blockers in patients with COPD in order to reduce the high cardiovascular and all-cause mortality in this vulnerable patient population.

Since COPD is characterized by chronic pulmonary inflammation and an accelerated ageing of the lung, we have investigated whether COPD is associated with frailty. Using the Fried criteria of (physical) frailty, evaluating nutritional status (unintentional weight loss), grip strength, mobility (slow walking speed), physical activity and exhaustion, we have determined the prevalence of frailty in 2.833 participants of the Rotterdam Study (median age: 74 years): $5.8 \%$ were frail [229]. Frail elderly had lower quality of life, had more frequently fallen or been hospitalized, and were at increased risk of dying within three years compared to non-frail elderly [229]. The prevalence of frailty in subjects with COPD (10.2\%) was significantly higher than in subjects without COPD (3.4\%). After adjusting for age, sex, smoking, corticosteroids and other confounders, COPD subjects had a more than twofold increased prevalence of frailty [395]. Importantly, COPD elderly who were frail had a significantly worse survival.

Additional EJE references [398-403].

\section{Genomics and biomarker studies}

\section{Objectives}

The team in this research line focusses on bio-banking activities of the participants of the Rotterdam Study and investigates biological determinants of disease (i.e., DNA, RNA, proteins, metabolites, microbes, etc.). Bio-banking involves collecting, storing and managing the biological tissues of participants of the Rotterdam Study at all followup measurements. This concerns mainly blood, urine, saliva, hair and faeces. We have further started to store full blood samples for the isolation of induced pluripotent stem cells. The research focus of this group concerns assessment of biological determinants of disease (biomarkers) in these biomaterials and the analysis of markers using genomic technologies (such as SNP arrays and next generation sequencing (NGS)).

\section{Major findings}

Rotterdam Study investigators are playing leading roles in several of the large global consortia focused on assessing the contribution of complex disease gene variants by prospective meta-analysis across many epidemiological cohorts, such as in CHARGE and ENGAGE, and in many disease/phenotype focused efforts such as ADSP, IGAP, PERADES, GIANT, GEFOS, REPROGEN, TREATOA, DIAGRAM, etc. Since 2005 the genome wide association study (GWAS) has changed the field of complex genetics, and identified a still growing list of common variants contributing to disease risk and explaining genetic variance of traits. While this large scale global collaboration has originated from the GWAS era, we now see similar consortia being built around the newer genomics datasets with RNA expression profiles, DNA methylation profiles, and the NGS datasets on DNA, RNA and microbiomes, including the BBMRI-NL sponsored BIOS consortium.

The Rotterdam Study has GWAS data for almost the complete dataset summing to over 12,000 DNA samples, and is involved as a major collaborative centre for metaanalysis studies of GWAS data, including national programs (RIDE, NGI-NCHA), EU-funded projects (GEFOS, TREATOA, ENGAGE), and voluntary collaborations (GIANT, MAGIC, CHARGE). Especially, from the CHARGE consortium (the Rotterdam Study together with the Framingham Study, AGES, CHS, and ARIC) many important publications have emerged on a wide variety of phenotypes and diseases from all major research lines in the Rotterdam Study [69]. You can find them discussed under the subheadings of each individual research line. 


\section{Data collection, storage and management}

At each examination at the research centre, blood, serum, plasma (citrate, heparine), and saliva is collected, as well as EDTA tubes for DNA and PAXgene tubes for RNA isolation. Fasting blood samples are collected along with challenged samples as part of a glucose tolerance test. Saliva is collected before and after a dexamethasone-suppression test. Saliva is frozen at $-196{ }^{\circ} \mathrm{C}$ before and after the challenge and stored at $-80{ }^{\circ} \mathrm{C}$. To obtain serum and plasma, tubes are centrifuged according to a protocol standardising time and conditions from the drawing of blood to centrifugation. All samples including the full blood are snap frozen at $-196{ }^{\circ} \mathrm{C}$ using liquid nitrogen and stored at $-80{ }^{\circ} \mathrm{C}$. Overnight urine samples are collected at home, frozen at $-196{ }^{\circ} \mathrm{C}$ at the research centre and stored at $-80{ }^{\circ} \mathrm{C}$.

DNA is isolated from whole blood at one laboratory at Erasmus MC by a manual salting-out protocol and is subsequently stored in Eppendorf tubes at $-20{ }^{\circ} \mathrm{C}$. A copy of the complete DNA collection of $\sim 13,000$ samples has been transferred to Matrix 2D-barcode tubes in 96-well format at another location. This copy has been subjected to normalization of DNA concentrations and made suitable for handling in 96- and 384-well micro-titer plates for subsequent downstream genomic analysis.

Starting with the RS-III round of data collection, blood drawing has also been taken place with PAXgene tubes, from which whole RNA is isolated and stored at $-80{ }^{\circ} \mathrm{C}$. This is now ongoing for the whole study population following the cycles of visits to the research centre.

Similarly, with the RS-III round, collection of faeces material has been initiated for the intestinal microbiome analysis. A collection pot is distributed at the research centre visit which is to be used at home and then by postal mailed returned to Erasmus MC where DNA is isolated and stored at $-80{ }^{\circ} \mathrm{C}$. This is now ongoing for the whole study population following the cycles of visits to the research centre.

For data management, an in-house customized samplemanagement system has been developed. All genomic data of the Rotterdam Study (e.g., SNP array, RNA expression, NGS DNA and RNA, microbiome 16S NGS) are generated in one laboratory which keeps all raw data, while QC-ed and extracted data are stored and managed in the central data repository of the Rotterdam Study.

\section{Blood serum/plasma assessments}

For all participants, serum cholesterol, HDL, LDL, triglycerides, glucose and glucose levels are assessed. In urine, micro albumin and creatinine are determined in all participants. Recently, a new "baseline" serum biomarker dataset has been generated at the Erasmus MC Clinical Chemistry Laboratory and the Endocrine Laboratory consisting of RS-I-3, RS-II-2, and RS-III-1 samples $(\mathrm{N}=\sim 10,000)$. These measurements include a steroid profile by mass-spectrometry (e.g., estrogens, androgens, cortisol), vitamin D, thyroid hormones (TSH, free T4), interleukins, CRP, IGF1, insulin, iron-parameters (iron, ferritin and transferrin saturation), fibrinogen, homocysteine, folic acid, riboflavine, pyridoxine, SAM/SAH ratio, cobalamine, Lp-PLA2, Fas/Fas-L, and abeta42/40.

\section{Human genomics facilities}

The Rotterdam Study uses one Erasmus MC laboratory (the Human Genotyping Facility, HuGE-F, at the department of Internal Medicine) for all its genomic studies on DNA, RNA, methylation, microbiome, etc. The facility use highend automated machinery including 2 Caliper pipetting robots (including a TwisterII, and integrated plate reader (OD 260/280), 2 Tecan EVO 150 Freedom pipetting robots, a Deerac Equator NS808 nanoliter liquid dispenser, PCR machines, an ABI7900HT Taqman machine (running $1 \mathrm{ng}$ gDNA in 2 microliter reactions), 2 Illumina iScan microarray readers, one Roche 450 GS Junior sequencer, two Illumina HiSeq2000 sequencing machines, and has access to Illumian HiSeq2500 and Myseq machines. DNA sample handling is centred on 384-well plates. Single SNP genotyping studies are done mostly using Taqman and Sequenom genotyping with throughputs at 30,000 genotypes per day. We work with reduced amounts of input genomic DNA of 1 nanogram per genotype. This facility has been generating all GWAS data for the Rotterdam Study as well as its RNA expression profiles, methylation profiles, and all NGS data including whole exome sequences, RNA sequencing, and microbiome $16 \mathrm{~S}$ sequencing.

\section{Genome-wide association studies (GWAS) datasets}

GWAS are based on genotyping epidemiological cohorts with high density SNP arrays with 500,000 - 5 million SNPs. The method has been shown to successfully identify common genetic factors for hundreds of traits and diseases (see www.genome.gov/GWAstudies). Through a large grant from the Dutch research organisation NWO in 2007 one of the world's largest GWAs datasets has been facilitated involving over 12,000 DNA samples from the Rotterdam Study cohorts. This GWAS dataset consists of a) a small dataset of $\sim 450$ women with $500 \mathrm{~K}$ Affymetrix arrays (Nsp250 + Sty250; the so-called pilot dataset), and b) a large dataset of $\sim 12,000$ samples covering almost all RS-I, RS-II, RS-III DNA samples consisting of $550 \mathrm{~K}$ (RSI, II; single + duo array format) and $610 \mathrm{~K}$ (RS-III; quattro array format) Illumina array genotypes. In the pilot 
dataset also other array types have been run (including Illumina Omniexpress 2.5) allowing for comparisons and 2-step imputation strategies to create local reference data [404].

The Illumina GWAS dataset of the Rotterdam Study (with approximately 500,000 SNPs having been genotyped) also forms the basis to generate so-called "imputed" datasets derived thereof. In this process the genotypes of SNPs which have been genotyped in reference datasets (such as HapMap with $\sim 8$ million SNPs genotyped), are being estimated for all Rotterdam Study samples using the basis Illumina $500 \mathrm{~K} \mathrm{SNP}$ dataset configurations in each subject. With the advent of large reference datasets becoming available based on whole genome/exome NGS, imputation activities using the Rotterdam GWAS dataset will remain an active area of development. So far, the Rotterdam Study GWAS dataset has been imputed to HapMap version 2 and 3 (with $\sim 7.5$ million resulting SNP genotypes in the Rotterdam Study dataset), and the 1000 genome dataset version 4 (with $\sim 18$ million resulting SNP genotypes in the Rotterdam Study dataset). Currently, imputations are being generated for the Genome of the Netherlands (GoNL) whole genome NGS dataset [405], and the UK10 $\mathrm{k}$ whole genome sequencing dataset.

The Rotterdam Study GWAS dataset is actively being used by all research lines within the Rotterdam Study as can be read under the subheadings of each research line in this review of the Rotterdam Study. In addition, it also serves as a control GWAS dataset for other research groups in- and outside The Netherlands for both SNP frequencies as well as copy number variations (CNVs), in which capacity it has been used in $>100$ publications up to date. Most importantly, it has formed the start of a very successful collaboration in the CHARGE consortium (combining GWAS datasets of major epidemiological cohort studies across the world) which has $>50$ phenotype working groups in which almost all research lines of the Rotterdam Study are active.

\section{Candidate gene SNP studies}

In the past, we have genotyped over 300 individual polymorphisms as part of candidate gene studies across the complete Rotterdam Study cohort using Taqman and Sequenom genotyping techniques. These mostly concern individual potentially functional SNPs per gene (e.g., ApoE), but sometimes also haplotype tagging SNPs (e.g., ESR1, ESR2, HSD11B1, fibrinogen), and also high density SNP screening (e.g., the vitamin D receptor gene). Currently, for candidate gene/SNP studies we perform lookups in the GWAS datasets and/or perform individual SNP genotyping (e.g., SNPs not on the array, badly imputed SNPs, functional SNPs).

\section{RNA expression datasets}

With the availability of good RNA from Rotterdam Study participants, starting with the RS-III subjects, studies have been initiated analysing the expression pattern of a single gene across samples or of the complete RNA collection in a sample (expression profiling). An expression profiling dataset has now been generated for, \pm 900 samples of the RS-III dataset, using the Illumina Human HT-12 v4 array containing $\sim 48,000$ probes. Subsequently, another set of \pm 900 samples have been subjected to RNA-sequencing (see below) so that a very rich expression dataset of in total \pm 1800 samples is now available. While RNA expression is known to differ between tissues, so far we only have RNA isolated from whole blood as a tissue.

\section{Methylation datasets}

In the same samples that have RNA-expression profiles (see above) we have also generated DNA methylation profiles of $\sim 480,000 \mathrm{CpG}$ sites across the human genome using the Illumina Infinium HumanMethylation450 array. As this same set of Rotterdam Study subjects was also used for the RNA expression profiling, deep genomic studies can now take place in combination with the GWAS data and NGS data in these $\sim 1800$ subjects.

\section{New developments: next generation sequencing datasets}

A major development in genomics studies has been the introduction in the past few years of high-throughput parallel sequencing methods (also known as next generation sequencing or NGS) which allow DNA (and RNA) sequencing at unprecedented high speed and low costs. This development has brought sequencing of whole genomes within reach of individual laboratories, rather than the large global effort that was needed to sequence the human genome at first pass. This development has led to a revival in Mendelian Genetics by solving many "cold cases" (because causal mutations could directly be found in a few samples, rather than by linkage analysis in many family samples and laborious sequencing analyses of dozens of candidate gene exons in the area). In addition, it has stimulated the cohort studies that had generated GWAS datasets in the past 5 years, to generate NGS data in part or all of their samples to find local/regional variants of interest and variants that are very rare. While whole genome sequencing (WGS) is often seen as the ultimate goal in these efforts, currently almost all labs turn to whole exome sequencing (WES) because it is much more cost-effective. In WES the parts that code for amino acids as part of the encoded proteins (i.e., the exons) are first captured from the whole genome by hybridization techniques upon 
which the selected parts (the exons) are then subjected to NGS. WES costs 5-10 times less euro's than WGS (so, more samples can be done with the same amount of money) and generates interpretable findings. (note that for the vast majority of areas in the human genome we have no good idea what they signify as opposed to exons that encode functionally important parts, i.e., the parts that make up the proteins).

\section{Whole exome sequencing dataset}

A large grant from the NGI-sponsored Netherlands Consortium for Healthy Ageing (NCHA), allowed to generate WES NGS data for $\sim 3000$ samples by the HuGe-F facility on the Illumina HiSeq2000 sequencing machines. The samples for this experiment were selected to constitutes a random sample from the RS-I dataset. These NGS data have been generated, and variants have been called. This process is complicated and no golden standard exists, so these steps take place together with colleagues at Baylor College, the Broad Institute, and NGS facilities in Germany and Netherlands, and is likely to be repeated several times in the coming years when new software tools and data becomes available. Through a collaborative grant from the NIH Alzheimer initiative (ADSP) we will also obtain an additional $\sim 1000$ samples with WES NGS data from RS-I. Also here we collaborate with colleagues in CHARGE since NGS data have been generated in large parts of the ARIC study, the Framingham Study, and the CHS study. Within the several working groups these data are currently being analysed in relation to specific phenotypes.

From the first NGS efforts in reference datasets using WES, many thousands of novel DNA sequence variants were discovered in the exons, mostly rare to very rare and not well-covered on the existing GWAS arrays. Illumina and Affymetrix therefore decided to generate an array devoted to analyse these new exome variants called the Infinium HumanExome array. This array has been used to genotype many DNA samples in the Netherlands (sponsored by BBMRI) and across the globe and several collaborative analyses are currently using data generated with this array. In the Rotterdam Study we generated exome array genotypes of $\sim 3000$ samples of RS-I (with version 1 of this array), and this dataset is part of several collaborative efforts, including the Dutch BBMRI exome array effort (with $\sim 35,000$ collective samples), an effort in GIANT on anthropometric traits, and the CHARGE exome array effort across many different phenotype working groups (with $\sim 60,000$ collective samples).

\section{RNA sequencing dataset}

In a large BBMRI-sponsored collaborative effort to create a large-scale data infrastructure to work on integrative omics studies in Dutch Biobanks, the Erasmus MC HuGe-F genomics facility has generated RNA sequencing profiles of in total \pm 4000 individuals of six Dutch biobanks, including the Rotterdam Study. A total number of 900 RSsamples were RNA-sequenced at a depth of 30 million paired end reads. Together with colleagues at UMCG Groningen and LUMC Leiden, the dataset was QC-ed and annotated RNA-expression profiles were generated and relations between genetics, transcriptomics, and epigenetic measures are currently being analysed.

\section{Microbiome 16S NGS dataset}

Through applying NGS, it has recently become clear that humans carry an astonishing variety and abundance of micro-organisms (microbes, viruses, yeasts, unicellular organisms) in and on their bodies, estimated to encapsulate 100 -fold more cells than a human body counts. These insights come from analysing diversity in 16S RNA sequences which are species-specific for microbes, but also from analyzing the complete microbial genomes through much deeper so-called "meta-genomics" sequencing. While microbes can be found almost everywhere in and on the body, most studies have focused on analyzing faeces/ stool samples representing the microbial flora in the (last part of the) intestinal system. Sofar, this knowledge has come from very small studies with limited phenotype information outside an epidemiological setting. While the latter approach of meta-genomics is $(10-20 \times)$ more expensive and generates vast amounts of data with concommittant challenges in mapping and data-analysis, most epidemiological studies focus now on establishing $16 \mathrm{~S}$ datasets and mostly derived from stool. HuGe-F has optimized stool collection protocols to be applied in epidemiological settings (collection at home, shipment by mail), and has introduced and optimized 16S sequencing protocols (NGS of the $16 \mathrm{~S} v 3 / \mathrm{v} 4$ area). Within the Rotterdam Study RS III, we have collected $\sim 1700$ stool samples from which DNA has been isolated and which are currently subjected to $16 \mathrm{~S}$ v3/v4 NGS analysis. For other source of microbiomes (eye, urine, mouth, skin, etc.) several pilot projects are ongoing to establish feasibility.

\section{New developments: integrative genomics}

GWAS have identified a large number of genetic risk markers for many diseases and traits over the last decade. The Rotterdam Study has been part of many of those discoveries. However, because most variants and their proxies are non-coding, it is generally difficult to identify the causal genes. Integrative genomic studies can help to identify functional mechanisms underlying the genetic associations found. By combining genetic, transcriptomic, 
and epigenetic data, driving disease mechanisms can be identified. Similarly this can be done for analyzing microbiomes in this context of genetic susceptibility and genomic variables and risk factors.

Within the Rotterdam Study, novel epigenetic and transcriptomic datasets have been generated in large subsets of the study (see above) and transcriptomic data (RNAseq/array)). Part of this data is acquired within the BIOS consortium which is rainbow project funded by BBMRI-NL to create a large-scale data infrastructure and to bring together BBMRI researchers focusing on integrative omics studies in Dutch Biobanks. Using this data, we performed the largest expression quantitative trait locus (eQTL) meta-analysis so far reported in non-transformed peripheral blood samples, including replication. We identified multiple trait-associated SNPs (diabetes, cholesterol, SLE) that affect multiple trans-genes, and identified novel insights into these associations (PMID: 24798236). Together with collaborators from UMCG Groningen, we identified cell-specific eQTL within whole blood RNA expression data without having to sort cells [406]. By studying gene expression signatures of blood pressure, we provided new insights into molecular mechanisms underlying BP regulation [407].

As part of a second rainbowproject of BBMRI a total 2981 samples has been characterized for 232 metabolites at Brainshake's Biomarker Analysis Platform (http://www. brainshake.fi/). The sample included all participants of RSI-4 (N = 2807) and 79 of RSII-2, 2 of RSII-3 and 87 of RS3-2.The metabolites comprise of small molecular compounds and lipoproteins subfractions.

For additional EJE references see [22, 26, 27, 149, 408424].

\section{Pharmaco-epidemiologic studies}

\section{Objective}

In the Rotterdam Study, the role of drugs is studied as determinant of diseases in middle-aged and older community-dwelling individuals. This includes studying efficacy and effectiveness of drugs, as well as adverse reactions to drugs. As the drugs used in the Rotterdam Study are licensed and often on the market since several years, research focuses on determinants which modify the safety and effectiveness of widely used drugs because these often have a great impact on healthcare.

\section{Major findings}

Below, we summarize findings over the most recent period. These findings can be distinguished into three topics of special interest, i.e. genetic and other determinants of effectiveness and adverse effects of statins; cardiac rhythm disorders and sudden cardiac death; and miscellaneous pharmaco-epidemiologic topics of interest in the Rotterdam Study.

\section{Statins}

The increasing incidence and prevalence of cardiovascular disease (CVD) constitutes a considerable disease burden. CVD frequently co-exists with other diseases such as type 2 diabetes mellitus (T2DM) and non-alcoholic fatty liver diseases (NAFLD), which are both strongly related to the metabolic syndrome. Statins are cholesterol-lowering drugs that are beneficial in the primary and secondary prevention of CVD. With an approximately 20-25\% reduction of the risk of major cardiovascular endpoints, these drugs have definitely entered daily clinical practice. However, some individuals may not respond adequately to statins, and noncompliance can be a problem [425]. In 2013, the American ACC/AHA guidelines on primary prevention of CVD lowered the threshold for the indication for statin treatment. According to these guidelines, we analysed in the Rotterdam Study how many people would have had an indication for statins. The ACC/AHA guideline would recommend statins for nearly all men and two-thirds of women of the Rotterdam Study, proportions exceeding those according to the ATP-III or ESC guidelines [32]. This somewhat unbelievable result shows how fast and irresistible medicalization of Western society progresses.

We investigated genetic variation that modified the efficacy and effectiveness of statins, and their risk of adverse drug reactions in clinical practice. We used the hypothesisfree genome-wide association (GWAS) approach to discover new genetic markers without a priori hypothesis of the underlying genetic variation, and the candidate gene approach to replicate genetic variation that has previously been associated with a modified statin response or occurred in a pathway that relates to statin pharmacokinetics [425]. We investigated the LDL-cholesterol lowering response to statins in a GWAS, as part of the GIST consortium including more than 40,000 statin users in both randomized controlled trials and observational studies. In this large pharmacogenetic meta-analysis, two loci at Sortilin 1 (SORT1) and SLCO1B1 were newly discovered to be associated with a stronger, and decreased LDL-cholesterol lowering response to statins, respectively. Furthermore, previously described associations with Apolipoprotein E (APOE) and Lipoprotein, $\mathrm{Lp}$ (a) (LPA) were confirmed, that showed a stronger and decreased LDL-cholesterol lowering response, respectively [426]. In three candidate gene studies, we investigated the influence of genetic polymorphisms on the cholesterollowering response to statins. First, we investigated the role 
of genetic variation in genes involved in cholesterol metabolism. We selected polymorphisms in these genes based on a hypothesis-free approach, and subsequently tested the most promising ones in a candidate gene analysis. We showed that two polymorphisms in two different genes, rs1532624 in the cholesteryl ester transfer protein (CETP) gene and rs533556 in the apolipoprotein A-I (APOA1) gene, were associated with a decreased cholesterol lowering response to statin therapy. The association for the CETP polymorphism was subsequently replicated in an independent population [427]. Second, we were the first study that demonstrated that two strongly linked polymorphisms in the peroxisome proliferator- activated receptor (PPARA) gene, rs4253728 and rs4823613, were associated with a stronger cholesterol lowering response to statins. Thereby, we confirmed a pharmacokinetic mechanism which was previously discovered, i.e. that these two polymorphisms were associated with significantly decreased cytochrome P450 3A4 (CYP3A4) enzyme expression and activity [428]. Third, we performed a first replication of a recent finding that the rs13064411 polymorphism was associated with increased statin-induced serum proprotein convertase subtilisin/kexin type 9 (PCSK9) concentrations, and cholesterol response to statins. PCSK9 binds to the low-density lipoprotein (LDL-) receptor, and subsequently promotes the receptor for degradation. We showed that the rs13064411 polymorphism was associated with a decreased cholesterol lowering response to statins, and this effect was stronger in women and in users of a high dose of statins [429]. The risk of MI is indirectly influenced by statins via cholesterol lowering, but is also influenced by underlying diseases, such as hypertension and T2DM. The heterogeneity in causal risk factors for a hard clinical endpoint such as myocardial infarction (MI), may affect the probability of detecting one specific gene-statin interaction and may therefore require more power than with an intermediate endpoint. The CYP3A4*22 polymorphism, previously associated with a stronger cholesterol lowering response to statins, was based on the magnitude of its effect on cholesterol, a good candidate to investigate on the outcome MI. However, we could not demonstrate significant effect modification by the CYP3A4*22 polymorphism on the effect of statins in reducing the risk of MI, neither in the independent UCP study and Rotterdam Study separately, nor in a meta-analysis of the two studies [430]. In patients with a body mass index (BMI) $\geq 27.5$, current use of statins for more than 2 years was significantly associated with an approximately three times lower NAFLD prevalence [247].

In general, statins are safe and well-tolerated drugs, although a common ADR is myopathy, which can vary from myalgia to life-threatening rhabdomyolysis. We confirmed the previously described association between the rs4149056 c.521T $>$ C polymorphism in the solute carrier organic anion transporting polypeptide (SLCO1B1) gene and an increased risk of developing ADRs to statins. In simvastatin users in the Rotterdam Study, we demonstrated that the rs4149056 polymorphism was associated with an increased risk of a dose decrease or switch to another cholesterol lowering drug, as indicators for ADRs. For atorvastatin users, an association was found in users with a starting dose of more than 1.00 standardized defined daily dose [431]. In another analysis in the Rotterdam Study, current use of statins was associated with lower total and (bioactive) non-sex hormone-binding globulin (SHBG)bound testosterone levels in males. Statins decrease cholesterol production, and cholesterol is a precursor in the testosterone biosynthesis pathway. This association might be clinically relevant, given the important biological role of testosterone, the increased use of statins, and the fact that a modest average decrease in a population might hide a substantial decrease in a handful of individuals and in those with an already low testosterone level [432].

\section{Drug-induced rhythm disorders and sudden cardiac death}

A prolonged QT interval is an important risk factor for ventricular arrhythmias and sudden cardiac death. In an analysis in the Rotterdam Study over the 20-year period 1990-2010, a declining incidence of sudden cardiac death was observed [433]. QT prolongation can be caused by drugs, but also other factors, such as genetic variants are important for the occurrence of QT prolongation. A genetic association study of QT interval highlighted a role for calcium signalling pathways in myocardial repolarization [434]. QT prolongation is one of the most common reasons for withdrawal of drugs from the market, despite the fact that these drugs may be beneficial for certain patients and not harmful in every patient. Identifying genetic variants associated with drug-induced QT prolongation might add to precision medicine and prevent beneficial drugs from being withdrawn unnecessarily. We reassessed the association between tricyclic antidepressants (TCAs) and prolongation of the QT interval. The rationale of this study was that the heart-rate corrected QT interval (QTc) calculated with Bazett's formula is overestimated in users of anticholinergic drugs, which also include the TCAs. In our study population, we observed that the use of TCAs was associated with a longer QTc as calculated with Bazett's formula. However, when other correction methods were used or heart rate was included as an additional covariable in the statistical model, no association between use of TCAs and QTc was observed [435]. We also assessed the association between individual SSRIs and QTc prolongation. Of the individual SSRIs, only citalopram use was associated with a prolonged QTc in our study. Even though regulatory authorities restricted the use of citalopram to 
$20 \mathrm{mg}$ in patients 60 years and older, participants aged 60 years and older in our study using citalopram dosage with a daily dose of more than $20 \mathrm{mg}$ had a longer QTc. Although our study had a limited number of citalopram users, this study may rise questions about the safety of citalopram in high-risk patients populations (e.g., elderly) [436]. A new potentially interesting risk factor for sudden cardiac death is QT variability [437].

A second important rhythm disorder is atrial fibrillation with its high prevalence and potential cerebrovascular consequences. In the Rotterdam Study, we were able to confirm earlier work on the association between use of non-steroidal anti-inflammatory drugs (NSAIDs) and atrial fibrillation [438] and its association with hypokalemia [439]. As important potential effect modifier, we participated in a collaborative study demonstrating several new risk variants for atrial fibrillation [440].

\section{Miscellaneous topics of pharmaco-epidemiologic interest}

Over the last decades, antidepressant drug use increased in Western countries, including in the Netherlands. Older adults using antidepressant drugs deserve special care, as they often have a slower metabolism of drugs, impaired renal function, more co-morbidity, and more concomitantly used medications. We investigated the utilization of antidepressants in the Rotterdam Study over the period 1991-2011. The yearly prevalence of antidepressant use increased from $3.9 \%$ in 1991 to $8.3 \%$ of the population in 2011. The increase in SSRI use was 5.8-fold, whereas use of other antidepressants doubled and TCA use remained stable over time. The incidence of all antidepressants decreased from 23.9 to 14.2 per 1000 person-years between 1992 and 2011. The duration of a first treatment episode increased over time [441]. In a further study, we demonstrated an association between genetic variation in the $\mathrm{ABCB} 1$ gene and switching, discontinuation, and dosage of antidepressant therapy: results from the Rotterdam Study [442].

Several studies have been associated with haemorrhagic strokes, notably coumarins and other anticoagulants and aspirin but less is known about an association with cerebral microbleeds on MRI. In the Rotterdam Study, we investigated the association with several drugs. Use of serotonin reuptake inhibiting antidepressants was not related to presence of cerebral microbleeds [443]. Compared with never users, coumarin users had a higher prevalence of deep or infratentorial microbleeds and a higher incidence of any microbleeds, although statistical significance was not reached in the latter. A higher maximum INR was associated with deep or infratentorial microbleeds. Among coumarin users, a greater variability in INR was associated with a higher prevalence of microbleeds [444]. In stroke- free individuals, clopidogrel use was associated with a higher prevalence and higher number of microbleeds [445]. In further studies, it was demonstrated that chronic use of coumarin anticoagulants is associated with renal function decline [446]. Although thiazide diuretics are a well-known cause of hyponatremia, hypokalemia is also common [447]. Cardioselective beta-blockers were developed to prevent respiratory adverse effects, also these drugs are associated with a decreased pulmonary function [397]. In a large international consortium meta-analysis, no association could be demonstrated between pioglitazone and bladder cancer [448].

\section{New developments}

Most new pharmaco-epidemiologic developments nowadays are associated with pharmacogenomics, proteomics and metabolomics. In an international collaborative study, we used tissue-specific quantitative interaction proteomics to map a network of five genes involved in the Mendelian disorder long QT syndrome (LQTS). We integrated the LQTS network with GWAS loci from the corresponding common complex trait, QT-interval variation, to identify candidate genes that were subsequently confirmed in Xenopus laevis oocytes and zebrafish. We used the LQTS protein network to filter weak GWAS signals by identifying single-nucleotide polymorphisms (SNPs) in proximity to genes in the network supported by strong proteomic evidence. Three SNPs passing this filter reached genomewide significance after replication genotyping [449]. As part of methodology development, we used multiple crosssectional assessments of depressive symptoms in a population-based study to identify potential genetic interactions with SSRIs as a model to study genetic variants associated with SSRI response. We used repeated measurement models to test multiplicative interaction between genetic variants and use of SSRIs on repeated CESD scores. Besides a genome-wide analysis, we also performed an analysis which was restricted to genes related to the serotonergic signalling pathway [450]. Furthermore, we demonstrated the potential for increased power using GEE analyses instead of cross-sectional analyses. To illustrate methods for detection of gene-drug interactions on a genome-wide scale, using repeated measures data, we conduct single-study analyses and meta-analyses across studies in three large cohort studies participating in the CHARGE consortium [451].

In the field of statistical modelling of drug exposure data, we extended our previous work on analysing drug use as a time-dependent exposure [452]. We performed a study in which the association between statins and cardiovascular endpoints was analysed with marginal structural modelling [453]. 
For additional EJE references see [10, 126, 128, 142, 240, 453-468].

\section{Imaging studies}

\section{Objective}

Biomedical imaging allows for non- or minimally-invasive assessment of structural and functional changes that may reflect specific pathology. Recent developments in image data acquisition and analysis enable to use these techniques on a large scale. The Population Imaging Unit within the Rotterdam Study aims to assess imaging biomarkers of disease in a pre-symptomatic phase at the population level. Advantages of imaging measures include that they mark early disease, can be assessed reliably and reproducibly, and are quantitative rather than qualitative which makes them more powerful than most conventional outcome measures such as clinical phenotypes.

The main imaging modalities that are currently being applied in the Population Imaging Unit are multidetector computed tomography (MDCT) and magnetic resonance imaging (MRI).

\section{Imaging infrastructure and storage}

\section{$M D C T$}

CT imaging is performed with hospital-based 16- or 64-slice MDCT scanners (SOMATOM Sensation 16 or 64, Siemens, Forcheim, Germany), located at Erasmus MC. Scanners are operated by clinical technicians. CT images are acquired without contrast-enhancement and according to standardized protocols. Imaging data are transferred from the CT scanner to a securely backed-up research picture archiving system.

\section{MRI}

From August 2005 onwards, a dedicated 1.5 T MRI scanner (GE Healthcare, Milwaukee, WI, USA) is operational in the Rotterdam Study research center. This scanner is operated by trained research technicians and all imaging data are collected according to standardized imaging protocols. Changes or updates in hardware or software configuration are avoided and regular quality checks are performed to secure validity of cross-subject and crossscan comparisons. Imaging is performed without administration of contrast agents. All imaging data are directly transferred from the scanner facility to the Erasmus MC. Data are stored on a securely backed-up research picture archiving system, using programmed scripts to check for completeness of the data received.

\section{Data management and processing}

\section{Assessment of incidental findings}

All imaging data are visually evaluated within days after acquisition by trained physicians for the presence of clinically relevant incidental findings [274]. Expert radiologists are consulted for all abnormal findings and the management of clinically relevant findings is based on protocols defined by expert panels. These protocols are updated on a regular basis incorporating the current best available knowledge regarding treatment and prognosis of the various abnormalities discovered. We are furthermore actively collaborating with ethical, legal and medical stakeholders to design a framework for the management of incidental findings that can be used both nationally and internationally for population-based imaging.

\section{Automated processing of MRI data}

Though some measurements are still performed manually or scored visually, the majority of imaging data is now processed using semi- and fully-automated computer algorithms. The Population Imaging Unit collaborates with the Biomedical Imaging Group Rotterdam (BIGR) of Erasmus MC in the application and development of automated processing pipelines for high-throughput of large data quantities. These pipelines comprise on the one end image quality checks and procedures for non-uniformity correction, normalization and image registration and on the other end advanced algorithms to extract image features to use for analyses.

Grid architectures and networked processing pipelines are used to process the large quantities or imaging data that are acquired in the Rotterdam Study.

\section{Major findings}

The Rotterdam Study research lines currently applying imaging within the Population Imaging Unit are those on neurological diseases and cardiovascular diseases.

\section{Brain imaging (MRI)}

Neurodegenerative and cerebrovascular disease are common disorders in the elderly that exert a large influence on brain functioning. Identifying underlying pathology in a preclinical state may help to recognize persons at risk, assess determinants of disease and develop preventive measures. Main objective for the Population Imaging Unit 
with respect to brain imaging is therefore to identify and quantify brain imaging biomarkers that mark the development of neurodegenerative and cerebrovascular disease.

From August 2005 onwards (RS-II-2 and onwards), brain imaging in the Population Imaging Unit is performed in all study participants without contra-indications in the context of the Rotterdam Scan Study. The current structural scanning protocol includes 4 high-resolution axial sequences (3D T1-weighted; 2D PD-weighted; 2D FLAIR; and 3D T2* GRE), 2D phase-contrast imaging, and diffusion tensor imaging (DTI). Total scanning time for these sequences amounts to approximately $30 \mathrm{~min}$. Currently, over 5,800 unique brain MRI scans and over 6,300 follow up scans have been acquired with this protocol. Starting 2012, functional imaging has been incorporated in the MRI protocol in the form of a resting-state fMRI sequence (8 min), which was added to the existing structural scans. To date, rs-fMRI scans have been acquired in over 2,000 individuals and this is ongoing.

Fully automated methods are applied to quantify atrophy of brain tissues and structures and the severity of white matter lesions [294, 469, 470]. Automated hippocampal segmentation has been successfully applied on multiscanner acquired MR images (on scans acquired in the Rotterdam Scan Study in 1995 [288] and follow up examinations in 2006), showing that a decline in hippocampal volume over a 10-year follow up period predicted onset of clinical dementia [471]. Apart from focusing only on supratentorial brain structures, we have also incorporated segmentation of the cerebellar volume into our automated processing pipelines, enabling us to study the cerebellum in aging.

Phase-contrast imaging allows for assessment of blood flow in the carotids and basilar artery. This yields measures of total brain perfusion [472], which when lower was found to be relate to worse cognition, an association that is mediated by brain atrophy [473]. More recently, we used our longitudinal imaging data to address the ongoing debate whether reduced perfusion causes brain atrophy or vice versa, and found that brain volume reduction relates to decrease in perfusion over time, presumably through lower metabolic demand [474].

The 3D T2* GRE sequence that we use was specifically developed to increase the conspicuity of cerebral microbleeds [475]. With this optimized sequence, we found that microbleed prevalence gradually increases with age, from $6.5 \%$ in persons aged 45 to 50 years to $35.7 \%$ in participants of 80 years and older; and that overall, $15.3 \%$ of all subjects over the age of 45 years has at least 1 microbleed; a much higher prevalence than was reported before [476, 477]. We found supportive evidence that deep or infratentorial microbleeds reflect arteriolosclerotic angiopathy, whereas strictly lobar microbleeds are caused by cerebral amyloid angiopathy [476, 477]. We furthermore demonstrated that incidence of microbleeds over a 3-year time interval is high and that risk factors for new microbleeds again differ according to microbleed location, in line with our findings regarding prevalent microbleeds [478]. These findings impact research into the causes of cerebral amyloid angiopathy, as well as fuel the ongoing discussion about the safety of antithrombotic therapy in persons with microbleeds [444, 479, 480]. Our most recent studies focus on the interplay between haemorrhagic and ischemic imaging markers [481], pointing towards a shared etiologic pathway. This is further supported by our finding that microbleeds relate to an increased risk of stroke, both ischemic and haemorrhagic, over a mean follow up of five years [482]. Diffusion tensor imaging (DTI) allows the assessment of the microstructural integrity of white matter. White matter microstructure loses its integrity with increasing age, but this can largely be explained by presence of white matter atrophy and white matter lesions [483]. Nevertheless, the microstructural integrity in the normal appearing white matter and in white matter lesions relates to cognitive function regardless of concurrent macrostructural changes, emphasizing the importance of the microstructural integrity of white matter [484]. Also, we found that white matter changes in normal appearing white matter are present and can be quantified on diffusion tensor imaging before white matter lesions actually develop. This suggests that white matter lesions develop gradually, and that visually appreciable lesions are only the tip of the iceberg of white matter pathology. We developed within the Rotterdam Study a method for fully automated tractography of over 20 major white matter tracts, yielding tract-specific measures of white matter integrity, enabling a more in-depth exploration of structural integrity in relation to functional processes in aging [485]. Using these tractspecific measures, we could demonstrate widespread deterioration of white matter integrity with age, which nevertheless showed variations across tract groups, with the motor system being relatively spared [486]. We are currently extending this work to assess longitudinal changes in white matter integrity and tract-specific influences on cognitive function.

\section{Imaging of atherosclerotic calcifications (MDCT)}

Main objectives with respect to imaging of vascular calcifications are to investigate distribution of and risk factors for atherosclerotic calcifications in the general elderly population and to study prognosis associated with calcifications in different vessel beds.

From September 2003 until February 2006, all participants from RS-I-4 and RS-II-2 who completed a center 
visit were invited to a MDCT scan of the coronary arteries and a second scan of the aortic arch and carotid arteries. A total of 2521 participants (response rate $79 \%$ ) were scanned. The cardiac scan reached from the apex of the heart to the tracheal bifurcation. The second scan reached from the aortic arch to the intracranial circulation. Images were analysed by trained reviewers and calcification in the different vessel beds (coronaries, aortic arch, extracranial and intracranial carotids) were quantified using the Agatston score [487].

As expected, we found that calcification load was higher overall in men compared to women, though aortic arch calcification was more prevalent among women [488]. Age and current smoking were found to be the strongest independent risk factors for arterial calcification [489]. Furthermore, strong and graded associations of prevalent stroke were found with carotid artery, aortic arch and coronary artery calcification, independent of cardiovascular risk factors [490]. When vessel calcification was studied in relation to vascular brain disease (non-invasively imaged with MRI), we found that larger intracranial carotid calcification load related to larger white matter lesion load, and that larger extracranial carotid calcification load related to the presence of cerebral infarcts, independently of ultrasound carotid plaque score. This suggests that calcification of atherosclerotic plaque yields other information in addition to merely the presence of plaques. This importance of vessel calcification is further corroborated by our recent finding that larger calcification volumes are associated with worse cognitive performance in the general population [276].

Despite extensive research on the identification of lifestyle- and environmental cardiovascular risk factors, a large part of the variability in the total burden of atherosclerosis remains yet unexplained. This inevitably indicates that other, unknown factors also considerably contribute to the development of atherosclerosis. During the last years, it has become clear that genetic factors may play an important role in the development of atherosclerosis. We determined whether previously identified genetic loci for coronary calcification were also associated with calcification in other locations than the coronary arteries Indeed, we found that the genetic basis for aortic arch and carotid artery calcification largely overlaps with that of coronary artery calcification. However, we found that the genetic variants contributed differentially to the amount of atherosclerotic calcification in these vessel beds. This suggests that also on the genetic level, differences in the etiology of atherosclerosis across vessel beds exist. Additionally, we also investigated the genetic basis of atherosclerosis using the genetics of strong risk factors of atherosclerosis, i.e. serum cholesterol levels and blood pressure and demonstrated that both serum lipids and blood pressure share a genetic predisposition with the formation of atherosclerosis in multiple vessel beds.

Worldwide, intracranial atherosclerosis is one of the leading causes of stroke, yet it is understudied in population of white descent. We therefore focused specifically on the prevalence and risk factors of intracranial internal carotid artery calcification in our population, and found that the overall prevalence of intracranial atherosclerosis was as high as $82.2 \%$. Conventional cardiovascular risk factors are associated with intracranial atherosclerosis, but risk factor profiles differed between men and women, with excessive alcohol intake and smoking were strong risk factors in men, whereas diabetes and hypertension were in women. We were the first to demonstrate in this general population-setting, that intracranial atherosclerosis is also a major cause of stroke in whites [491]. Even after adjustments for large-artery atherosclerosis in the aortic arch and the carotid artery bifurcation, this relationship was firmly present. Moreover, we found that intracranial atherosclerosis contributed to $75 \%$ of all strokes, whilst for aortic arch atherosclerosis or atherosclerosis in the carotid artery bifurcation this was 45 and $25 \%$, respectively. Altogether, these findings emphasize that existing location-specific differences in atherosclerotic burden indeed translate into differences in risk of subsequent cerebrovascular disease.

With our study on atherosclerosis and the risk of dementia, we further established the role of atherosclerosis in the etiology of dementia [276]. Most importantly, we found that systemic atherosclerosis was associated with a higher risk of dementia and cognitive decline. Actually, this suggests that generalized atherosclerosis, which probably is a better reflection of one's vascular status rather than localized atherosclerosis in a single vessel bed, associates with dementia.

\section{Carotid plaque imaging (MRI)}

Carotid wall thickening and atherosclerosis are highly prevalent at older age and are considered a major cause of cerebrovascular events [268]. Carotid atherosclerotic plaque constituents such as lipid core and haemorrhage, socalled "vulnerable" components, are considered important factors in development of clinical neurological events [492]. With MRI, it is possible to separately identify these plaque components [493]. Main objectives with respect to carotid imaging in the Rotterdam Study are to investigate distribution of and risk factors for carotid plaque components in the general elderly population and to study prognosis associated with specific carotid plaque composition.

From October 2007 onwards, all participants with carotid wall thickening of $2.5 \mathrm{~mm}$ or larger on ultrasound 
(approximately $25 \%$ of the Rotterdam Study population) are invited for carotid MRI. Imaging is performed with a bilateral phased-array surface coil (Machnet, Eelde, the Netherlands), stabilizing subjects in a custom-designed head holder to reduce motion. The imaging protocol consists of a series of high-resolution MRI sequences to image the carotid bifurcations on both sides: a PDw Fast Spin Echo (FSE) Black-blood (BB) sequence; a PDw-FSE-BB with an increased in-plane resolution; a PDw- Echo Planar Imaging (EPI) sequence and a T2w-EPI sequence; and 2 three-dimensional (3D) sequences: a 3D-T1w-Gradient Echo (GRE) sequence; and a 3D phased-contrast MRAngiography. Total scanning time amounts to approximately $30 \mathrm{~min}$.

Plaques are reviewed by trained raters for the presence of three different plaque components (calcification, haemorrhage and lipid core). Furthermore, carotid plaque size is quantified by obtaining maximum carotid wall thickness and degree of luminal stenosis using the NASCET criteria [494] on the PDw-FSE images. Postprocessing techniques aimed at automated quantification of plaque volume and identification of different plaque components are currently being developed.

So far, close to 2000 participants underwent a complete carotid MRI scan and data are currently being analysed. There is a complete overlap between carotid and brain MRI participants, allowing for the investigation of carotid plaque constituents in relation to brain imaging markers. In the first 1,006 scans, we found that intraplaque haemorrhage and lipid core were present in almost $25 \%$ of plaques, respectively, and occurred simultaneously in $9 \%$ of plaques. Different risk factors are associated with these plaque components: hypertension (and in particular high pulse pressure) and current smoking were risk factors for the presence of intraplaque haemorrhage, and hypercholesterolaemia was the only risk factor for lipid core presence.

Ischemic strokes are more often diagnosed in the left hemisphere than in the right, therefore we hypothesized that asymmetry in carotid atherosclerosis may play a role in this observation.

We indeed demonstrated that plaque prevalence, severity and composition are not equally distributed among the left and right carotid arteries [495]. Left-sided plaques were slightly thicker than the contralateral side and were predominantly composed of IPH and fibrous tissue. These findings suggest that atherosclerotic plaques on the left are more vulnerable than on the right.

Using the strength of both CT and MRI data in a subsample of our population, we were able to assess the relationship between calcification, intraplaque haemorrhage and lipid core within the carotid atherosclerotic plaque [496]. Plaques with a higher load of calcification contain more often haemorrhagic components, but less often lipid core. These findings suggest that a higher load of calcification does not imply that a plaque is more stable, and urge for prospective studies investigating the interrelation of these different plaque components with regard to future cerebrovascular events.

\section{New developments}

As also mentioned above, focus has shifted from purely structural imaging to also including functional imaging data, by incorporating resting-state functional MRI into the brain imaging protocol. Changes in the intrinsic activity of resting-state networks are presumed to represent alterations in functional brain connectivity and may mark neurodegeneration in an early, presymptomatic stage. Our initial studies will focus on the relation between functional brain connectivity and established structural markers in age-related brain diseases such as hippocampal volume, white matter lesions and microbleeds. In a later stage, we will investigate whether functional connectivity can be used an early imaging marker for dementia, by itself or in combination with other imaging markers and risk factors.

Regarding structural imaging markers, an emerging potential marker is Virchow-Robin (VR) spaces, spaces filled with interstitial fluid that surround the blood vessels in the brain and which can be dilated. Despite increasing literature on these dilated VR spaces, a major limitation of current research is the lack of a robust and generalizable rating method on MRI. Within the Rotterdam Study, we developed a novel rating method for VR spaces, which we successfully applied in 2 population-based studies, encompassing 3 different scanning protocols. We are now using this rating method to explore determinants and consequences of dilated VR spaces in our general aging population, as well as in a larger consortium of other clinical and population studies (www.uconsortium.org).

Besides ever-increasing advances in imaging hardware, software and sequence design, major advances in the short and long run are to be expected from (fully) automated image analysis. Computer processing of images will enable to make fully use of all information contained within the image, introducing new imaging biomarkers. Besides, the vast amount of imaging data that are acquired in population-based studies like the Rotterdam Study renders visual assessment or manual measurements virtually impossible, strengthening the need for (fully) automated methods of data extraction and analysis.

For additional EJE references see [13, 400, 497-500]. 


\section{Otolaryngological diseases}

\section{Objectives}

Otolaryngological research in the Rotterdam Study focuses on the frequency, etiology and consequences of auditory and vestibular disorders. We are mainly interested in dysfunctions located in the labyrinth of the inner ear, expressed by cochlear hearing loss and a deviant vestibulo-ocular reflex (VOR) for fast head movements. Etiology of both peripheral disorders will be studied, possibly revealing one or more common risk factors as both systems are connected and have similar sensory mechanisms. Additionally, we will investigate the consequences of reduced sensory input on brain development and function.

\section{Methods}

Data on both hearing and vestibular function have been collected in the cohorts RS-II-3and RS-III-2. Hearing data has also been collected in RS-I-5. Hearing loss is assessed at both ears by performing pure-tone audiometry in a sound proof room. Hearing thresholds are determined with headphones at frequencies $0.25,0.5,1,2,4$ and $8 \mathrm{kHz}$. To distinguish between cochlear and middle-ear pathology, also bone-conduction thresholds are measured at frequencies 0,5 and $4 \mathrm{kHz}$. Additionally, speech perception in noise is tested at the better ear, using a validated triplet digit test [501] with speech-shaped noise at a fixed presentation level. The ability to understand speech in noise is a functional measure that includes both sensory and central aspects of the auditory system.

Peripheral vestibular function is assessed by The Head Impulse Test (HIT), which measures the vestibule-ocular reflex (VOR) for a number of sudden head movements initiated by the tester [502]. Gain and delay are the main parameters that will be used to quantify vestibular function. An advanced infrared video-oculography system is used to track the eye movements simultaneously with the position of the head, enabling VOR recordings, even for fast head movements. Additionally, central vestibular processing is assessed by measuring the VOR during repetitive slow head movements at frequencies $0.5,1$ and $2 \mathrm{~Hz}$ while fixating the eyes at a projected visual target. The oculomotor function is tested by eye tracking of a moving target at frequencies $0.5,1$ and $2 \mathrm{~Hz}$, while the head is fixated (the smooth pursuit).

The general interview contains ten questions related to hearing and balance problems. In case of hearing-aid use, the participant has to answer five additional questions of the International Outcome Inventory of Hearing Aids (IOIHA) [503]. In case of frequent tinnitus, ten additional questions of the Short Tinnitus Handicap Inventory (THIS) are added [504].

\section{Determinants of interest}

One of our main goals is to find medical and genetic factors that are associated to age-related hearing loss. Age-related hearing loss is a common disorder that deprives older people of key sensory input. It leads to social withdrawal [505] and is even been found to be independently associated with poorer cognitive functioning and incident dementia [506, 507]. Still, little is known about the mechanisms that are responsible for developing hearing loss and the way it affects general cognitive functions within the elderly population. Determinants of interest are genetic factors, cardiovascular disease, use of medication, endocrine diseases and neuroepidemiological factors. Analyses of genetic risk factors will be performed in close collaboration with other groups within the CHARGE consortium.

Even less is known about age-related vestibular dysfunction. Problems related to balance and dizziness are frequently reported in elderly people, but there is still uncertainty about the prevalence and type of vestibular dysfunction that may cause these problems [508]. This is one of the first large cohort studies in which vestibular function will be measured. The aforementioned determinants for hearing will also be studied for the vestibular system. Additionally, the relation between hearing and peripheral vestibular function will be extensively investigated.

\section{Major findings}

In order to have sufficient statistical power and age coverage we combined the data collection of RS-II-3 and RSIII-2. First data analyses on hearing have been completed and main outcomes will be published in near future. In general, hearing loss is highly prevalent within the tested group. We found a hearing loss of $20 \mathrm{~dB} \mathrm{HL}$ or higher in more than $50 \%$ of the people. The amount of hearing loss is higher in men than in women, although the difference is smaller than previously reported and presented by the current clinical standards. As expected, hearing loss is strongly associated with age and leads to a poorer understanding of speech in background noise.

\section{Management}

The Rotterdam Study is directed by a Management Team comprising the scientific principal investigators Sarwa Darwish Murad (PI Hepatic diseases), Cornelia van Duijn (PI Genetic epidemiologic studies), Oscar Franco (PI 
Cardiovascular diseases), André Goedegebure (Otolaryngological diseases), Albert Hofman (chairman, PI Rotterdam Study), Arfan Ikram (PI Neurological diseases), Caroline Klaver (PI Ophthalmic diseases), Tamar Nijsten (PI Dermatological diseases), Robin Peeters (PI Internal Medicine), Bruno Stricker (PI Pharmaco-epidemiology), Henning Tiemeier (PI Psychiatric diseases), André Uitterlinden (PI Genomic studies), and Meike Vernooij (PI Population Imaging); and Jan Heeringa, MD, PhD, study coordinator, Eric Neeleman, head IT, and Frank van Rooij, MSc, head data-management.

\section{Emeritus principal investigators}

The following persons are Principal Investigator Emeritus of the Rotterdam Study:

Frank van den Ouweland (PI Internal Medicine 1990-1992), Diederick Grobbee (PI Cardiovascular diseases 1990-1996), Albert Hofman (PI Neurological diseases 1990-1996), Paulus de Jong (PI Ophthalmic diseases 1990-2005), Huibert Pols (PI Internal Medicine 1993-2006), Monique Breteler (PI Neurological diseases 1997-2010), Gabriel Krestin (PI Population Imaging 1998-2010), Johannes Vingerling (PI Ophthalmic diseases 2005-2010), Jacqueline Witteman (PI Cardiovascular diseases 1997-2011), Ernst Kuipers (PI Internal Medicine 2007-2013), Harry Janssen (PI Hepatic diseases 2007-2013).

Acknowledgments The Rotterdam Study is supported by the Erasmus MC University Medical Center and Erasmus University Rotterdam; The Netherlands Organisation for Scientific Research (NWO); The Netherlands Organisation for Health Research and Development (ZonMw); the Research Institute for Diseases in the Elderly (RIDE); The Netherlands Genomics Initiative (NGI); the Ministry of Education, Culture and Science; the Ministry of Health, Welfare and Sports; the European Commission (DG XII); and the Municipality of Rotterdam. The contribution of inhabitants, general practitioners and pharmacists of the Ommoord district to the Rotterdam Study is gratefully acknowledged.

Open Access This article is distributed under the terms of the Creative Commons Attribution 4.0 International License (http://crea tivecommons.org/licenses/by/4.0/), which permits unrestricted use, distribution, and reproduction in any medium, provided you give appropriate credit to the original author(s) and the source, provide a link to the Creative Commons license, and indicate if changes were made.

\section{References}

1. Oeppen J, Vaupel JW. Demography. Broken limits to life expectancy. Science. 2002;296(5570):1029-31.

2. Peto R, Doll R. There is no such thing as aging. BMJ. 1997;315(7115):1030-2.
3. Hofman A, Grobbee DE, de Jong PT, van den Ouweland FA. Determinants of disease and disability in the elderly: the Rotterdam Elderly Study. Eur J Epidemiol. 1991;7(4):403-22.

4. Hofman A, Breteler MM, van Duijn CM, Krestin GP, Pols HA, Stricker BH, et al. The Rotterdam Study: objectives and design update. Eur J Epidemiol. 2007;22(11):819-29.

5. Hofman A, van Duijn CM, Franco OH, Ikram MA, Janssen HL, Klaver CC, et al. The Rotterdam Study: 2012 objectives and design update. Eur J Epidemiol. 2011;26(8):657-86.

6. Webber L, Mytton OT, Briggs ADM, Woodcock J, Scarborough $\mathrm{P}, \mathrm{McPherson} \mathrm{K}$, et al. The Brighton declaration: the value of non-communicable disease modelling in population health sciences. Eur J Epidemiol. 2014;29(12):867-70.

7. Kruithof CJ, Kooijman MN, van Duijn CM, Franco OH, de Jongste JC, Klaver CCW, et al. The Generation R Study: biobank update 2015. Eur J Epidemiol. 2014;29(12):911-27.

8. Boffetta P, Bobak M, Borsch-Supan A, Brenner H, Eriksson S, Grodstein $\mathrm{F}$, et al. The consortium on health and ageing: network of cohorts in Europe and the United States (CHANCES) project-design, population and data harmonization of a largescale, international study. Eur J Epidemiol. 2014;29(12):929-36.

9. Zeller T, Hughes M, Tuovinen T, Schillert A, Conrads-Frank A, den Ruijter $\mathrm{H}$, et al. BiomarCaRE: rationale and design of the European BiomarCaRE project including 300,000 participants from 13 European countries. Eur J Epidemiol. 2014;29(10): 777-90.

10. Hofmann B. Diagnosing overdiagnosis: conceptual challenges and suggested solutions. Eur J Epidemiol. 2014;29(9):599-604.

11. Greiser KH, Linseisen J, Schmidt B, Wichmann HE. German Natl Cohort GNCC. The German National Cohort: aims, study design and organization. Eur J Epidemiol. 2014;29(5):371-82.

12. Karp I, Miettinen OS. On the essentials of etiological research for preventive medicine. Eur J Epidemiol. 2014;29(7):455-7.

13. Meulepas JM, Ronckers CM, Smets A, Nievelstein RAJ, Jahnen $\mathrm{A}$, Lee $\mathrm{C}$, et al. Leukemia and brain tumors among children after radiation exposure from $\mathrm{CT}$ scans: design and methodological opportunities of the Dutch Pediatric CT Study. Eur J Epidemiol. 2014;29(4):293-301.

14. Nyren O, Stenbeck M, Gronberg H. The European Parliament proposal for the new EU general data protection regulation may severely restrict European epidemiological research. Eur J Epidemiol. 2014;29(4):227-30.

15. Raoult D, Henrissat B. Are stool samples suitable for studying the link between gut microbiota and obesity? Eur J Epidemiol. 2014;29(5):307-9.

16. Rosendaal FR. National registers and their use for medical research. Eur J Epidemiol. 2014;29(8):539-40.

17. Schmidt M, Pedersen L, Sorensen HT. The Danish civil registration system as a tool in epidemiology. Eur $\mathrm{J}$ Epidemiol. 2014;29(8):541-9.

18. Thygesen LC, Ersboll AK. When the entire population is the sample: strengths and limitations in register-based epidemiology. Eur J Epidemiol. 2014;29(8):551-8.

19. Weiss NS. All-cause mortality as an outcome in epidemiologic studies: proceed with caution. Eur J Epidemiol. 2014;29(3): 147-9.

20. Forsberg JS, Hansson MG, Evers K. International guidelines on biobank research leave researchers in ambiguity: Why is this so? Eur J Epidemiol. 2013;28(6):449-51.

21. Fu M, Martinez-Sanchez JM, Sureda X, Martinez C, Ballbe M, Baranda L, et al. Handwritten versus scanned signature on the invitation letter: Does it make any difference in participation in a population-based study? Eur J Epidemiol. 2013;28(11):931-4.

22. Hunink MGM. Meta-analysis of diagnostic performance studies. Eur J Epidemiol. 2013;28(9):711-2. 
23. Jockel KH, Stang A. Cohort studies with low baseline response may not be generalisable to populations with different exposure distributions. Eur J Epidemiol. 2013;28(3):223-7.

24. Koller MT, van Delden C, Muller NJ, Baumann P, Lovis C, Marti HP, et al. Design and methodology of the Swiss Transplant Cohort Study (STCS): a comprehensive prospective nationwide long-term follow-up cohort. Eur $\mathrm{J}$ Epidemiol. 2013;28(4):347-55.

25. de Mutsert R, den Heijer M, Rabelink TJ, Smit JWA, Romijn JA, Jukema JW, et al. The Netherlands epidemiology of obesity (NEO) study: study design and data collection. Eur J Epidemiol. 2013;28(6):513-23.

26. Parker LA, Saez NG, Porta M, Hernandez-Aguado I, Lumbreras B. The impact of including different study designs in metaanalyses of diagnostic accuracy studies. Eur $\mathrm{J}$ Epidemiol. 2013;28(9):713-20.

27. VanderWeele TJ. Reconsidering the denominator of the attributable proportion for interaction. Eur J Epidemiol. 2013; 28(10):779-84.

28. Wallborn T, Grafe N, Quante M, Geserick M, Casprzig N, Ludwig J, et al. Never plan a population based cohort study on a rainy friday after a sunny thursday. Eur J Epidemiol. 2013; 28(3):285-6.

29. Dhana K, Kavousi M, Ikram MA, Tiemeier H, Hofman A, Franco OH. Body shape index in comparison with other anthropometric measures in prediction of total and causespecific mortality. J Epidemiol Community Health. 2015. doi: 10.1136/jech-2014-205257.

30. Hagg S, Fall T, Ploner A, Magi R, Fischer K, Draisma HH, et al. Adiposity as a cause of cardiovascular disease: a Mendelian randomization study. Int J Epidemiol. 2015;44(2):578-86.

31. Fall T, Hagg S, Ploner A, Magi R, Fischer K, Draisma HH, et al. Age- and sex-specific causal effects of adiposity on cardiovascular risk factors. Diabetes. 2015;64(5):1841-52.

32. Kavousi M, Leening MJ, Nanchen D, Greenland P, Graham IM, Steyerberg EW, et al. Comparison of application of the ACC/ AHA guidelines, Adult Treatment Panel III guidelines, and European Society of Cardiology guidelines for cardiovascular disease prevention in a European cohort. JAMA. 2014;311(14): 1416-23.

33. van Dijk GM, Kavousi M, Troup J, Franco OH. Health issues for menopausal women: the top 11 conditions have common solutions. Maturitas. 2015;80(1):24-30.

34. Jaspers L, Daan NM, van Dijk GM, Gazibara T, Muka T, Wen $\mathrm{KX}$, et al. Health in middle-aged and elderly women: a conceptual framework for healthy menopause. Maturitas. 2015; 81(1):93-8.

35. Leening MJ, Ferket BS, Steyerberg EW, Kavousi M, Deckers JW, Nieboer D, et al. Sex differences in lifetime risk and first manifestation of cardiovascular disease: prospective population based cohort study. BMJ. 2014;349:g5992.

36. Bleumink GS, Knetsch AM, Sturkenboom MC, Straus SM, Hofman A, Deckers JW, et al. Quantifying the heart failure epidemic: prevalence, incidence rate, lifetime risk and prognosis of heart failure the Rotterdam Study. Eur Heart J. 2004;25(18): 1614-9.

37. Heeringa J, van der Kuip DA, Hofman A, Kors JA, van Herpen $\mathrm{G}$, Stricker BH, et al. Prevalence, incidence and lifetime risk of atrial fibrillation: the Rotterdam Study. Eur Heart J. 2006;27(8): 949-53.

38. Krijthe BP, Kunst A, Benjamin EJ, Lip GY, Franco OH, Hofman A, et al. Projections on the number of individuals with atrial fibrillation in the European Union, from 2000 to 2060. Eur Heart J. 2013;34(35):2746-51.

39. Nanchen D, Leening MJ, Locatelli I, Cornuz J, Kors JA, Heeringa $\mathbf{J}$, et al. Resting heart rate and the risk of heart failure in healthy adults: the Rotterdam Study. Circ Heart Fail. 2013;6(3):403-10.

40. van Vark LC, Kardys I, Bleumink GS, Knetsch AM, Deckers JW, Hofman A, et al. Lipoprotein-associated phospholipase A2 activity and risk of heart failure: the Rotterdam Study. Eur Heart J. 2006;27(19):2346-52.

41. Heeringa J, van der Kuip DA, Hofman A, Kors JA, van Rooij FJ, Lip GY, et al. Subclinical atherosclerosis and risk of atrial fibrillation: the Rotterdam Study. Arch Intern Med. 2007;167(4): 382-7.

42. Heeringa J, Hoogendoorn EH, van der Deure WM, Hofman A, Peeters RP, Hop WC, et al. High-normal thyroid function and risk of atrial fibrillation: the Rotterdam Study. Arch Intern Med. 2008;168(20):2219-24.

43. Krijthe BP, de Jong FH, Hofman A, Franco OH, Witteman JC, Stricker $\mathrm{BH}$, et al. Dehydroepiandrosterone sulfate levels and risk of atrial fibrillation: the Rotterdam Study. Eur J Prev Cardiol. 2014;21(3):291-8.

44. Alonso A, Krijthe BP, Aspelund T, Stepas KA, Pencina MJ, Moser CB, et al. Simple risk model predicts incidence of atrial fibrillation in a racially and geographically diverse population: the CHARGE-AF consortium. J Am Heart Assoc. 2013;2(2): e000102.

45. Vasan RS, Glazer NL, Felix JF, Lieb W, Wild PS, Felix SB, et al. Genetic variants associated with cardiac structure and function: a meta-analysis and replication of genome-wide association data. JAMA. 2009;302(2):168-78.

46. Pfeufer A, van Noord C, Marciante KD, Arking DE, Larson MG, Smith AV, et al. Genome-wide association study of PR interval. Nat Genet. 2010;42(2):153-9.

47. den Hoed M, Eijgelsheim M, Esko T, Brundel BJ, Peal DS, Evans DM, et al. Identification of heart rate-associated loci and their effects on cardiac conduction and rhythm disorders. Nat Genet. 2013;45(6):621-31.

48. Benjamin EJ, Rice KM, Arking DE, Pfeufer A, van Noord C, Smith AV, et al. Variants in ZFHX3 are associated with atrial fibrillation in individuals of European ancestry. Nat Genet. 2009;41(8):879-81.

49. Ellinor PT, Lunetta KL, Albert CM, Glazer NL, Ritchie MD, Smith AV, et al. Meta-analysis identifies six new susceptibility loci for atrial fibrillation. Nat Genet. 2012;44(6):670-5.

50. Hak AE, Pols HA, Visser TJ, Drexhage HA, Hofman A, Witteman JC. Subclinical hypothyroidism is an independent risk factor for atherosclerosis and myocardial infarction in elderly women: the Rotterdam Study. Ann Intern Med. 2000;132(4):270-8.

51. Kavousi M, Elias-Smale S, Rutten JH, Leening MJ, Vliegenthart $\mathrm{R}$, Verwoert GC, et al. Evaluation of newer risk markers for coronary heart disease risk classification: a cohort study. Ann Intern Med. 2012;156(6):438-44.

52. Rutten JH, Mattace-Raso FU, Steyerberg EW, Lindemans J, Hofman A, Wieberdink RG, et al. Amino-terminal pro-B-type natriuretic peptide improves cardiovascular and cerebrovascular risk prediction in the population: the Rotterdam Study. Hypertension. 2010;55(3):785-91.

53. Kardys I, de Maat MP, Uitterlinden AG, Hofman A, Witteman JC. C-reactive protein gene haplotypes and risk of coronary heart disease: the Rotterdam Study. Eur Heart J. 2006;27(11):1331-7.

54. Oei HH, van der Meer IM, Hofman A, Koudstaal PJ, Stijnen T, Breteler MM, et al. Lipoprotein-associated phospholipase A2 activity is associated with risk of coronary heart disease and ischemic stroke: the Rotterdam Study. Circulation. 2005; 111(5):570-5.

55. van der Bom JG, de Knijff P, Haverkate F, Bots ML, Meijer P, de Jong PT, et al. Tissue plasminogen activator and risk of myocardial infarction. The Rotterdam Study. Circulation. 1997;95(12):2623-7. 
56. Ligthart S, Sedaghat S, Ikram MA, Hofman A, Franco OH, Dehghan A. EN-RAGE: a novel inflammatory marker for incident coronary heart disease. Arterioscler Thromb Vasc Biol. 2014;34(12):2695-9.

57. Koller MT, Leening MJ, Wolbers M, Steyerberg EW, Hunink MG, Schoop R, et al. Development and validation of a coronary risk prediction model for older U.S. and European persons in the Cardiovascular Health study and the Rotterdam Study. Ann Intern Med. 2012;157(6):389-97.

58. Dhana K, Ikram MA, Hofman A, Franco OH, Kavousi M. Anthropometric measures in cardiovascular disease prediction: comparison of laboratory-based versus non-laboratory-based model. Heart. 2015;101(5):377-83.

59. Bots ML, Hoes AW, Koudstaal PJ, Hofman A, Grobbee DE. Common carotid intima-media thickness and risk of stroke and myocardial infarction: the Rotterdam Study. Circulation. 1997;96(5):1432-7.

60. Mattace-Raso FU, van der Cammen TJ, Hofman A, van Popele NM, Bos ML, Schalekamp MA, et al. Arterial stiffness and risk of coronary heart disease and stroke: the Rotterdam Study. Circulation. 2006;113(5):657-63.

61. Vliegenthart R, Oudkerk M, Hofman A, Oei HH, van Dijck W, van Rooij FJ, et al. Coronary calcification improves cardiovascular risk prediction in the elderly. Circulation. 2005;112(4):572-7.

62. van der Meer IM, Bots ML, Hofman A, del Sol AI, van der Kuip DA, Witteman JC. Predictive value of noninvasive measures of atherosclerosis for incident myocardial infarction: the Rotterdam Study. Circulation. 2004;109(9):1089-94.

63. Elias-Smale SE, Proenca RV, Koller MT, Kavousi M, van Rooij FJ, Hunink MG, et al. Coronary calcium score improves classification of coronary heart disease risk in the elderly: the Rotterdam Study. J Am Coll Cardiol. 2010;56(17):1407-14.

64. Kavousi M, Leening MJ, Witteman JC. Markers for prediction of cardiovascular disease risk. JAMA. 2012;308(24):2561 author reply -2 .

65. Elias-Smale SE, Wieberdink RG, Odink AE, Hofman A, Hunink MG, Koudstaal PJ, et al. Burden of atherosclerosis improves the prediction of coronary heart disease but not cerebrovascular events: the Rotterdam Study. Eur Heart J. 2011;32(16):2050-8.

66. Leening MJ, Elias-Smale SE, Kavousi M, Felix JF, Deckers JW, Vliegenthart $\mathrm{R}$, et al. Coronary calcification and the risk of heart failure in the elderly: the Rotterdam Study. JACC Cardiovasc Imaging. 2012;5(9):874-80.

67. Kardys I, Klaver CC, Despriet DD, Bergen AA, Uitterlinden AG, Hofman A, et al. A common polymorphism in the complement factor $\mathrm{H}$ gene is associated with increased risk of myocardial infarction: the Rotterdam Study. J Am Coll Cardiol. 2006;47(8):1568-75.

68. Psaty BM, Hofman A. Genome-wide association studies and large-scale collaborations in epidemiology. Eur $\mathrm{J}$ Epidemiol. 2010;25(8):525-9.

69. Psaty BM, O’Donnell CJ, Gudnason V, Lunetta KL, Folsom AR, Rotter JI, et al. Cohorts for heart and aging research in genomic epidemiology (CHARGE) consortium: design of prospective meta-analyses of genome-wide association studies from 5 cohorts. Circ Cardiovasc Genet. 2009;2(1):73-80.

70. Dehghan A, Kottgen A, Yang Q, Hwang SJ, Kao WL, Rivadeneira F, et al. Association of three genetic loci with uric acid concentration and risk of gout: a genome-wide association study. Lancet. 2008;372(9654):1953-61.

71. Yu B, Li AH, Muzny D, Veeraraghavan N, de Vries PS, Bis JC, et al. Association of rare loss-of-function alleles in HAL, serum histidine: levels and incident coronary heart disease. Circ Cardiovasc Genet. 2015;8(2):351-5.
72. Kottgen A, Glazer NL, Dehghan A, Hwang SJ, Katz R, Li M, et al. Multiple loci associated with indices of renal function and chronic kidney disease. Nat Genet. 2009;41(6):712-7.

73. International Consortium for Blood Pressure Genome-Wide Association S, Ehret GB, Munroe PB, Rice KM, Bochud M, Johnson AD, et al. Genetic variants in novel pathways influence blood pressure and cardiovascular disease risk. Nature. 2011;478(7367):103-9.

74. Levy D, Ehret GB, Rice K, Verwoert GC, Launer LJ, Dehghan A, et al. Genome-wide association study of blood pressure and hypertension. Nat Genet. 2009;41(6):677-87.

75. Wain LV, Verwoert GC, O'Reilly PF, Shi G, Johnson T, Johnson $\mathrm{AD}$, et al. Genome-wide association study identifies six new loci influencing pulse pressure and mean arterial pressure. Nat Genet. 2011;43(10):1005-11.

76. Ganesh SK, Zakai NA, van Rooij FJ, Soranzo N, Smith AV, Nalls MA, et al. Multiple loci influence erythrocyte phenotypes in the CHARGE consortium. Nat Genet. 2009;41(11):1191-8.

77. Dehghan A, Dupuis J, Barbalic M, Bis JC, Eiriksdottir G, Lu C, et al. Meta-analysis of genome-wide association studies in $>80,000$ subjects identifies multiple loci for C-reactive protein levels. Circulation. 2011;123(7):731-8.

78. Smith JG, Platonov PG, Hedblad B, Engstrom G, Melander O. Atrial fibrillation in the Malmo diet and cancer study: a study of occurrence, risk factors and diagnostic validity. Eur J Epidemiol. 2010;25(2):95-102.

79. Huffman JE, de Vries PS, Morrison AC, Sabater-Lleal M, Kacprowski T, Auer PL, et al. Rare and low-frequency variants and their association with plasma levels of fibrinogen, FVII, FVIII, and vWF. Blood. 2015;126(11):e19-29.

80. de Vries PS, Boender J, Sonneveld MA, Rivadeneira F, Ikram MA, Rottensteiner H, et al. Genetic variants in the ADAMTS13 and SUPT3H genes are associated with ADAMTS13 activity. Blood. 2015;125(25):3949-55.

81. Bis JC, Kavousi M, Franceschini N, Isaacs A, Abecasis GR, Schminke U, et al. Meta-analysis of genome-wide association studies from the CHARGE consortium identifies common variants associated with carotid intima media thickness and plaque. Nat Genet. 2011;43(10):940-7.

82. Murabito JM, White CC, Kavousi M, Sun YV, Feitosa MF, Nambi V, et al. Association between chromosome 9p21 variants and the ankle-brachial index identified by a meta-analysis of 21 genome-wide association studies. Circ Cardiovasc Genet. 2012;5(1):100-12.

83. O'Donnell CJ, Kavousi M, Smith AV, Kardia SL, Feitosa MF, Hwang SJ, et al. Genome-wide association study for coronary artery calcification with follow-up in myocardial infarction. Circulation. 2011;124(25):2855-64.

84. Consortium CAD, Deloukas P, Kanoni S, Willenborg C, Farrall M, Assimes TL, et al. Large-scale association analysis identifies new risk loci for coronary artery disease. Nat Genet. 2013;45(1):25-33.

85. Schunkert H, Konig IR, Kathiresan S, Reilly MP, Assimes TL, Holm H, et al. Large-scale association analysis identifies 13 new susceptibility loci for coronary artery disease. Nat Genet. 2011;43(4):333-8.

86. de Vries PS, Kavousi M, Ligthart S, Uitterlinden AG, Hofman A, Franco $\mathrm{OH}$, et al. Incremental predictive value of 152 single nucleotide polymorphisms in the 10 -year risk prediction of incident coronary heart disease: the Rotterdam Study. Int J Epidemiol. 2015;44(2):682-8.

87. Steenaard RV, Ligthart S, Stolk L, Peters MJ, van Meurs JB, Uitterlinden AG, et al. Tobacco smoking is associated with methylation of genes related to coronary artery disease. Clin Epigenet. 2015;14:7. 
88. Ghanbari M, de Vries PS, de Looper H, Peters MJ, Schurmann $\mathrm{C}$, Yaghootkar $\mathrm{H}$, et al. A genetic variant in the seed region of miR-4513 shows pleiotropic effects on lipid and glucose homeostasis, blood pressure, and coronary artery disease. Hum Mutat. 2014;35(12):1524-31.

89. Ghanbari M, Sedaghat S, de Looper HW, Hofman A, Erkeland $\mathrm{SJ}$, Franco OH, et al. The association of common polymorphisms in miR-196a2 with waist to hip ratio and miR-1908 with serum lipid and glucose. Obesity (Silver Spring). 2015;23(2):495-503.

90. Ghanbari M, Franco OH, de Looper HW, Hofman A, Erkeland SJ, Dehghan A. Genetic Variations in MicroRNA-binding SITES affect MicroRNA-mediated regulation of several genes associated with cardio-metabolic phenotypes. Circ Cardiovasc Genet. 2015;8(3):473-86.

91. Heine-Broring RC, Brouwer IA, Proenca RV, van Rooij FJ, Hofman A, Oudkerk M, et al. Intake of fish and marine n-3 fatty acids in relation to coronary calcification: the Rotterdam Study. Am J Clin Nutr. 2010;91(5):1317-23.

92. van Woudenbergh GJ, Kuijsten A, Tigcheler B, Sijbrands EJ, van Rooij FJ, Hofman A, et al. Meat consumption and its association with C-reactive protein and incident type 2 diabetes: the Rotterdam Study. Diabetes Care. 2012;35(7):1499-505.

93. Muka T, Kiefte-de Jong JC, Hofman A, Dehghan A, Rivadeneira F, Franco $\mathrm{OH}$. Polyunsaturated fatty acids and serum C-reactive protein: the Rotterdam Study. Am J Epidemiol. 2015;181(11):846-56.

94. Altorf-van der Kuil W, Engberink MF, De Neve M, van Rooij FJ, Hofman A, van't Veer P, et al. Dietary amino acids and the risk of hypertension in a Dutch older population: the Rotterdam Study. Am J Clin Nutr. 2013;97(2):403-10.

95. Altorf-van der Kuil W, Engberink MF, van Rooij FJ, Hofman A, van't Veer P, Witteman JC, et al. Dietary protein and risk of hypertension in a Dutch older population: the Rotterdam Study. J Hypertens. 2010;28(12):2394-400.

96. Engberink MF, Bakker SJ, Brink EJ, van Baak MA, van Rooij FJ, Hofman A, et al. Dietary acid load and risk of hypertension: the Rotterdam Study. Am J Clin Nutr. 2012;95(6):1438-44.

97. Vitezova A, Zillikens MC, van Herpt TT, Sijbrands EJ, Hofman A, Uitterlinden AG, et al. Vitamin D status and metabolic syndrome in the elderly: the Rotterdam Study. Eur J Endocrinol. 2015;172(3):327-35.

98. Vitezova A, Cartolano NS, Heeringa J, Zillikens MC, Hofman A, Franco OH, et al. Vitamin D and the risk of atrial fibrillation-the Rotterdam Study. PLoS ONE. 2015;10(5):e0125161.

99. Mirza SS, Tiemeier H, de Bruijn RF, Hofman A, Franco OH, Kiefte-de Jong J, et al. Coffee consumption and incident dementia. Eur J Epidemiol. 2014;29(10):735-41.

100. Praagman J, Franco OH, Ikram MA, Soedamah-Muthu SS, Engberink MF, van Rooij FJ, et al. Dairy products and the risk of stroke and coronary heart disease: the Rotterdam Study. Eur J Nutr. 2015;54(6):981-90.

101. Bos D, Shahzad R, van Walsum T, van Vliet LJ, Franco OH, Hofman A, et al. Epicardial fat volume is related to atherosclerotic calcification in multiple vessel beds. Eur Heart $\mathbf{J}$ Cardiovasc Imaging. 2015

102. Jankovic N, Geelen A, Streppel MT, de Groot LC, Orfanos P, van den Hooven EH, et al. Adherence to a healthy diet according to the World Health Organization guidelines and all-cause mortality in elderly adults from Europe and the United States. Am J Epidemiol. 2014;180(10):978-88.

103. Zillikens MC, van Meurs JB, Rivadeneira F, Hofman A, Oostra BA, Sijbrands EJ, et al. Interactions between dietary vitamin E intake and SIRT1 genetic variation influence body mass index. Am J Clin Nutr. 2010;91(5):1387-93.

104. Hruby A, Ngwa JS, Renstrom F, Wojczynski MK, Ganna A, Hallmans G, et al. Higher magnesium intake is associated with lower fasting glucose and insulin, with no evidence of interaction with select genetic loci, in a meta-analysis of 15 CHARGE Consortium Studies. J Nutr. 2013;143(3):345-53.

105. Kanoni S, Nettleton JA, Hivert MF, Ye Z, van Rooij FJ, Shungin $\mathrm{D}$, et al. Total zinc intake may modify the glucose-raising effect of a zinc transporter (SLC30A8) variant: a 14-cohort metaanalysis. Diabetes. 2011;60(9):2407-16.

106. Nettleton JA, Hivert MF, Lemaitre RN, McKeown NM, Mozaffarian D, Tanaka T, et al. Meta-analysis investigating associations between healthy diet and fasting glucose and insulin levels and modification by loci associated with glucose homeostasis in data from 15 cohorts. Am J Epidemiol. 2013;177(2):103-15.

107. Dashti HS, Follis JL, Smith CE, Tanaka T, Garaulet M, Gottlieb DJ, et al. Gene-environment interactions of circadian-related genes for cardiometabolic traits. Diabetes Care. 2015;38(8):1456-66.

108. Freak-Poli R, Mirza SS, Franco OH, Ikram MA, Hofman A, Tiemeier H. Positive affect is not associated with incidence of cardiovascular disease: a population-based study of older persons. Prev Med. 2015;74:14-20.

109. Zuurbier LA, Luik AI, Leening MJ, Hofman A, Freak-Poli R, Franco $\mathrm{OH}$, et al. Associations of heart failure with sleep quality: the Rotterdam Study. J Clin Sleep Med. 2015;11(2): 117-21.

110. Leening MJ, Kavousi M, Heeringa J, van Rooij FJ, Verkroostvan Heemst J, Deckers JW, et al. Methods of data collection and definitions of cardiac outcomes in the Rotterdam Study. Eur J Epidemiol. 2012;27(3):173-85.

111. Smith NL, Chen MH, Dehghan A, Strachan DP, Basu S, Soranzo $\mathrm{N}$, et al. Novel associations of multiple genetic loci with plasma levels of factor VII, factor VIII, and von Willebrand factor: The CHARGE (Cohorts for heart and aging research in genome epidemiology) consortium. Circulation. 2010;121(12):1382-92.

112. Dehghan A, Kardys I, de Maat MP, Uitterlinden AG, Sijbrands EJ, Bootsma $\mathrm{AH}$, et al. Genetic variation, C-reactive protein levels, and incidence of diabetes. Diabetes. 2007;56(3):872-8.

113. Straus SM, Kors JA, De Bruin ML, van der Hooft CS, Hofman A, Heeringa J, et al. Prolonged QTc interval and risk of sudden cardiac death in a population of older adults. J Am Coll Cardiol. 2006;47(2):362-7.

114. Kardys I, Deckers JW, Stricker BH, Vletter WB, Hofman A, Witteman J. Distribution of echocardiographic parameters and their associations with cardiovascular risk factors in the Rotterdam Study. Eur J Epidemiol. 2010;25(7):481-90.

115. Klipstein-Grobusch K, den Breeijen JH, Goldbohm RA, Geleijnse JM, Hofman A, Grobbee DE, et al. Dietary assessment in the elderly: validation of a semiquantitative food frequency questionnaire. Eur J Clin Nutr. 1998;52(8):588-96.

116. Feunekes GI, Van Staveren WA, De Vries JH, Burema J, Hautvast JG. Relative and biomarker-based validity of a foodfrequency questionnaire estimating intake of fats and cholesterol. Am J Clin Nutr. 1993;58(4):489-96.

117. Goldbohm RA, van den Brandt PA, Brants HA, van't Veer P, Al $\mathrm{M}$, Sturmans $\mathrm{F}$, et al. Validation of a dietary questionnaire used in a large-scale prospective cohort study on diet and cancer. Eur J Clin Nutr. 1994;48(4):253-65.

118. Goldbohm RA, van't Veer P, van den Brandt PA, van't Hof MA, van't Hof HA, Brants HA, Sturmans F, et al. Reproducibility of a food frequency questionnaire and stability of dietary habits determined from five annually repeated measurements. Eur J Clin Nutr. 1995;49(6):420-9.

119. Siebelink E, Geelen A, de Vries JH. Self-reported energy intake by FFQ compared with actual energy intake to maintain body weight in 516 adults. Br J Nutr. 2011;106(2):274-81.

120. Caspersen CJ, Bloemberg BP, Saris WH, Merritt RK, Kromhout D. The prevalence of selected physical activities and their 
relation with coronary heart disease risk factors in elderly men: the Zutphen Study, 1985. Am J Epidemiol. 1991;133(11): 1078-92.

121. Stel VS, Smit JH, Pluijm SM, Visser M, Deeg DJ, Lips P. Comparison of the LASA Physical Activity Questionnaire with a 7-day diary and pedometer. J Clin Epidemiol. 2004;57(3):252-8.

122. Voorrips LE, Ravelli AC, Dongelmans PC, Deurenberg P, Van Staveren WA. A Physical Activity Questionnaire for the elderly. Med Sci Sports Exerc. 1991;23(8):974-9.

123. Arabshahi S, Busingye D, Subasinghe AK, Evans RG, Riddell MA, Thrift AG. Adiposity has a greater impact on hypertension in lean than not-lean populations: a systematic review and metaanalysis. Eur J Epidemiol. 2014;29(5):311-24.

124. Blix K, Braekkan SK, le Cessie S, Skjeldestad FE, Cannegieter SC, Hansen JB. The increased risk of venous thromboembolism by advancing age cannot be attributed to the higher incidence of cancer in the elderly: the Tromso study. Eur J Epidemiol. 2014;29(4):277-84.

125. Eveborn GW, Schirmer H, Lunde P, Heggelund G, Hansen JB, Rasmussen K. Assessment of risk factors for developing incident aortic stenosis: the Tromso study. Eur J Epidemiol. 2014;29(8):567-75.

126. Greenwood DC, Thatcher NJ, Ye J, Garrard L, Keogh G, King LG, et al. Caffeine intake during pregnancy and adverse birth outcomes: a systematic review and dose-response meta-analysis. Eur J Epidemiol. 2014;29(10):725-34.

127. Gunnell AS, Knuiman MW, Divitini ML, Cormie P. Leisure time physical activity and long-term cardiovascular and cancer outcomes: the Busselton Health study. Eur J Epidemiol. 2014;29(11):851-7.

128. Hillesund ER, Overby NC, Engel SM, Klungsoyr K, Harmon $\mathrm{QE}$, Haugen M, et al. Associations of adherence to the New Nordic Diet with risk of preeclampsia and preterm delivery in the Norwegian Mother and Child Cohort Study (MoBa). Eur J Epidemiol. 2014;29(10): 753-65.

129. Holtermann A, Mork PJ, Nilsen TIL. Hours lying down per day and mortality from all-causes and cardiovascular disease: the HUNT Study, Norway. Eur J Epidemiol. 2014;29(8):559-65.

130. Horvei LD, Braekkan SK, Mathiesen EB, Njolstad I, Wilsgaard T, Hansen JB. Obesity measures and risk of venous thromboembolism and myocardial infarction. Eur $\mathrm{J}$ Epidemiol. 2014;29(11):821-30.

131. Icks A, Claessen H, Kirchberger I, Heier M, Peters A, Trentinaglia I, et al. Mortality after first myocardial infarction in diabetic and non-diabetic people between 1985 and 2009. The MONICA/ KORA registry. Eur J Epidemiol. 2014;29(12):899-909.

132. Ilar A, Lewne M, Plato N, Hallqvist J, Alderling M, Bigert C, et al. Myocardial infarction and occupational exposure to motor exhaust: a population-based case-control study in Sweden. Eur J Epidemiol. 2014;29(7):517-25.

133. James JE. Personalised medicine, disease prevention, and the inverse care law: More harm than benefit? Eur J Epidemiol. 2014;29(6):383-90.

134. Jdanov DA, Deev AD, Jasilionis D, Shalnova SA, Shkolnikova MA, Shkolnikov VM. Recalibration of the SCORE risk chart for the Russian population. Eur J Epidemiol. 2014;29(9):621-8.

135. Knuiman M, Briffa T, Divitini M, Chew D, Eikelboom J, McQuillan B, et al. A cohort study examination of established and emerging risk factors for atrial fibrillation: the Busselton Health study. Eur J Epidemiol. 2014;29(3):181-90.

136. Leenders M, Boshuizen HC, Ferrari P, Siersema PD, Overvad K, Tjonneland $\mathrm{A}$, et al. Fruit and vegetable intake and causespecific mortality in the EPIC study. Eur J Epidemiol. 2014;29(9):639-52.

137. Martinsson A, Andersson C, Andell P, Koul S, Engstrom G, Smith JG. Anemia in the general population: prevalence, clinical correlates and prognostic impact. Eur $\mathrm{J}$ Epidemiol. 2014;29(7):489-98.

138. Niedziela J, Hudzik B, Niedziela N, Gasior M, Gierlotka M, Wasilewski J, et al. The obesity paradox in acute coronary syndrome: a meta-analysis. Eur $\mathrm{J}$ Epidemiol. 2014;29(11):801-12.

139. Nordahl H, Rod NH, Frederiksen BL, Andersen I, Lange T, Diderichsen F, et al. Education and risk of coronary heart disease: assessment of mediation by behavioral risk factors using the additive hazards model. Eur J Epidemiol. 2013;28(2):149-57.

140. O'Doherty MG, Jorgensen T, Borglykke A, Brenner H, Schottker B, Wilsgaard T, et al. Repeated measures of body mass index and C-reactive protein in relation to all-cause mortality and cardiovascular disease: results from the consortium on health and ageing network of cohorts in Europe and the United States (CHANCES). Eur J Epidemiol. 2014;29(12):887-97.

141. Song X, Pukkala E, Dyba T, Tuomilehto J, Moltchanov V, Mannisto $S$, et al. Body mass index and cancer incidence: the FINRISK study. Eur J Epidemiol. 2014;29(7):477-87.

142. Turney BW, Appleby PN, Reynard JM, Noble JG, Key TJ, Allen NE. Diet and risk of kidney stones in the Oxford cohort of the European prospective investigation into cancer and nutrition (EPIC). Eur J Epidemiol. 2014;29(5):363-9.

143. Ueda P, Cnattingius S, Stephansson O, Ingelsson E, Ludvigsson JF, Bonamy AKE. Cerebrovascular and ischemic heart disease in young adults born preterm: a population-based Swedish cohort study. Eur J Epidemiol. 2014;29(4):253-60.

144. Wald NJ, Morris JK. Quantifying the health benefits of chronic disease prevention: a fresh approach using cardiovascular disease as an example. Eur J Epidemiol. 2014;29(9):605-12.

145. Ahmadi-Abhari S, Luben RN, Wareham NJ, Khaw KT. Seventeen year risk of all-cause and cause-specific mortality associated with C-reactive protein, fibrinogen and leukocyte count in men and women: the EPIC-Norfolk study. Eur J Epidemiol. 2013;28(7):541-50.

146. Etemadi A, Abnet CC, Kamangar F, Islami F, Khademi H, Pourshams A, et al. Impact of body size and physical activity during adolescence and adult life on overall and cause-specific mortality in a large cohort study from Iran. Eur J Epidemiol. 2014;29(2):95-109.

147. Gellert C, Schottker B, Muller H, Holleczek B, Brenner H. Impact of smoking and quitting on cardiovascular outcomes and risk advancement periods among older adults. Eur J Epidemiol. 2013;28(8):649-58.

148. Gram IT, Sandin S, Braaten T, Lund E, Weiderpass E. The hazards of death by smoking in middle-aged women. Eur $\mathrm{J}$ Epidemiol. 2013;28(10):799-806.

149. His M, Zelek L, Deschasaux M, Pouchieu C, Kesse-Guyot E, Hercberg S, et al. Prospective associations between serum biomarkers of lipid metabolism and overall, breast and prostate cancer risk. Eur J Epidemiol. 2014;29(2):119-32.

150. Iversen B, Jacobsen BK, Lochen ML. Active and passive smoking and the risk of myocardial infarction in 24,968 men and women during 11 year of follow-up: the Tromso study. Eur $\mathbf{J}$ Epidemiol. 2013;28(8):659-67.

151. Jha P. The 21 st century benefits of smoking cessation in Europe. Eur J Epidemiol. 2013;28(8):617-9.

152. Julin B, Wolk A, Thomas LD, Akesson A. Exposure to cadmium from food and risk of cardiovascular disease in men: a population-based prospective cohort study. Eur J Epidemiol. 2013;28(10):837-40.

153. Kelly-Irving M, Lepage B, Dedieu D, Bartley M, Blane D, Grosclaude $\mathrm{P}$, et al. Adverse childhood experiences and premature allcause mortality. Eur J Epidemiol. 2013;28(9):721-34.

154. Kulik MC, Hoffmann R, Judge K, Looman C, Menvielle G, Kulhanova I, et al. Smoking and the potential for reduction of 
inequalities in mortality in Europe. Eur $\mathrm{J}$ Epidemiol. 2013;28(12):959-71.

155. Kunutsor SK, Burgess S, Munroe PB, Khan H. Vitamin D and high blood pressure: Causal association or epiphenomenon? Eur J Epidemiol. 2014;29(1):1-14.

156. Li KR, Monni S, Husing A, Wendt A, Kneisel J, Gross ML, et al. Primary preventive potential of major lifestyle risk factors for acute myocardial infarction in men: an analysis of the EPICHeidelberg cohort. Eur J Epidemiol. 2014;29(1):27-34.

157. Malerba S, Turati F, Galeone C, Pelucchi C, Verga F, La Vecchia C, et al. A meta-analysis of prospective studies of coffee consumption and mortality for all causes, cancers and cardiovascular diseases. Eur J Epidemiol. 2013;28(7):527-39.

158. Marshall RJ. Predicting cardiovascular events. Eur J Epidemiol. 2014;29(2):145.

159. Novak M, Toren K, Lappas G, Kok WG, Jern C, Wilhelmsen L, et al. Occupational status and incidences of ischemic and hemorrhagic stroke in Swedish men: a population-based 35-year prospective follow-up study. Eur J Epidemiol. 2013;28(8):697-704.

160. Schmidt M, Botker HE, Pedersen L, Sorensen HT. Adult height and risk of ischemic heart disease, atrial fibrillation, stroke, venous thromboembolism, and premature death: a population based 36-year follow-up study. Eur J Epidemiol. 2014;29(2): $111-8$.

161. Ueda P, Bonamy AKE, Granath F, Cnattingius S. Month of birth and cause-specific mortality between 50 and 80 years: a population-based longitudinal cohort study in Sweden. Eur J Epidemiol. 2014;29(2):89-94.

162. Bamia C, Lagiou P, Buckland G, Grioni S, Agnoli C, Taylor AJ, et al. Mediterranean diet and colorectal cancer risk: results from a European cohort. Eur J Epidemiol. 2013;28(4):317-28.

163. Behrens G, Fischer B, Kohler S, Park Y, Hollenbeck AR, Leitzmann MF. Healthy lifestyle behaviors and decreased risk of mortality in a large prospective study of U.S. women and men. Eur J Epidemiol. 2013;28(5):361-72.

164. Brown MC, Best KE, Pearce MS, Waugh J, Robson SC, Bell R. Cardiovascular disease risk in women with pre-eclampsia: systematic review and meta-analysis. Eur J Epidemiol. 2013; 28(1):1-19.

165. Clays E, De Bacquer D, Janssens H, De Clercq B, Casini A, Braeckman $\mathrm{L}$, et al. The association between leisure time physical activity and coronary heart disease among men with different physical work demands: a prospective cohort study. Eur J Epidemiol. 2013;28(3):241-7.

166. Faeh D, Rohrmann S, Braun J. Better risk assessment with glycated hemoglobin instead of cholesterol in CVD risk prediction charts. Eur J Epidemiol. 2013;28(7):551-5.

167. Hjellvik V, Selmer R, Gjessing HK, Tverdal A, Vollset SE. Body mass index, smoking, and risk of death between 40 and 70 years of age in a Norwegian cohort of 32,727 women and 33,475 men. Eur J Epidemiol. 2013;28(1):35-43.

168. Jorde R, Schirmer H, Njolstad I, Lochen ML, Mathiesen EB, Kamycheva E, et al. Serum calcium and the calcium-sensing receptor polymorphism rs 17251221 in relation to coronary heart disease, type 2 diabetes, cancer and mortality: the Tromso study. Eur J Epidemiol. 2013;28(7):569-78.

169. Karppi J, Kurl S, Makikallio TH, Ronkainen K, Laukkanen JA. Low levels of plasma carotenoids are associated with an increased risk of atrial fibrillation. Eur $\mathrm{J}$ Epidemiol. 2013;28(1):45-53.

170. Kunutsor SK, Apekey TA, Steur M. Vitamin D and risk of future hypertension: meta-analysis of 283,537 participants. Eur J Epidemiol. 2013;28(3):205-21.

171. Leening MJG, Cook NR. Net reclassification improvement: a link between statistics and clinical practice. Eur J Epidemiol. 2013;28(1):21-3.
172. Leon DA, Shkolnikov VM, Borinskaya S, Casas JP, Evans A, Gil A, et al. Hazardous alcohol consumption is associated with increased levels of B-type natriuretic peptide: evidence from two population-based studies. Eur J Epidemiol. 2013;28(5):393-404.

173. Mackenbach JP. Convergence and divergence of life expectancy in Europe: a centennial view. Eur J Epidemiol. 2013;28(3): 229-40.

174. McKee M, Legido-Quigley H, Piot P. Trends in life expectancy in Europe: one big explanation or many small ones? Eur $\mathrm{J}$ Epidemiol. 2013;28(3):203-4.

175. Miettola S, Hartikainen AL, Vaarasmaki M, Bloigu A, Ruokonen A, Jarvelin MR, et al. Offspring's blood pressure and metabolic phenotype after exposure to gestational hypertension in utero. Eur J Epidemiol. 2013;28(1):87-98.

176. Mishra GD, Chiesa F, Goodman A, De Stavola B, Koupil I. Socio-economic position over the life course and all-cause, and circulatory diseases mortality at age $50-87$ years: results from a Swedish birth cohort. Eur J Epidemiol. 2013;28(2):139-47.

177. Muhlenbruch K, Heraclides A, Steyerberg EW, Joost HG, Boeing $\mathrm{H}$, Schulze MB. Assessing improvement in disease prediction using net reclassification improvement: impact of risk cut-offs and number of risk categories. Eur $\mathrm{J}$ Epidemiol. 2013;28(1):25-33.

178. Panczak R, Galobardes B, Spoerri A, Zwahlen M, Egger M. High life in the sky? Mortality by floor of residence in Switzerland. Eur J Epidemiol. 2013;28(6):453-62.

179. Ribeiro A, Fatima de Pina M. Could moderate earthquakes also trigger cardiac events? Eur J Epidemiol. 2013;28(2):199-202.

180. Takahara M, Katakami N, Kaneto H, Shimomura I. Risk categorization for calculating net reclassification improvement. Eur J Epidemiol. 2013;28(7):607-9.

181. Threapleton DE, Greenwood DC, Burley VJ, Aldwairji M, Cade JE. Dietary fibre and cardiovascular disease mortality in the UK Women's Cohort study. Eur J Epidemiol. 2013;28(4):335-46.

182. Wu YL, Li SR, Zhang DF. Circulating 25-hydroxyvitamin D levels and hypertension risk. Eur J Epidemiol. 2013;28(7): 611-6.

183. Flohil SC, van der Leest RJ, Dowlatshahi EA, Hofman A, de Vries E, Nijsten T. Prevalence of actinic keratosis and its risk factors in the general population: the Rotterdam Study. J Invest Dermatol. 2013;133(8):1971-8.

184. Verkouteren JA, Pardo LM, Uitterlinden AG, Hofman A, Nijsten T. Common variants affecting susceptibility to develop multiple basal cell carcinomas. J Invest Dermatol. 2015;135(8):2135-8.

185. Jacobs LC, Hamer MA, Gunn DA, Deelen J, Lall JS, van Heemst $\mathrm{D}$, et al. A genome-wide association study identifies the skin color genes IRF4, MC1R, ASIP, and BNC2 influencing facial pigmented spots. J Invest Dermatol. 2015;135(7):1735-42.

186. Jacobs LC, Wollstein A, Lao O, Hofman A, Klaver CC, Uitterlinden $\mathrm{AG}$, et al. Comprehensive candidate gene study highlights UGT1A and BNC2 as new genes determining continuous skin color variation in Europeans. Hum Genet. 2013;132(2):147-58.

187. Liu F, Visser M, Duffy DL, Hysi PG, Jacobs LC, Lao O, et al. Genetics of skin color variation in Europeans: genome-wide association studies with functional follow-up. Hum Genet. 2015;134(8):823-35.

188. Dowlatshahi EA, Kavousi M, Nijsten T, Ikram MA, Hofman A, Franco $\mathrm{OH}$, et al. Psoriasis is not associated with atherosclerosis and incident cardiovascular events: the Rotterdam Study. J Invest Dermatol. 2013;133(10):2347-54.

189. van der Voort EA, Koehler EM, Dowlatshahi EA, Hofman A, Stricker BH, Janssen HL, et al. Psoriasis is independently associated with nonalcoholic fatty liver disease in patients 55 years old or older: results from a population-based study. J Am Acad Dermatol. 2014;70(3):517-24. 
190. van der Voort EA, Koehler EM, Nijsten T, Stricker BH, Hofman A, Janssen HL, et al. Increased prevalence of advanced liver fibrosis in patients with psoriasis: a cross-sectional analysis from the Rotterdam Study. Acta Derm Venereol. 2015. doi:10.2340/ 00015555-2161.

191. Oei L, Rivadeneira F, Ly F, Breda SJ, Zillikens MC, Hofman A, et al. Review of radiological scoring methods of osteoporotic vertebral fractures for clinical and research settings. Eur Radiol. 2013;23(2):476-86.

192. Makurthou AA, Oei L, Saddy SE, Breda SJ, Castano-Betancourt MC, Hofman A, et al. Scheuermann's disease: evaluation of radiological criteria and population prevalence. Spine (Phila Pa 1976). 2013.

193. Oei L, Zillikens MC, Dehghan A, Buitendijk GH, Castano-Betancourt MC, Estrada K, et al. High bone mineral density and fracture risk in type 2 diabetes as skeletal complications of inadequate glucose control: the Rotterdam Study. Diabetes Care. 2013;36(6):1619-28.

194. Castano-Betancourt MC, Rivadeneira F, Bierma-Zeinstra S, Kerkhof HJ, Hofman A, Uitterlinden AG, et al. Bone parameters across different types of hip osteoarthritis and their relationship to osteoporotic fracture risk. Arthritis Rheum. 2013;65(3):693-700.

195. Castano-Betancourt MC, Van Meurs JB, Bierma-Zeinstra S, Rivadeneira F, Hofman A, Weinans H, et al. The contribution of hip geometry to the prediction of hip osteoarthritis. Osteoarthritis Cartilage. 2013;21(10):1530-6.

196. Kerkhof HJ, Bierma-Zeinstra SM, Arden NK, Metrustry S, Castano-Betancourt M, Hart DJ, et al. Prediction model for knee osteoarthritis incidence, including clinical, genetic and biochemical risk factors. Ann Rheum Dis. 2014;73(12):2116-21.

197. Valdes AM, Meulenbelt I, Chassaing E, Arden NK, BiermaZeinstra S, Hart D, et al. Large scale meta-analysis of urinary C-terminal telopeptide, serum cartilage oligomeric protein and matrix metalloprotease degraded type II collagen and their role in prevalence, incidence and progression of osteoarthritis. Osteoarthritis Cartilage. 2014;22(5):683-9.

198. Hoeven TA, Kavousi M, Clockaerts S, Kerkhof HJ, van Meurs JB, Franco O, et al. Association of atherosclerosis with presence and progression of osteoarthritis: the Rotterdam Study. Ann Rheum Dis. 2013;72(5):646-51.

199. Hoeven TA, Leening MJ, Bindels PJ, Castano-Betancourt M, van Meurs JB, Franco OH, et al. Disability and not osteoarthritis predicts cardiovascular disease: a prospective population-based cohort study. Ann Rheum Dis. 2015;74(4):752-6.

200. Schiphof D, Kerkhof HJ, Damen J, de Klerk BM, Hofman A, Koes $\mathrm{BW}$, et al. Factors for pain in patients with different grades of knee osteoarthritis. Arthritis Care Res (Hoboken). 2013;65(5):695-702.

201. de Kruijf M, Kerkhof HJ, Peters MJ, Bierma-Zeinstra S, Hofman A, Uitterlinden AG, et al. Finger length pattern as a biomarker for osteoarthritis and chronic joint pain: a populationbased study and meta-analysis after systematic review. Arthritis Care Res (Hoboken). 2014;66(9):1337-43.

202. Chaker L, Buitendijk GH, Dehghan A, Medici M, Hofman A, Vingerling JR, et al. Thyroid function and age-related macular degeneration: a prospective population-based cohort study-the Rotterdam Study. BMC Med. 2015;13:94.

203. Medici M, Direk N, Visser WE, Korevaar TI, Hofman A, Visser TJ, et al. Thyroid function within the normal range and the risk of depression: a population-based cohort study. J Clin Endocrinol Metab. 2014;99(4):1213-9.

204. van der Loos MJ, Haring R, Rietveld CA, Baumeister SE, Groenen PJ, Hofman A, et al. Serum testosterone levels in males are not associated with entrepreneurial behavior in two independent observational studies. Physiol Behav. 2013;2(119):110-4.

205. Blum MR, Bauer DC, Collet TH, Fink HA, Cappola AR, da Costa BR, et al. Subclinical thyroid dysfunction and fracture risk: a meta-analysis. JAMA. 2015;313(20):2055-65.
206. Chaker L, Baumgartner C, den Elzen WP, Ikram MA, Blum MR, Collet TH, et al. Subclinical hypothyroidism and the risk of stroke events and fatal stroke: an individual participant data analysis. J Clin Endocrinol Metab. 2015;100(6):2181-91.

207. Asvold BO, Vatten LJ, Bjoro T, Bauer DC, Bremner A, Cappola $\mathrm{AR}$, et al. Thyroid function within the normal range and risk of coronary heart disease: an individual participant data analysis of 14 cohorts. JAMA Intern Med. 2015;175(6):1037-47.

208. Estrada K, Styrkarsdottir U, Evangelou E, Hsu YH, Duncan EL, Ntzani EE, et al. Genome-wide meta-analysis identifies 56 bone mineral density loci and reveals 14 loci associated with risk of fracture. Nat Genet. 2012;44(5):491-501.

209. Zeggini E, Panoutsopoulou K, Southam L, Rayner NW, et al. Identification of new susceptibility loci for osteoarthritis (arcOGEN): a genome-wide association study. Lancet. 2012;380(9844):815-823.

210. Castano Betancourt MC, Cailotto F, Kerkhof HJ, Cornelis FM, Doherty SA, Hart DJ, et al. Genome-wide association and functional studies identify the DOT1L gene to be involved in cartilage thickness and hip osteoarthritis. Proc Natl Acad Sci USA. 2012;109(21):8218-23.

211. Peters MJ, Broer L, Willemen HL, Eiriksdottir G, Hocking LJ, Holliday KL, et al. Genome-wide association study meta-analysis of chronic widespread pain: evidence for involvement of the 5p15.2 region. Ann Rheum Dis. 2013;72(3):427-36.

212. Rodriguez-Fontenla C, Calaza M, Evangelou E, Valdes AM, Arden N, Blanco FJ, et al. Assessment of osteoarthritis candidate genes in a meta-analysis of nine genome-wide association studies. Arthritis Rheumatol. 2014;66(4):940-9.

213. Ramos YF, Metrustry S, Arden N, Bay-Jensen AC, Beekman M, de Craen AJ, et al. Meta-analysis identifies loci affecting levels of the potential osteoarthritis biomarkers SCOMP and uCTX-II with genome wide significance. J Med Genet. 2014;51(9): 596-604.

214. Styrkarsdottir U, Thorleifsson G, Helgadottir HT, Bomer N, Metrustry S, Bierma-Zeinstra S, et al. Severe osteoarthritis of the hand associates with common variants within the ALDH1A2 gene and with rare variants at 1p31. Nat Genet. 2014;46(5):498-502.

215. Stolk L, Perry JR, Chasman DI, He C, Mangino M, Sulem P, et al. Meta-analyses identify 13 loci associated with age at menopause and highlight DNA repair and immune pathways. Nat Genet. 2012;44(3):260-8.

216. Porcu E, Medici M, Pistis G, Volpato CB, Wilson SG, Cappola $\mathrm{AR}$, et al. A meta-analysis of thyroid-related traits reveals novel loci and gender-specific differences in the regulation of thyroid function. PLoS Genet. 2013;9(2):e1003266.

217. Medici M, Porcu E, Pistis G, Teumer A, Brown SJ, Jensen RA, et al. Identification of novel genetic loci associated with thyroid peroxidase antibodies and clinical thyroid disease. PLoS Genet. 2014;10(2):e1004123.

218. Schultheiss UT, Teumer A, Medici M, Li Y, Daya N, Chaker L, et al. A genetic risk score for thyroid peroxidase antibodies associates with clinical thyroid disease in community-based populations. J Clin Endocrinol Metab. 2015;100(5):E799-807.

219. Mayerle J, den Hoed CM, Schurmann C, Stolk L, Homuth G, Peters MJ, et al. Identification of genetic loci associated with Helicobacter pylori serologic status. JAMA. 2013;309(18):1912-20.

220. Aune D, Norat T, Romundstad P, Vatten LJ. Whole grain and refined grain consumption and the risk of type 2 diabetes: a systematic review and dose-response meta-analysis of cohort studies. Eur J Epidemiol. 2013;28(11):845-58.

221. Bloch F. Not only the traumatic falls in elderly subjects requires awareness but all the falls even those hitherto regarded as unimportant. Eur J Epidemiol. 2014;29(9):663-4.

222. Buijsse B, Boeing H, Hirche F, Weikert C, Schulze MB, Gottschald $\mathrm{M}$, et al. Plasma 25-hydroxyvitamin D and its genetic 
determinants in relation to incident type 2 diabetes: a prospective case-cohort study. Eur J Epidemiol. 2013;28(9):743-52.

223. Chaker L, Baumgartner C, Ikram MA, Dehghan A, Medici M, Visser WE, et al. Subclinical thyroid dysfunction and the risk of stroke: a systematic review and meta-analysis. Eur J Epidemiol. 2014;29(11):791-800.

224. Dai XY, Yuan J, Yao P, Yang BY, Gui LX, Zhang XM, et al. Association between serum uric acid and the metabolic syndrome among a middle- and old-age Chinese population. Eur $\mathbf{J}$ Epidemiol. 2013;28(8):669-76.

225. Fagherazzi G, Vilier A, Lajous M, Boutron-Ruault MC, Balkau B, Clavel-Chapelon F, et al. Wine consumption throughout life is inversely associated with type 2 diabetes risk, but only in overweight individuals: results from a large female French cohort study. Eur J Epidemiol. 2014;29(11):831-9.

226. Frisch M, Nielsen NM, Pedersen BV. Same-sex marriage, autoimmune thyroid gland dysfunction and other autoimmune diseases in Denmark 1989-2008. Eur J Epidemiol. 2014;29(1): 63-71.

227. Janghorbani M, Momeni F, Mansourian M. Systematic review and metaanalysis of air pollution exposure and risk of diabetes. Eur J Epidemiol. 2014;29(4):231-42.

228. Lagiou P, Samoli E, Hsieh CC, Lagiou A, Xu B, Yu GP, et al. Maternal and cord blood hormones in relation to birth size. Eur $\mathrm{J}$ Epidemiol. 2014;29(5):343-51.

229. Lahousse L, Maes B, Ziere G, Loth DW, Verlinden VJA, Zillikens MC, et al. Adverse outcomes of frailty in the elderly: the Rotterdam Study. Eur J Epidemiol. 2014;29(6):419-27.

230. Min JY, Min KB. Serum C-peptide levels as an independent predictor of diabetes mellitus mortality in non-diabetic individuals. Eur J Epidemiol. 2013;28(9):771-4.

231. Naver KV, Secher NJ, Ovesen PG, Gorst-Rasmussen A, Lundbye-Christensen S, Nilas L. Offspring preterm birth and birth size are related to long-term risk of maternal diabetes. Eur $\mathbf{J}$ Epidemiol. 2013;28(5):427-32.

232. Ostroumova E, Gudzenko N, Brenner A, Gorokh Y, Hatch M, Prysyazhnyuk A, et al. Thyroid cancer incidence in Chornobyl liquidators in Ukraine: SIR analysis, 1986-2010. Eur J Epidemiol. 2014;29(5):337-42.

233. Sabanayagam C, Wong TY, Xiao J, Shankar A. Serum cystatin $\mathrm{C}$ and prediabetes in non-obese US adults. Eur $\mathrm{J}$ Epidemiol. 2013;28(4):311-6.

234. Satman I, Omer B, Tutuncu Y, Kalaca S, Gedik S, Dinccag N, et al. Twelve-year trends in the prevalence and risk factors of diabetes and prediabetes in Turkish adults. Eur $\mathbf{J}$ Epidemiol. 2013;28(2):169-80.

235. Saum KU, Dieffenbach AK, Muller H, Holleczek B, Hauer K, Brenner H. Frailty prevalence and 10-year survival in community-dwelling older adults: results from the ESTHER cohort study. Eur J Epidemiol. 2014;29(3):171-9.

236. Schmid D, Behrens G, Jochem C, Keimling M, Leitzmann M. Physical activity, diabetes, and risk of thyroid cancer: a systematic review and meta-analysis. Eur $\mathrm{J}$ Epidemiol. 2013;28(12):945-58.

237. Schottker B, Herder C, Rothenbacher D, Perna L, Muller H, Brenner H. Serum 25-hydroxyvitamin D levels and incident diabetes mellitus type 2: a competing risk analysis in a large population-based cohort of older adults. Eur $\mathrm{J}$ Epidemiol. 2013;28(3):267-75

238. Schottker B, Saum KU, Perna L, Ordonez-Mena JM, Holleczek $\mathrm{B}$, Brenner $\mathrm{H}$. Is vitamin D deficiency a cause of increased morbidity and mortality at older age or simply an indicator of poor health? Eur J Epidemiol. 2014;29(3):199-210.

239. Schram MT, Sep SJS, van der Kallen CJ, Dagnelie PC, Koster A, Schaper N, et al. The Maastricht Study: an extensive phenotyping study on determinants of type 2 diabetes, its complications and its comorbidities. Eur $\mathrm{J}$ Epidemiol. 2014;29(6):439-51.

240. Vinceti M, Malagoli C, Rothman KJ, Rodolfi R, Astolfi G, Calzolari E, et al. Risk of birth defects associated with maternal pregestational diabetes. Eur J Epidemiol. 2014;29(6):411-8.

241. Weyer V, Blettner M, Cholmakow-Bodechtel C, Heudorf U. Chemical accident at Hoechst AG Frankfurt/Main, Germany, 1993: a 15 year follow-up analysis of mortality. Eur J Epidemiol. 2014;29(1):73-6.

242. Yao BD, Fang H, Xu WH, Yan YJ, Xu HL, Liu YN, et al. Dietary fiber intake and risk of type 2 diabetes: a dose-response analysis of prospective studies. Eur J Epidemiol. 2014;29(2): 79-88.

243. Hamaguchi M, Kojima T, Itoh Y, Harano Y, Fujii K, Nakajima $\mathrm{T}$, et al. The severity of ultrasonographic findings in nonalcoholic fatty liver disease reflects the metabolic syndrome and visceral fat accumulation. Am J Gastroenterol. 2007;102(12):2708-15.

244. Sandrin L, Fourquet B, Hasquenoph JM, Yon S, Fournier C, Mal F, et al. Transient elastography: a new noninvasive method for assessment of hepatic fibrosis. Ultrasound Med Biol. 2003; 29(12):1705-13.

245. Talwalkar JA, Kurtz DM, Schoenleber SJ, West CP, Montori VM. Ultrasound-based transient elastography for the detection of hepatic fibrosis: systematic review and meta-analysis. Clin Gastroenterol Hepatol. 2007;5(10):1214-20.

246. Koehler EM, Schouten JN, Hansen BE, van Rooij FJ, Hofman A, Stricker BH, et al. Prevalence and risk factors of non-alcoholic fatty liver disease in the elderly: results from the Rotterdam Study. J Hepatol. 2012;57(6):1305-11.

247. de Keyser CE, Koehler EM, Schouten JN, Visser LE, Hofman A, Janssen HL, et al. Statin therapy is associated with a reduced risk of non-alcoholic fatty liver in overweight individuals. Dig Liver Dis. 2014;46(8):720-5.

248. Koehler EM, Schouten JN, Hansen BE, Hofman A, Stricker BH, Janssen HL. External validation of the fatty liver index for identifying nonalcoholic fatty liver disease in a populationbased study. Clin Gastroenterol Hepatol. 2013;11(9):1201-4.

249. Koehler EM, Sanna D, Hansen BE, van Rooij FJ, Heeringa J, Hofman A, et al. Serum liver enzymes are associated with allcause mortality in an elderly population. Liver Int. 2014;34(2): 296-304.

250. Plompen EP, Hansen BE, Schouten JN, Darwish Murad S, Loth DW, Brouwer WP, et al. Interferon gamma receptor 2 gene variants are associated with liver fibrosis in the general population: the Rotterdam Study. Gut. 2015;64(4):692-4.

251. Ott A, Breteler MM, van Harskamp F, Claus JJ, van der Cammen TJ, Grobbee DE, et al. Prevalence of Alzheimer's disease and vascular dementia: association with education. The Rotterdam Study. BMJ. 1995;310(6985):970-3.

252. de Rijk MC, Breteler MM, Graveland GA, Ott A, Grobbee DE, van der Meche FG, et al. Prevalence of Parkinson's disease in the elderly: the Rotterdam Study. Neurology. 1995;45(12):2143-6.

253. Ruitenberg A, Ott A, van Swieten JC, Hofman A, Breteler MM. Incidence of dementia: Does gender make a difference? Neurobiol Aging. 2001;22(4):575-80.

254. Hollander M, Koudstaal PJ, Bots ML, Grobbee DE, Hofman A, Breteler MM. Incidence, risk, and case fatality of first ever stroke in the elderly population. The Rotterdam Study. J Neurol Neurosurg Psychiatry. 2003;74(3):317-21.

255. de Lau LM, Giesbergen PC, de Rijk MC, Hofman A, Koudstaal PJ, Breteler MM. Incidence of parkinsonism and Parkinson disease in a general population: the Rotterdam Study. Neurology. 2004;63(7):1240-4.

256. Ikram MA, Wieberdink RG, Koudstaal PJ. International epidemiology of intracerebral hemorrhage. Curr Atheroscler Rep. 2012;14(4):300-6. 
257. Schrijvers EM, Verhaaren BF, Koudstaal PJ, Hofman A, Ikram MA, Breteler MM. Is dementia incidence declining? Trends in dementia incidence since 1990 in the Rotterdam Study. Neurology. 2012;78(19):1456-63.

258. Wieberdink RG, Ikram MA, Hofman A, Koudstaal PJ, Breteler MM. Trends in stroke incidence rates and stroke risk factors in Rotterdam, the Netherlands from 1990 to 2008. Eur J Epidemiol. 2012;27(4):287-95.

259. Breteler MM, Claus JJ, Grobbee DE, Hofman A. Cardiovascular disease and distribution of cognitive function in elderly people: the Rotterdam Study. BMJ. 1994;308(6944):1604-8.

260. de Bruijn RF, Ikram MA. Cardiovascular risk factors and future risk of Alzheimer's disease. BMC Med. 2014;12:130.

261. Hofman A, Ott A, Breteler MM, Bots ML, Slooter AJ, van Harskamp F, et al. Atherosclerosis, apolipoprotein E, and prevalence of dementia and Alzheimer's disease in the Rotterdam Study. Lancet. 1997;349(9046):151-4.

262. Ott A, Slooter AJ, Hofman A, van Harskamp F, Witteman JC, Van Broeckhoven C, et al. Smoking and risk of dementia and Alzheimer's disease in a population-based cohort study: the Rotterdam Study. Lancet. 1998;351(9119):1840-3.

263. Ruitenberg A, van Swieten JC, Witteman JC, Mehta KM, van Duijn CM, Hofman A, et al. Alcohol consumption and risk of dementia: the Rotterdam Study. Lancet. 2002;359(9303):281-6.

264. de Bruijn RF, Schrijvers EM, de Groot KA, Witteman JC, Hofman A, Franco OH, et al. The association between physical activity and dementia in an elderly population: the Rotterdam Study. Eur J Epidemiol. 2013;28(3):277-83.

265. in t' Veld BA, Ruitenberg A, Hofman A, Launer LJ, van Duijn $\mathrm{CM}$, Stijnen T, et al. Nonsteroidal antiinflammatory drugs and the risk of Alzheimer's disease. N Engl J Med. 2001;345(21): 1515-21.

266. Euser SM, van Bemmel T, Schram MT, Gussekloo J, Hofman A, Westendorp RG, et al. The effect of age on the association between blood pressure and cognitive function later in life. J Am Geriatr Soc. 2009;57(7):1232-7.

267. Euser SM, Sattar N, Witteman JC, Bollen EL, Sijbrands EJ, Hofman A, et al. A prospective analysis of elevated fasting glucose levels and cognitive function in older people: results from PROSPER and the Rotterdam Study. Diabetes. 2010;59(7):1601-7.

268. Hollander M, Bots ML, Del Sol AI, Koudstaal PJ, Witteman JC, Grobbee DE, et al. Carotid plaques increase the risk of stroke and subtypes of cerebral infarction in asymptomatic elderly: the Rotterdam Study. Circulation. 2002;105(24):2872-7.

269. Wieberdink RG, Koudstaal PJ, Hofman A, Witteman JC, Breteler MM, Ikram MA. Insulin resistance and the risk of stroke and stroke subtypes in the nondiabetic elderly. Am J Epidemiol. 2012;176(8):699-707.

270. Wieberdink RG, Poels MM, Vernooij MW, Koudstaal PJ, Hofman A, van der Lugt A, et al. Serum lipid levels and the risk of intracerebral hemorrhage: the Rotterdam Study. Arterioscler Thromb Vasc Biol. 2011;31(12):2982-9.

271. Bos MJ, Schipper CM, Koudstaal PJ, Witteman JC, Hofman A, Breteler MM. High serum C-reactive protein level is not an independent predictor for stroke: the Rotterdam Study. Circulation. 2006;114(15):1591-8.

272. Ferket BS, van Kempen BJ, Wieberdink RG, Steyerberg EW, Koudstaal PJ, Hofman A, et al. Separate prediction of intracerebral hemorrhage and ischemic stroke. Neurology. 2014; 82(20):1804-12.

273. Vermeer SE, Prins ND, den Heijer T, Hofman A, Koudstaal PJ, Breteler MM. Silent brain infarcts and the risk of dementia and cognitive decline. N Engl J Med. 2003;348(13):1215-22.

274. Vernooij MW, Ikram MA, Tanghe HL, Vincent AJ, Hofman A, Krestin GP, et al. Incidental findings on brain MRI in the general population. N Engl J Med. 2007;357(18):1821-8.
275. de Bruijn RF, Portegies ML, Leening MJ, Bos MJ, Hofman A, van der Lugt A, et al. Subclinical cardiac dysfunction increases the risk of stroke and dementia: the Rotterdam Study. Neurology. 2015;84(8):833-40.

276. Bos D, Vernooij MW, de Bruijn RF, Koudstaal PJ, Hofman A, Franco $\mathrm{OH}$, et al. Atherosclerotic calcification is related to a higher risk of dementia and cognitive decline. Alzheimers Dement. 2015;11(6):639-647 e1.

277. Sajjad A, Mirza SS, Portegies ML, Bos MJ, Hofman A, Koudstaal PJ, et al. Subjective memory complaints and the risk of stroke. Stroke. 2015;46(1):170-5.

278. Desikan RS, Schork AJ, Wang Y, Thompson WK, Dehghan A, Ridker $\mathrm{PM}$, et al. Polygenic overlap between C-reactive protein, plasma lipids, and Alzheimer disease. Circulation. 2015;131(23):2061-9.

279. Ikram MA, Seshadri S, Bis JC, Fornage M, DeStefano AL, Aulchenko YS, et al. Genomewide association studies of stroke. N Engl J Med. 2009;360(17):1718-28.

280. Lambert JC, Ibrahim-Verbaas CA, Harold D, Naj AC, Sims R, Bellenguez C, et al. Meta-analysis of 74,046 individuals identifies 11 new susceptibility loci for Alzheimer's disease. Nat Genet. 2013;45(12):1452-8.

281. Schrijvers EM, Schurmann B, Koudstaal PJ, van den Bussche H, Van Duijn CM, Hentschel F, et al. Genome-wide association study of vascular dementia. Stroke. 2012;43(2):315-9.

282. Seshadri S, Fitzpatrick AL, Ikram MA, DeStefano AL, Gudnason $\mathrm{V}$, Boada M, et al. Genome-wide analysis of genetic loci associated with Alzheimer disease. JAMA. 2010;303(18):1832-40.

283. Verhaaren BF, Vernooij MW, Dehghan A, Vrooman HA, de Boer R, Hofman A, et al. The relation of uric acid to brain atrophy and cognition: the Rotterdam Scan Study. Neuroepidemiology. 2013;41(1):29-34.

284. Breteler MM, van Swieten JC, Bots ML, Grobbee DE, Claus JJ, van den Hout JH, et al. Cerebral white matter lesions, vascular risk factors, and cognitive function in a population-based study: the Rotterdam Study. Neurology. 1994;44(7):1246-52.

285. Ikram MA, van der Lugt A, Niessen WJ, Krestin GP, Koudstaal PJ, Hofman A, et al. The Rotterdam Scan Study: design and update up to 2012. Eur J Epidemiol. 2011;26(10):811-24.

286. Ikram MA, van Oijen M, de Jong FJ, Kors JA, Koudstaal PJ, Hofman A, et al. Unrecognized myocardial infarction in relation to risk of dementia and cerebral small vessel disease. Stroke. 2008;39(5):1421-6.

287. Ikram MA, Vernooij MW, Hofman A, Niessen WJ, van der Lugt A, Breteler MM. Kidney function is related to cerebral small vessel disease. Stroke. 2008;39(1):55-61.

288. Ikram MA, Vrooman HA, Vernooij MW, van der Lijn F, Hofman A, van der Lugt A, et al. Brain tissue volumes in the general elderly population. The Rotterdam Scan Study. Neurobiol Aging. 2008;29(6):882-90.

289. Ikram MK, de Jong FJ, Vernooij MW, Hofman A, Niessen WJ, van der Lugt A, et al. Retinal vascular calibers associate differentially with cerebral gray matter and white matter atrophy. Alzheimer Dis Assoc Disord. 2013;27(4):351-5.

290. Poels MM, Zaccai K, Verwoert GC, Vernooij MW, Hofman A, van der Lugt A, et al. Arterial stiffness and cerebral small vessel disease: the Rotterdam Scan Study. Stroke. 2012;43(10):2637-42.

291. Poels MM, Steyerberg EW, Wieberdink RG, Hofman A, Koudstaal PJ, Ikram MA, et al. Assessment of cerebral small vessel disease predicts individual stroke risk. J Neurol Neurosurg Psychiatry. 2012;83(12):1174-9.

292. Saavedra Perez HC, Direk N, Hofman A, Vernooij MW, Tiemeier H, Ikram MA. Silent brain infarcts: A cause of depression in the elderly? Psychiatry Res. 2013;211(2):180-2.

293. Ikram MA, Vernooij MW, Vrooman HA, Hofman A, Breteler MM. Brain tissue volumes and small vessel disease in relation to the risk of mortality. Neurobiol Aging. 2009;30(3):450-6. 
294. Ikram MA, Vrooman HA, Vernooij MW, den Heijer T, Hofman A, Niessen WJ, et al. Brain tissue volumes in relation to cognitive function and risk of dementia. Neurobiol Aging. 2010;31(3):378-86.

295. Verlinden VJ, van der Geest JN, de Groot M, Hofman A, Niessen WJ, van der Lugt A, et al. Structural and microstructural brain changes predict impairment in daily functioning. Am J Med. 2014;127(11):1089-1096 e2.

296. Bis JC, DeCarli C, Smith AV, van der Lijn F, Crivello F, Fornage $\mathrm{M}$, et al. Common variants at $12 \mathrm{q} 14$ and $12 \mathrm{q} 24$ are associated with hippocampal volume. Nat Genet. 2012;44(5): 545-51.

297. Debette S, Bis JC, Fornage M, Schmidt H, Ikram MA, Sigurdsson S, et al. Genome-wide association studies of MRI-defined brain infarcts: meta-analysis from the CHARGE consortium. Stroke. 2010;41(2):210-7.

298. Fornage M, Debette S, Bis JC, Schmidt H, Ikram MA, Dufouil $\mathrm{C}$, et al. Genome-wide association studies of cerebral white matter lesion burden: the CHARGE consortium. Ann Neurol. 2011;69(6):928-39.

299. Ikram MA, DeCarli C. Next frontiers in the genetic epidemiology of Alzheimer's disease. Eur J Epidemiol. 2012;27(11): 831-6.

300. Ikram MA, Fornage M, Smith AV, Seshadri S, Schmidt R, Debette S, et al. Common variants at $6 \mathrm{q} 22$ and $17 \mathrm{q} 21$ are associated with intracranial volume. Nat Genet. 2012;44(5): 539-44.

301. Stein JL, Medland SE, Vasquez AA, Hibar DP, Senstad RE, Winkler AM, et al. Identification of common variants associated with human hippocampal and intracranial volumes. Nat Genet. 2012;44(5):552-61.

302. Verhaaren BF, de Boer R, Vernooij MW, Rivadeneira F, Uitterlinden AG, Hofman A, et al. Replication study of chr17q25 with cerebral white matter lesion volume. Stroke. 2011;42(11): 3297-9.

303. Verlinden VJ, van der Geest JN, Hoogendam YY, Hofman A, Breteler MM, Ikram MA. Gait patterns in a community-dwelling population aged 50 years and older. Gait Posture. 2013;37(4): $500-5$.

304. Loehrer E, Vernooij MW, van der Lugt A, Hofman A, Ikram MA. Migraine and cerebral blood flow in the general population. Cephalalgia. 2015;35(2):190-8.

305. Bos MJ, van Rijn MJ, Witteman JC, Hofman A, Koudstaal PJ, Breteler MM. Incidence and prognosis of transient neurological attacks. JAMA. 2007;298(24):2877-85.

306. Hoogendam YY, Hofman A, van der Geest JN, van der Lugt A, Ikram MA. Patterns of cognitive function in aging: the Rotterdam Study. Eur J Epidemiol. 2014;29(2):133-40.

307. Killgore WD, Glahn DC, Casasanto DJ. Development and validation of the design organization test (DOT): a rapid screening instrument for assessing visuospatial ability. J Clin Exp Neuropsychol. 2005;27(4):449-59.

308. Trouillas P, Takayanagi T, Hallett M, Currier RD, Subramony $\mathrm{SH}$, Wessel K, et al. International Cooperative Ataxia Rating Scale for pharmacological assessment of the cerebellar syndrome. The Ataxia Neuropharmacology Committee of the World Federation of Neurology. J Neurol Sci. 1997;145(2): 205-11.

309. de Bruijn R, Schrijvers EMC, de Groot KA, Witteman JCM, Hofman A, Franco OH, et al. The association between physical activity and dementia in an elderly population: the Rotterdam Study (vol 28, pg 277, 2013). Eur J Epidemiol. 2013;28(5):447-8.

310. Dufouil C, Pereira E, Chene G, Glymour MM, Alperovitch A, Saubusse E, et al. Older age at retirement is associated with decreased risk of dementia. Eur $\mathrm{J}$ Epidemiol. 2014;29(5):353-61.
311. Feldman AL, Wirdefeldt K, Johansson ALV, Gatz M, Pedersen NL. Evidence for modest familial co-aggregation between dementia and parkinsonism. Eur J Epidemiol. 2014;29(1):49-56.

312. Grant WB. The role of milk protein in increasing risk of Parkinson's disease. Eur J Epidemiol. 2013;28(4):357.

313. Hamidou B, Couratier P, Besancon C, Nicol M, Preux PM, Marin B. Epidemiological evidence that physical activity is not a risk factor for ALS. Eur J Epidemiol. 2014;29(7):459-75.

314. Hoogendam YY, Hofman A, van der Geest JN, van der Lugt A, Ikram MA. Patterns of cognitive function in aging: the Rotterdam Study. Eur J Epidemiol. 2014;29(2):133-40.

315. Jacqmin-Gadda H, Alperovitch A, Montlahuc C, Commenges D, Leffondre K, Dufouil C, et al. 20-year prevalence projections for dementia and impact of preventive policy about risk factors. Eur J Epidemiol. 2013;28(6):493-502.

316. Jiang WJ, Ju CX, Jiang H, Zhang DF. Dairy foods intake and risk of Parkinson's disease: a dose-response meta-analysis of prospective cohort studies. Eur J Epidemiol. 2014;29(9):613-9.

317. Kyrozis A, Ghika A, Stathopoulos P, Vassilopoulos D, Trichopoulos D, Trichopoulou A. Dietary and lifestyle variables in relation to incidence of Parkinson's disease in Greece. Eur J Epidemiol. 2013;28(1):67-77.

318. Lai SW, Liao KF, Lin CL, Sung FC. Irritable bowel syndrome correlates with increased risk of Parkinson's disease in Taiwan. Eur J Epidemiol. 2014;29(1):57-62.

319. Marioni RE, Proust-Lima C, Amieva H, Brayne C, Matthews FE, Dartigues JF, et al. Cognitive lifestyle jointly predicts longitudinal cognitive decline and mortality risk. Eur J Epidemiol. 2014;29(3):211-9.

320. Mensikova K, Kanovsky P, Kaiserova M, Mikulicova L, Vastik M, Hlustik $P$, et al. Prevalence of neurodegenerative parkinsonism in an isolated population in south-eastern Moravia, Czech Republic. Eur J Epidemiol. 2013;28(10):833-6.

321. Mons U, Schottker B, Muller H, Kliegel M, Brenner H. History of lifetime smoking, smoking cessation and cognitive function in the elderly population. Eur J Epidemiol. 2013;28(10):823-31.

322. Nevalainen O, Raitanen J, Ansakorpi H, Artama M, Isojarvi J, Auvinen A. Long-term mortality risk by cause of death in newly diagnosed patients with epilepsy in Finland: a nationwide register-based study. Eur J Epidemiol. 2013;28(12):981-90.

323. Palm F, Dos Santos M, Urbanek C, Greulich M, Zimmer K, Safer A, et al. Stroke seasonality associations with subtype, etiology and laboratory results in the Ludwigshafen Stroke study (LuSSt). Eur J Epidemiol. 2013;28(5):373-81.

324. Pekmezovic T, Jovic J, Svetel M, Kostic VS. Prevalence of restless legs syndrome among adult population in a Serbian district: a community-based study. Eur J Epidemiol. 2013; 28(11):927-30.

325. Pellegrino P, Carnovale C, Perrone V, Antoniazzi S, Pozzi M, Clementi E, et al. No evidence of a link between multiple sclerosis and the vaccine against the human papillomavirus. Eur J Epidemiol. 2013;28(8):705-7.

326. Schernhammer E, Qiu JH, Wermuth L, Lassen CF, Friis S, Ritz B. Gout and the risk of Parkinson's disease in Denmark. Eur J Epidemiol. 2013;28(4):359-60.

327. Skoog I. Is it dangerous or beneficial to drink coffee? Reflections on a meta-analysis on risk at birth and a population study on risk in late life. Eur J Epidemiol. 2014;29(10):665-6.

328. Virta JJ, Heikkila K, Perola M, Koskenvuo M, Raiha I, Rinne $\mathrm{JO}$, et al. Midlife cardiovascular risk factors and late cognitive impairment. Eur J Epidemiol. 2013;28(5):405-16.

329. Zhang ZZ, Xu GL, Liu DZ, Zhu WS, Fan XY, Liu XF. Dietary fiber consumption and risk of stroke. Eur J Epidemiol. 2013;28(2):119-30.

330. Klein R, Myers CE, Buitendijk GH, Rochtchina E, Gao X, de Jong PT, et al. Lipids, lipid genes, and incident age-related 
macular degeneration: the three continent age-related macular degeneration consortium. Am J Ophthalmol. 2014;158(3): 513-524 e3.

331. Wang JJ, Buitendijk GH, Rochtchina E, Lee KE, Klein BE, van Duijn CM, et al. Genetic susceptibility, dietary antioxidants, and long-term incidence of age-related macular degeneration in two populations. Ophthalmology. 2014;121(3):667-75.

332. Buitendijk GH, Amin N, Hofman A, van Duijn CM, Vingerling JR, Klaver CC. Direct-to-consumer personal genome testing for age-related macular degeneration. Invest Ophthalmol Vis Sci. 2014;55(10):6167-74.

333. Williams KM, Verhoeven VJ, Cumberland P, Bertelsen G, Wolfram C, Buitendijk GH, et al. Prevalence of refractive error in Europe: the European eye epidemiology (E(3)) consortium. Eur J Epidemiol. 2015;30(4):305-15.

334. Williams KM, Bertelsen G, Cumberland P, Wolfram C, Verhoeven VJ, Anastasopoulos E, et al. Increasing prevalence of myopia in Europe and the impact of education. Ophthalmology. 2015;122(7):1489-97.

335. Verhoeven VJ, Wong KT, Buitendijk GH, Hofman A, Vingerling JR, Klaver CC. Visual consequences of refractive errors in the general population. Ophthalmology. 2015;122(1):101-9.

336. Cheng CY, Schache M, Ikram MK, Young TL, Guggenheim JA, Vitart $\mathrm{V}$, et al. Nine loci for ocular axial length identified through genome-wide association studies, including shared loci with refractive error. Am J Hum Genet. 2013;93(2):264-77.

337. Simpson CL, Wojciechowski R, Oexle K, Murgia F, Portas L, Li $\mathrm{X}$, et al. Genome-wide meta-analysis of myopia and hyperopia provides evidence for replication of 11 loci. PLOS ONE. 2014;9(9):e107110.

338. Li Q, Wojciechowski R, Simpson CL, Hysi PG, Verhoeven VJ, Ikram MK, et al. Genome-wide association study for refractive astigmatism reveals genetic co-determination with spherical equivalent refractive error: the CREAM consortium. Hum Genet. 2015;134(2):131-46.

339. Hysi PG, Cheng CY, Springelkamp H, Macgregor S, Bailey JN, Wojciechowski R, et al. Genome-wide analysis of multiancestry cohorts identifies new loci influencing intraocular pressure and susceptibility to glaucoma. Nat Genet. 2014; 46(10):1126-30.

340. Springelkamp H, Iglesias AI, Cuellar-Partida G, Amin N, Burdon KP, van Leeuwen EM, et al. ARHGEF12 influences the risk of glaucoma by increasing intraocular pressure. Hum Mol Genet. 2015;24(9):2689-99.

341. Springelkamp H, Hohn R, Mishra A, Hysi PG, Khor CC, Loomis SJ, et al. Meta-analysis of genome-wide association studies identifies novel loci that influence cupping and the glaucomatous process. Nat Commun. 2014;5:4883.

342. Springelkamp H, Mishra A, Hysi PG, Gharahkhani P, Hohn R, Khor CC, et al. Meta-analysis of genome-wide association studies identifies novel loci associated with optic disc morphology. Genet Epidemiol. 2015;39(3):207-16.

343. Iglesias AI, Springelkamp H, van der Linde H, Severijnen LA, Amin N, Oostra B, et al. Exome sequencing and functional analyses suggest that SIX6 is a gene involved in an altered proliferation-differentiation balance early in life and optic nerve degeneration at old age. Hum Mol Genet. 2014;23(5):1320-32.

344. Springelkamp H, Lee K, Wolfs RC, Buitendijk GH, Ramdas WD, Hofman A, et al. Population-based evaluation of retinal nerve fiber layer, retinal ganglion cell layer, and inner plexiform layer as a diagnostic tool for glaucoma. Invest Ophthalmol Vis Sci. 2014;55(12):8428-38.

345. He R, Shen J, Zhao J, Zeng H, Li LX, Zhao JG, et al. High cystatin $\mathrm{C}$ levels predict severe retinopathy in type 2 diabetes patients. Eur J Epidemiol. 2013;28(9):775-8.
346. La Torre G, Pacella E, Saulle R, Giraldi G, Pacella F, Lenzi T, et al. The synergistic effect of exposure to alcohol, tobacco smoke and other risk factors for age-related macular degeneration. Eur J Epidemiol. 2013;28(5):445-6.

347. Lai SW, Lin CL, Liao KF. Cataract may be a non-memory feature of Alzheimer's disease in older people. Eur J Epidemiol. 2014;29(6):405-9.

348. Verhoeven VJM, Buitendijk GHS, Rivadeneira F, Uitterlinden AG, Vingerling JR, Hofman A, et al. Education influences the role of genetics in myopia. Eur J Epidemiol. 2013;28(12):973-80.

349. Wang YX, Xu L, Jonas JB. The effect of the Chinese cultural revolution and great leap forward on the prevalence of myopia. Eur J Epidemiol. 2013;28(12):1001-4.

350. Tiemeier H, van Dijck W, Hofman A, Witteman JC, Stijnen T, Breteler MM. Relationship between atherosclerosis and late-life depression: the Rotterdam Study. Arch Gen Psychiatry. 2004;61(4):369-76.

351. Hek K, Demirkan A, Lahti J, Terracciano A, Teumer A, Cornelis MC, et al. A genome-wide association study of depressive symptoms. Biol Psychiatry. 2013;73(7):667-78.

352. Gottlieb DJ, Hek K, Chen TH, Watson NF, Eiriksdottir G, Byrne EM, et al. Novel loci associated with usual sleep duration: the CHARGE consortium genome-wide association study. Mol Psychiatry. 2014. doi:10.1038/mp.2014.133.

353. Bolton JL, Hayward C, Direk N, Lewis JG, Hammond GL, Hill LA, et al. Genome wide association identifies common variants at the SERPINA6/SERPINA1 locus influencing plasma cortisol and corticosteroid binding globulin. PLoS Genet. 2014;10(7): e1004474.

354. Ganna A, Rivadeneira F, Hofman A, Uitterlinden AG, Magnusson PK, Pedersen NL, et al. Genetic determinants of mortality. Can findings from genome-wide association studies explain variation in human mortality? Hum Genet. 2013;132(5): 553-61.

355. Krijthe BP, Walter S, Newson RS, Hofman A, Hunink MG, Tiemeier H. Is positive affect associated with survival? A population-based study of elderly persons. Am J Epidemiol. 2011;173(11):1298-307.

356. Luijendijk HJ, van den Berg JF, Dekker MJ, van Tuijl HR, Otte $\mathrm{W}$, Smit F, et al. Incidence and recurrence of late-life depression. Arch Gen Psychiatry. 2008;65(12):1394-401.

357. Hek K, Direk N, Newson RS, Hofman A, Hoogendijk WJ, Mulder CL, et al. Anxiety disorders and salivary cortisol levels in older adults: a population-based study. Psychoneuroendocrinology. 2013;38(2):300-5.

358. Van Den Berg JF, Van Rooij FJ, Vos H, Tulen JH, Hofman A, Miedema HM, et al. Disagreement between subjective and actigraphic measures of sleep duration in a population-based study of elderly persons. J Sleep Res. 2008;17(3):295-302.

359. Luik AI, Zuurbier LA, Whitmore H, Hofman A, Tiemeier H. REM sleep and depressive symptoms in a population-based study of middle-aged and elderly persons. J Sleep Res. 2015;24(3):305-8.

360. Zuurbier LA, Luik AI, Hofman A, Franco OH, Van Someren EJ, Tiemeier H. Fragmentation and stability of circadian activity rhythms predict mortality: the Rotterdam Study. Am J Epidemiol. 2015;181(1):54-63.

361. Newson RS, Hek K, Luijendijk HJ, Hofman A, Witteman JC, Tiemeier H. Atherosclerosis and incident depression in late life. Arch Gen Psychiatry. 2010;67(11):1144-51.

362. Direk N, Koudstaal PJ, Hofman A, Ikram MA, Hoogendijk WJ, Tiemeier H. Cerebral hemodynamics and incident depression: the Rotterdam Study. Biol Psychiatry. 2012;72(4):318-23.

363. van den Berg JF, Miedema HM, Tulen JH, Hofman A, Neven $\mathrm{AK}$, Tiemeier H. Sex differences in subjective and actigraphic 
sleep measures: a population-based study of elderly persons. Sleep. 2009;32(10):1367-75.

364. Luik AI, Noteboom J, Zuurbier LA, Whitmore H, Hofman A, Tiemeier H. Sleep apnea severity and depressive symptoms in a population-based study. Sleep Health. 2015;1:128-32.

365. Hek K, Tiemeier H, Newson RS, Luijendijk HJ, Hofman A, Mulder CL. Anxiety disorders and comorbid depression in community dwelling older adults. Int J Methods Psychiatr Res. 2011;20(3):157-68

366. Newson RS, Boelen PA, Hek K, Hofman A, Tiemeier H. The prevalence and characteristics of complicated grief in older adults. J Affect Disord. 2011;132(1-2):231-8.

367. Saavedra Perez HC, Ikram MA, Direk N, Prigerson HG, FreakPoli R, Verhaaren BF, et al. Cognition, structural brain changes and complicated grief. A population-based study. Psychol Med. 2015;45(7):1389-99.

368. Rietveld CA, Cesarini D, Benjamin DJ, Koellinger PD, De Neve JE, Tiemeier $\mathrm{H}$, et al. Molecular genetics and subjective wellbeing. Proc Natl Acad Sci USA. 2013;110(24):9692-7.

369. Velders FP, Kuningas M, Kumari M, Dekker MJ, Uitterlinden AG, Kirschbaum C, et al. Genetics of cortisol secretion and depressive symptoms: a candidate gene and genome wide association approach. Psychoneuroendocrinology. 2011;36(7): 1053-61.

370. Walter S, Mackenbach J, Voko Z, Lhachimi S, Ikram MA, Uitterlinden AG, et al. Genetic, physiological, and lifestyle predictors of mortality in the general population. Am J Public Health. 2012;102(4):e3-10.

371. Anic GM, Madden MH, Sincich K, Thompson RC, Nabors LB, Olson JJ, et al. Early life exposures and the risk of adult glioma. Eur J Epidemiol. 2013;28(9):753-8.

372. Binachon B, Dossus L, Danjou AMN, Clavel-Chapelon F, Fervers B. Life in urban areas and breast cancer risk in the French E3N cohort. Eur J Epidemiol. 2014;29(10):743-51.

373. Eliasen M, Becker U, Gronbaek M, Juel K, Tolstrup JS. Alcoholattributable and alcohol-preventable mortality in Denmark: an analysis of which intake levels contribute most to alcohol's harmful and beneficial effects. Eur J Epidemiol. 2014;29(1):15-26.

374. Hwang IC, Shin D. Urinary sodium excretion is associated with short sleep duration. Eur J Epidemiol. 2014;29(12):937-40.

375. Kershaw KN, Droomers M, Robinson WR, Carnethon MR, Daviglus ML, Verschuren WMM. Quantifying the contributions of behavioral and biological risk factors to socioeconomic disparities in coronary heart disease incidence: the MORGEN study. Eur J Epidemiol. 2013;28(10):807-14.

376. Koppes LLJ, Geuskens GA, Pronk A, Vermeulen RCH, de Vroome EMM. Night work and breast cancer risk in a general population prospective cohort study in the Netherlands. Eur J Epidemiol. 2014;29(8):577-84.

377. Leinonen T, Lahelma E, Martikainen P. Trajectories of antidepressant medication before and after retirement: the contribution of socio-demographic factors. Eur J Epidemiol. 2013;28(5): 417-26.

378. Liu C, Urquia M, Cnattingius S, Hjern A. Migration and preterm birth in war refugees: a Swedish cohort study. Eur J Epidemiol. 2014;29(2):141-3.

379. Luque-Fernandez MA, Franco M, Gelaye B, Schomaker M, Garitano IG, D'Este C, et al. Unemployment and stillbirth risk among foreign-born and Spanish pregnant women in Spain, 2007-2010: a multilevel analysis study. Eur J Epidemiol. 2013;28(12):991-9.

380. Pequignot R, Tzourio C, Peres K, Ancellin ML, Perier MC, Ducimetiere $\mathrm{P}$, et al. Depressive symptoms, antidepressants and disability and future coronary heart disease and stroke events in older adults: the Three City study. Eur J Epidemiol. 2013;28(3):249-56.
381. Rahman I, Humphreys K, Bennet AM, Ingelsson E, Pedersen NL, Magnusson PKE. Clinical depression, antidepressant use and risk of future cardiovascular disease. Eur $\mathrm{J}$ Epidemiol. 2013;28(7):589-95.

382. Smith GD, Hypponen E, Moldovan M, Power C. Death of siblings in childhood and subsequent mortality: prospective observational study. Eur J Epidemiol. 2014;29(11):859-61.

383. Timm S, Svanes C, Janson C, Sigsgaard T, Johannessen A, Gislason $\mathrm{T}$, et al. Place of upbringing in early childhood as related to inflammatory bowel diseases in adulthood: a population-based cohort study in Northern Europe. Eur J Epidemiol. 2014;29(6):429-37.

384. Westerlund A, Bellocco R, Sundstrom J, Adami HO, Akerstedt T, Lagerros YT. Sleep characteristics and cardiovascular events in a large Swedish cohort. Eur J Epidemiol. 2013;28(6):463-73.

385. Duijts L, Reiss I, Brusselle G, de Jongste J. Early origins of chronic obstructive lung diseases across the life course. Eur J Epidemiol. 2014;29(12):871-85.

386. Miller MR, Hankinson J, Brusasco V, Burgos F, Casaburi R, Coates A, et al. Standardisation of spirometry. Eur Respir J. 2005;26(2):319-38.

387. MacIntyre N, Crapo RO, Viegi G, Johnson DC, van der Grinten CPM, Brusasco V, et al. Standardisation of the single-breath determination of carbon monoxide uptake in the lung. Eur Respiratory J. 2005;26(4):720-35.

388. Hancock DB, Eijgelsheim M, Wilk JB, Gharib SA, Loehr LR, Marciante KD, et al. Meta-analyses of genome-wide association studies identify multiple loci associated with pulmonary function. Nat Genet. 2010;42(1):45-52.

389. Artigas MS, Loth DW, Wain LV, Gharib SA, Obeidat M, Tang WB, et al. Genome-wide association and large-scale follow up identifies 16 new loci influencing lung function. Nat Genet. 2011;43(11):1082-90.

390. Loth DW, Artigas MS, Gharib SA, Wain LV, Franceschini N, Koch B, et al. Genome-wide association analysis identifies six new loci associated with forced vital capacity. Nat Genet. 2014;46(7):669-77.

391. Brusselle G. Why doesn't reducing exacerbations decrease COPD mortality? Lancet Respir Med. 2014;2(9):681-3.

392. van Durme YM, Verhamme KM, Stijnen T, van Rooij FJ, Van Pottelberge GR, Hofman A, et al. Prevalence, incidence, and lifetime risk for the development of COPD in the elderly: the Rotterdam Study. Chest. 2009;135(2):368-77.

393. Lahousse L, van den Bouwhuijsen QJA, Loth DW, Joos GF, Hofman A, Witteman JCM, et al. Chronic obstructive pulmonary disease and lipid core carotid artery plaques in the elderly: the Rotterdam Study. Am J Respir Crit Care Med. 2013;187(1):58-64.

394. Lahousse L, Vernooij MW, Darweesh SKL, Akoudad S, Loth DW, Joos GF, et al. Chronic obstructive pulmonary disease and cerebral microbleeds. The Rotterdam Study. Am J Respir Crit Care Med. 2013;188(7):783-8.

395. Lahousse L, Verlinden VJA, van der Geest JN, Joos GF, Hofman A, Stricker BHC, et al. Gait patterns in COPD: the Rotterdam Study. Eur Respir J. 2015;19:2015.

396. Lahousse L, Niemeijer MN, van den Berg ME, Rijnbeek PR, Joos GF, Hofman A, et al. Chronic obstructive pulmonary disease and sudden cardiac death: the Rotterdam Study. Eur Heart J. 2015;36(27):1754-61.

397. Loth DW, Brusselle GG, Lahousse L, Hofman A, Leufkens HGM, Stricker BH. $\beta$-adrenoceptor blockers and pulmonary function in the general population: the Rotterdam Study. Br J Clin Pharmacol. 2014;77(1):190-200.

398. Luczynska A, Logan C, Nieters A, Elgizouli M, Schottker B, Brenner $\mathrm{H}$, et al. Cord blood 25(OH)D levels and the subsequent risk of lower respiratory tract infections in early 
childhood: the Ulm birth cohort. Eur J Epidemiol. 2014;29(8):585-94.

399. MacIntyre EA, Carlsten C, MacNutt M, Fuertes E, Melen E, Tiesler CMT, et al. Traffic, asthma and genetics: combining international birth cohort data to examine genetics as a mediator of traffic-related air pollution's impact on childhood asthma. Eur J Epidemiol. 2013;28(7):597-606.

400. Gnagnarella P, Maisonneuve P, Bellomi M, Rampinelli C, Bertolotti R, Spaggiari L, et al. Nutrient intake and nutrient patterns and risk of lung cancer among heavy smokers: results from the COSMOS screening study with annual low-dose CT. Eur J Epidemiol. 2013;28(6):503-11.

401. Loth DW, Ittermann T, Lahousse L, Hofman A, Leufkens HGM, Brusselle GG, et al. Normal spirometry values in healthy elderly: the Rotterdam Study. Eur J Epidemiol. 2013; 28(4):329-34.

402. Mohner M, Kersten N, Gellissen J. Diesel motor exhaust and lung cancer mortality: reanalysis of a cohort study in potash miners. Eur J Epidemiol. 2013;28(2):159-68.

403. Brostrom EB, Akre O, Katz-Salamon M, Jaraj D, Kaijser M. Obstructive pulmonary disease in old age among individuals born preterm. Eur J Epidemiol. 2013;28(1):79-85.

404. Kreiner-Moller E, Medina-Gomez C, Uitterlinden AG, Rivadeneira $F$, Estrada K. Improving accuracy of rare variant imputation with a two-step imputation approach. Eur J Hum Genet. 2015;23(3):395-400.

405. Boomsma DI, Wijmenga C, Slagboom EP, Swertz MA, Karssen LC, Abdellaoui A, et al. The genome of the Netherlands: design, and project goals. Eur J Hum Genet. 2014;22(2):221-7.

406. Westra HJ, Franke L. From genome to function by studying eQTLs. Biochim Biophys Acta. 2014;1842(10):1896-902.

407. Huan T, Esko T, Peters MJ, Pilling LC, Schramm K, Schurmann $\mathrm{C}$, et al. A meta-analysis of gene expression signatures of blood pressure and hypertension. PLoS Genet. 2015;11(3):e1005035.

408. Aleksandrova K, Jenab M, Bueno-de-Mesquita HB, Fedirko V, Kaaks R, Lukanova A, et al. Biomarker patterns of inflammatory and metabolic pathways are associated with risk of colorectal cancer: results from the European prospective investigation into cancer and nutrition (EPIC). Eur J Epidemiol. 2014;29(4): 261-75.

409. Altmaier E, Fobo G, Heier M, Thorand B, Meisinger C, Romisch-Margl W, et al. Metabolomics approach reveals effects of antihypertensives and lipid-lowering drugs on the human metabolism. Eur J Epidemiol. 2014;29(5):325-36.

410. Baltar VT, Xun WW, Johansson M, Ferrari P, Chuang SC, Relton $\mathrm{C}$, et al. A structural equation modelling approach to explore the role of $\mathrm{B}$ vitamins and immune markers in lung cancer risk. Eur J Epidemiol. 2013;28(8):677-88.

411. Broer L, Lill CM, Schuur M, Amin N, Roehr JT, Bertram L, et al. Distinguishing true from false positives in genomic studies: $p$ values. Eur J Epidemiol. 2013;28(2):131-8.

412. Broer L, Raschenberger J, Deelen J, Mangino M, Codd V, Pietilainen KH, et al. Association of adiponectin and leptin with relative telomere length in seven independent cohorts including 11,448 participants. Eur J Epidemiol. 2014;29(9):629-38.

413. Ege MJ, Strachan DP. Comparisons of power of statistical methods for gene-environment interaction analyses. Eur J Epidemiol. 2013;28(10):785-97.

414. Lind L, Elmstahl S, Bergman E, Englund M, Lindberg E, Michaelsson K, et al. EpiHealth: a large population-based cohort study for investigation of gene-lifestyle interactions in the pathogenesis of common diseases. Eur J Epidemiol. 2013;28(2): 189-97.

415. Mitrova E, Kosorinova D, Gajdos M, Sebekova K, Tomekova I. A pilot study of a genetic CJD risk factor (E200 K) in the general Slovak population. Eur J Epidemiol. 2014;29(8):595-7.
416. Raoult D, Foti B, Aboudharam G. Historical and geographical parallelism between the incidence of dental caries, Streptococcus mutans and sugar intake. Eur J Epidemiol. 2013;28(8):709-10.

417. Rydell M, Granath F, Cnattingius S, Magnusson C, Galanti MR. In-utero exposure to maternal smoking is not linked to tobacco use in adulthood after controlling for genetic and family influences: a Swedish sibling study. Eur J Epidemiol. 2014;29(7): 499-506.

418. Sjolander A, Lee W, Kallberg H, Pawitan Y. Bounds on sufficient-cause interaction. Eur J Epidemiol. 2014;29(11):813-20.

419. Stang A, Poole C. The researcher and the consultant: a dialogue on null hypothesis significance testing. Eur $\mathrm{J}$ Epidemiol. 2013;28(12):939-44.

420. Stang A, Poole $C$. The researcher and the consultant: a dialogue on null hypothesis significance testing (vol 28, pg 939, 2013). Eur J Epidemiol. 2014;29(3):225.

421. Steenstrup T, Hjelmborg JVB, Mortensen LH, Kimura M, Christensen K, Aviv A. Leukocyte telomere dynamics in the elderly. Eur J Epidemiol. 2013;28(2):181-7.

422. Thompson CA, Zhang ZF, Arah OA. Competing risk bias to explain the inverse relationship between smoking and malignant melanoma. Eur J Epidemiol. 2013;28(7):557-67.

423. VanderWeele TJ. Unmeasured confounding and hazard scales: sensitivity analysis for total, direct, and indirect effects. Eur J Epidemiol. 2013;28(2):113-7.

424. Verhulst S, Aviv A, Benetos A, Berenson GS, Kark JD. Do leukocyte telomere length dynamics depend on baseline telomere length? An analysis that corrects for 'regression to the mean'. Eur J Epidemiol. 2013;28(11):859-66.

425. Chowdhury R, Khan H, Heydon E, Shroufi A, Fahimi S, Moore $\mathrm{C}$, et al. Adherence to cardiovascular therapy: a meta-analysis of prevalence and clinical consequences. Eur Heart J. 2013;34(38):2940-8.

426. Postmus I, Trompet S, Deshmukh HA, Barnes MR, Li X, Warren HR, et al. Pharmacogenetic meta-analysis of genomewide association studies of LDL cholesterol response to statins. Nat Commun. 2014;5:5068.

427. de Keyser CE, Eijgelsheim M, Hofman A, Sijbrands EJ, Maitland-van der Zee AH, van Duijn CM, et al. Single nucleotide polymorphisms in genes that are associated with a modified response to statin therapy: the Rotterdam Study. Pharmacogenomics J. 2011;11(1):72-80.

428. de Keyser CE, Becker ML, Uitterlinden AG, Hofman A, Lous JJ, Elens L, et al. Genetic variation in the PPARA gene is associated with simvastatin-mediated cholesterol reduction in the Rotterdam Study. Pharmacogenomics. 2013;14(11):1295-304.

429. de Keyser CE, Becker ML, Hofman A, Lous JJ, Uitterlinden AG, Visser LE, et al. The rs13064411 polymorphism in the WDR52 gene, associated with PCSK9 levels, modifies statininduced changes in serum total and LDL cholesterol levels. Pharmacogenet Genomics. 2015;25(3):134-42.

430. Leusink M, de Keyser CE, Onland-Moret NC, Hofman A, Visser LE, Stricker BH, et al. No association between CYP3A4*22 and statin effectiveness in reducing the risk for myocardial infarction. Pharmacogenomics. 2014;15(11): 1471-7.

431. de Keyser CE, Peters BJ, Becker ML, Visser LE, Uitterlinden AG, Klungel $\mathrm{OH}$, et al. The SLCO1B1 c.521T $>$ C polymorphism is associated with dose decrease or switching during statin therapy in the Rotterdam Study. Pharmacogenet Genomics. 2014;24(1):43-51.

432. de Keyser CE, de Lima FV, de Jong FH, Hofman A, de Rijke $\mathrm{YB}$, Uitterlinden AG, et al. Use of statins is associated with lower serum total and non-sex hormone-binding globulin-bound testosterone levels in male participants of the Rotterdam Study. Eur J Endocrinol. 2015;173(2):155-65. 
433. Niemeijer MN, van den Berg ME, Leening MJ, Hofman A, Franco OH, Deckers JW, et al. Declining incidence of sudden cardiac death from 1990-2010 in a general middle-aged and elderly population: The Rotterdam Study. Heart Rhythm. 2015;12(1):123-9.

434. Arking DE, Pulit SL, Crotti L, van der Harst P, Munroe PB, Koopmann TT, et al. Genetic association study of QT interval highlights role for calcium signaling pathways in myocardial repolarization. Nat Genet. 2014;46(8):826-36.

435. Noordam R, van den Berg ME, Niemeijer MN, Aarts N, Leening MJ, Deckers JW, et al. Assessing prolongation of the heart rate corrected QT interval in users of tricyclic antidepressants: advice to use Fridericia rather than Bazett's correction. J Clin Psychopharmacol. 2015;35(3):260-5.

436. Maljuric NM, Noordam R, Aarts N, Niemeijer MN, van den Berg ME, Hofman A, et al. Use of selective serotonin reuptake inhibitors and the heart-rate corrected QT interval in a real-life setting: the population-based Rotterdam Study. Br J Clin Pharmacol. 2015. doi:10.1111/bcp.12681.

437. Niemeijer MN, van den Berg ME, Eijgelsheim M, van Herpen G, Stricker BH, Kors JA, et al. Short-term QT variability markers for the prediction of ventricular arrhythmias and sudden cardiac death: a systematic review. Heart. 2014;100(23):1831-6.

438. Krijthe BP, Heeringa J, Hofman A, Franco OH, Stricker BH. Non-steroidal anti-inflammatory drugs and the risk of atrial fibrillation: a population-based follow-up study. BMJ Open. 2014;4(4):e004059.

439. Krijthe BP, Heeringa J, Kors JA, Hofman A, Franco OH, Witteman JC, et al. Serum potassium levels and the risk of atrial fibrillation: the Rotterdam Study. Int J Cardiol. 2013;168(6): 5411-5.

440. Lubitz SA, Lunetta KL, Lin H, Arking DE, Trompet S, Li G, et al. Novel genetic markers associate with atrial fibrillation risk in Europeans and Japanese. J Am Coll Cardiol. 2014;63(12):1200-10.

441. Aarts N, Noordam R, Hofman A, Tiemeier H, Stricker BH, Visser LE. Utilization patterns of antidepressants between 1991 and 2011 in a population-based cohort of middle-aged and elderly. Eur Psychiatry. 2014;29(6):365-70.

442. Noordam R, Aarts N, Hofman A, van Schaik RH, Stricker BH, Visser LE. Association between genetic variation in the ABCB1 gene and switching, discontinuation, and dosage of antidepressant therapy: results from the Rotterdam Study. J Clin Psychopharmacol. 2013;33(4):546-50.

443. Aarts N, Akoudad S, Noordam R, Hofman A, Ikram MA, Stricker BH, et al. Inhibition of serotonin reuptake by antidepressants and cerebral microbleeds in the general population. Stroke. 2014;45(7):1951-7.

444. Akoudad S, Darweesh SK, Leening MJ, Koudstaal PJ, Hofman A, van der Lugt A, et al. Use of coumarin anticoagulants and cerebral microbleeds in the general population. Stroke. 2014;45(11):3436-9.

445. Darweesh SK, Leening MJ, Akoudad S, Loth DW, Hofman A, Ikram MA, et al. Clopidogrel use is associated with an increased prevalence of cerebral microbleeds in a stroke-free population: the Rotterdam Study. J Am Heart Assoc. 2013;2(5):e000359.

446. van Blijderveen JC, Verhamme KM, Zietse R, Visser LE, Romio SA, Buhre PN, et al. Overanticoagulation is associated with renal function decline. J Nephrol. 2013;26(4):691-8.

447. Rodenburg EM, Visser LE, Hoorn EJ, Ruiter R, Lous JJ, Hofman A, et al. Thiazides and the risk of hypokalemia in the general population. J Hypertens. 2014;32(10):2092-7 discussion

448. Levin D, Bell S, Sund R, Hartikainen SA, Tuomilehto J, Pukkala E, et al. Pioglitazone and bladder cancer risk: a multipopulation pooled, cumulative exposure analysis. Diabetologia. 2015;58(3):493-504.
449. Lundby A, Rossin EJ, Steffensen AB, Acha MR, Newton-Cheh $\mathrm{C}$, Pfeufer A, et al. Annotation of loci from genome-wide association studies using tissue-specific quantitative interaction proteomics. Nat Methods. 2014;11(8):868-74.

450. Sitlani CM, Rice KM, Lumley T, McKnight B, Cupples LA, Avery CL, et al. Generalized estimating equations for genomewide association studies using longitudinal phenotype data. Stat Med. 2015;34(1):118-30.

451. Noordam R, Direk N, Sitlani CM, Aarts N, Tiemeier H, Hofman $\mathrm{A}$, et al. Identifying genetic loci associated with antidepressant drug response with drug-gene interaction models in a population-based study. J Psychiatr Res. 2015;62:31-7.

452. Stricker BH, Stijnen T. Analysis of individual drug use as a time-varying determinant of exposure in prospective populationbased cohort studies. Eur J Epidemiol. 2010;25(4):245-51.

453. de Keyser CE, Leening MJ, Romio SA, Jukema JW, Hofman A, Ikram MA, et al. Comparing a marginal structural model with a Cox proportional hazard model to estimate the effect of timedependent drug use in observational studies: statin use for primary prevention of cardiovascular disease as an example from the Rotterdam Study. Eur J Epidemiol. 2014;29(11):841-50.

454. Ali MS, Groenwold RHH, Pestman WR, Belitser SV, Hoes AW, de Boer A, et al. Time-dependent propensity score and colliderstratification bias: an example of beta(2)-agonist use and the risk of coronary heart disease. Eur J Epidemiol. 2013;28(4):291-9.

455. Been JV, Sheikh A. Gestational age misclassification and its effect on disease outcomes after preterm birth. Eur J Epidemiol. 2013;28(6):525-6.

456. Bless PJ, Schmutz C, Suter K, Jost M, Hattendorf J, MausezahlFeuz M, et al. A tradition and an epidemic: determinants of the campylobacteriosis winter peak in Switzerland. Eur J Epidemiol. 2014;29(7):527-37.

457. Chang ET, Boffetta P, Adami HO, Cole P, Mandel JS. A critical review of the epidemiology of agent orange/TCDD and prostate cancer. Eur J Epidemiol. 2014;29(10):667-723.

458. Crump C, Sundquist J, Sieh W, Winkleby MA, Sundquist K. Perinatal risk factors for Wilms tumor in a Swedish national cohort. Eur J Epidemiol. 2014;29(3):191-7.

459. Edgren G, Lagiou P, Trichopoulos D, Adami HO. Screening, case finding or primary cancer prevention in the developing world? Eur J Epidemiol. 2013;28(4):287-90.

460. Galeotti F, Massari M, D'Alessandro R, Beghi E, Chio A, Logroscino $\mathrm{G}$, et al. Risk of Guillain-Barre, syndrome after 2010-2011 influenza vaccination. Eur J Epidemiol. 2013;28(5):433-44.

461. de Groot MCH, Klungel OH, Leufkens HGM, van Dijk L, Grobbee DE, van de Garde EMW. Sources of heterogeneity in case-control studies on associations between statins, ACE-inhibitors, and proton pump inhibitors and risk of pneumonia. Eur J Epidemiol. 2014;29(10):767-75.

462. Ivkovic V, Karanovic S, Prlic MF, Misic M, Kos J, Juric D, et al. Is herbal tea consumption a factor in endemic nephropathy? Eur J Epidemiol. 2014;29(3):221-4.

463. Lai SW, Liao KF, Lai HC, Muo CH, Sung FC, Chen PC. Statin use and risk of hepatocellular carcinoma. Eur J Epidemiol. 2013;28(6):485-92.

464. Ludvigsson JF, Zugna D, Cnattingius S, Richiardi L, Ekbom A, Ortqvist $\mathrm{A}$, et al. Influenza $\mathrm{H} 1 \mathrm{~N} 1$ vaccination and adverse pregnancy outcome. Eur J Epidemiol. 2013;28(7):579-88.

465. Nezvalova-Henriksen K, Spigset O, Nordeng H. Triptan safety during pregnancy: a Norwegian population registry study. Eur J Epidemiol. 2013;28(9):759-69.

466. Rezaeian M. Wars versus SARS: Are epidemiological studies biased? Eur J Epidemiol. 2014;29(6):453-4.

467. Smedts HPM, de Jonge L, Bandola SJG, Baardman ME, Bakker $\mathrm{MK}$, Stricker BHC, et al. Early pregnancy exposure to 
antihistamines and risk of congenital heart defects: results of two case-control studies. Eur J Epidemiol. 2014;29(9):653-61.

468. Stang A, Kaab-Sanyal V, Hense HW, Becker N, Kuss O. Effect of mammography screening on surgical treatment for breast cancer: a nationwide analysis of hospitalization rates in Germany 2005-2009. Eur J Epidemiol. 2013;28(8):689-96.

469. van der Lijn F, den Heijer T, Breteler MM, Niessen WJ. Hippocampus segmentation in MR images using atlas registration, voxel classification, and graph cuts. Neuroimage. 2008;43(4):708-20.

470. Vrooman HA, Cocosco CA, van der Lijn F, Stokking R, Ikram MA, Vernooij MW, et al. Multi-spectral brain tissue segmentation using automatically trained k-Nearest-Neighbor classification. Neuroimage. 2007;37(1):71-81.

471. den Heijer T, van der Lijn F, Koudstaal PJ, Hofman A, van der Lugt A, Krestin GP, et al. A 10-year follow-up of hippocampal volume on magnetic resonance imaging in early dementia and cognitive decline. Brain. 2010;133(Pt 4):1163-72.

472. Vernooij MW, van der Lugt A, Ikram MA, Wielopolski PA, Vrooman HA, Hofman A, et al. Total cerebral blood flow and total brain perfusion in the general population: the Rotterdam Scan Study. J Cereb Blood Flow Metab. 2008;28(2):412-9.

473. Poels MM, Ikram MA, Vernooij MW, Krestin GP, Hofman A, Niessen WJ, et al. Total cerebral blood flow in relation to cognitive function: the Rotterdam Scan Study. J Cereb Blood Flow Metab. 2008;28(10):1652-5.

474. Zonneveld HI, Loehrer EA, Hofman A, Niessen WJ, van der Lugt A, Krestin GP, et al. The bidirectional association between reduced cerebral blood flow and brain atrophy in the general population. J Cereb Blood Flow Metab. 2015. doi:10.1038/ jcbfm.2015.157.

475. Vernooij MW, Ikram MA, Wielopolski PA, Krestin GP, Breteler MM, van der Lugt A. Cerebral microbleeds: accelerated 3D T2* weighted GRE MR imaging versus conventional 2D T2*-weighted GRE MR imaging for detection. Radiology. 2008;248(1):272-7.

476. Poels MM, Vernooij MW, Ikram MA, Hofman A, Krestin GP, van der Lugt A, et al. Prevalence and risk factors of cerebral microbleeds: an update of the Rotterdam Scan Study. Stroke. 2010;41(10 Suppl):S103-6.

477. Vernooij MW, van der Lugt A, Ikram MA, Wielopolski PA, Niessen WJ, Hofman A, et al. Prevalence and risk factors of cerebral microbleeds: the Rotterdam Scan Study. Neurology. 2008;70(14):1208-14.

478. Poels MM, Ikram MA, van der Lugt A, Hofman A, Krestin GP, Breteler MM, et al. Incidence of cerebral microbleeds in the general population: the Rotterdam Scan Study. Stroke. 2011;42(3):656-61.

479. Lovelock CE, Cordonnier C, Naka H, Al-Shahi Salman R, Sudlow CL, Edinburgh Stroke Study G, et al. Antithrombotic drug use, cerebral microbleeds, and intracerebral hemorrhage: a systematic review of published and unpublished studies. Stroke. 2010;41(6):1222-8.

480. Vernooij MW, Haag MD, van der Lugt A, Hofman A, Krestin $\mathrm{GP}$, Stricker $\mathrm{BH}$, et al. Use of antithrombotic drugs and the presence of cerebral microbleeds: the Rotterdam Scan Study. Arch Neurol. 2009;66(6):714-20.

481. Akoudad S, Ikram MA, Koudstaal PJ, Hofman A, Niessen WJ, Greenberg SM, et al. Cerebral microbleeds are associated with the progression of ischemic vascular lesions. Cerebrovasc Dis. 2014;37(5):382-8.

482. Akoudad S, Portegies ML, Koudstaal PJ, Hofman A, van der Lugt A, Ikram MA, et al. Cerebral microbleeds are associated with an increased risk of stroke: the Rotterdam Study. Circulation. 2015;132(6):509-16.

483. Vernooij MW, de Groot M, van der Lugt A, Ikram MA, Krestin GP, Hofman A, et al. White matter atrophy and lesion formation explain the loss of structural integrity of white matter in aging. Neuroimage. 2008;43(3):470-7.

484. Vernooij MW, Ikram MA, Vrooman HA, Wielopolski PA, Krestin GP, Hofman A, et al. White matter microstructural integrity and cognitive function in a general elderly population. Arch Gen Psychiatry. 2009;66(5):545-53.

485. de Groot M, Vernooij MW, Klein S, Ikram MA, Vos FM, Smith $\mathrm{SM}$, et al. Improving alignment in tract-based spatial statistics: evaluation and optimization of image registration. Neuroimage. 2013;1(76):400-11.

486. de Groot M, Ikram MA, Akoudad S, Krestin GP, Hofman A, van der Lugt A, et al. Tract-specific white matter degeneration in aging: the Rotterdam Study. Alzheimers Dement. 2015;11(3):321-30.

487. Agatston AS, Janowitz WR, Hildner FJ, Zusmer NR, Viamonte M Jr, Detrano R. Quantification of coronary artery calcium using ultrafast computed tomography. J Am Coll Cardiol. 1990;15(4):827-32.

488. Odink AE, van der Lugt A, Hofman A, Hunink MG, Breteler MM, Krestin GP, et al. Association between calcification in the coronary arteries, aortic arch and carotid arteries: the Rotterdam Study. Atherosclerosis. 2007;193(2):408-13.

489. Odink AE, van der Lugt A, Hofman A, Hunink MG, Breteler MM, Krestin GP, et al. Risk factors for coronary, aortic arch and carotid calcification. The Rotterdam Study. J Hum Hypertens. 2010;24(2):86-92.

490. Elias-Smale SE, Odink AE, Wieberdink RG, Hofman A, Hunink MG, Krestin GP, et al. Carotid, aortic arch and coronary calcification are related to history of stroke: the Rotterdam Study. Atherosclerosis. 2010;212(2):656-60.

491. Bos D, Portegies ML, van der Lugt A, Bos MJ, Koudstaal PJ, Hofman A, et al. Intracranial carotid artery atherosclerosis and the risk of stroke in whites: the Rotterdam Study. JAMA Neurol. 2014;71(4):405-11.

492. Takaya N, Yuan C, Chu B, Saam T, Underhill H, Cai J, et al. Association between carotid plaque characteristics and subsequent ischemic cerebrovascular events: a prospective assessment with MRI-initial results. Stroke. 2006;37(3):818-23.

493. Yuan C, Mitsumori LM, Ferguson MS, Polissar NL, Echelard D, Ortiz G, et al. In vivo accuracy of multispectral magnetic resonance imaging for identifying lipid-rich necrotic cores and intraplaque hemorrhage in advanced human carotid plaques. Circulation. 2001;104(17):2051-6.

494. Trial North American Symptomatic Carotid Endarterectomy. Methods, patient characteristics, and progress. Stroke. 1991;22(6):711-20.

495. Selwaness M, van den Bouwhuijsen Q, van Onkelen RS, Hofman A, Franco OH, van der Lugt A, et al. Atherosclerotic plaque in the left carotid artery is more vulnerable than in the right. Stroke. 2014;45(11):3226-30.

496. van den Bouwhuijsen QJ, Bos D, Ikram MA, Hofman A, Krestin GP, Franco OH, et al. Coexistence of calcification, intraplaque hemorrhage and lipid core within the asymptomatic atherosclerotic carotid plaque: the Rotterdam Study. Cerebrovasc Dis. 2015;39(5-6):319-24.

497. Akoudad S, Ikram MA, Koudstaal PJ, Hofman A, van der Lugt A, Vernooij MW. Cerebral microbleeds and the risk of mortality in the general population. Eur J Epidemiol. 2013;28(10):815-21.

498. Hou LF, Lloyd-Jones DM, Ning HY, Huffman MD, Fornage M, $\mathrm{He} \mathrm{K}$, et al. White blood cell count in young adulthood and coronary artery calcification in early middle age: coronary artery risk development in young adults (CARDIA) study. Eur J Epidemiol. 2013;28(9):735-42.

499. Wahl S, Mohlenkamp S, Erbel R, Moebus S, Andrich S, Stang A, et al. Screening results for subclinical coronary artery calcification in asymptomatic individuals in relation to a detailed 
parental history of premature coronary heart disease. Eur $\mathrm{J}$ Epidemiol. 2013;28(4):301-10.

500. White T, El Marroun H, Nijs I, Schmidt M, van der Lugt A, Wielopolki PA, et al. Pediatric population-based neuroimaging and the generation $\mathrm{R}$ study: the intersection of developmental neuroscience and epidemiology. Eur J Epidemiol. 2013;28(1): 99-111.

501. Smits C, Kapteyn TS, Houtgast T. Development and validation of an automatic speech-in-noise screening test by telephone. Int J Audiol. 2004;43(1):15-28.

502. Weber KP, Aw ST, Todd MJ, McGarvie LA, Curthoys IS, Halmagyi GM. Head impulse test in unilateral vestibular loss: vestibulo-ocular reflex and catch-up saccades. Neurology. 2008;70(6):454-63.

503. Cox RM, Alexander GC. The international outcome inventory for hearing aids (IOI-HA): psychometric properties of the English version. Int J Audiol. 2002;41(1):30-5.
504. Newman CW, Sandridge SA, Bolek L. Development and psychometric adequacy of the screening version of the tinnitus handicap inventory. Otol Neurotol. 2008;29(3):276-81.

505. Ciorba A, Bianchini C, Pelucchi S, Pastore A. The impact of hearing loss on the quality of life of elderly adults. Clin Interv Aging. 2012;7:159-63.

506. Gurgel RK, Ward PD, Schwartz S, Norton MC, Foster NL, Tschanz JT. Relationship of hearing loss and dementia: a prospective, population-based study. Otol Neurotol. 2014;35(5): 775-81.

507. Lin FR, Ferrucci L, Metter EJ, An Y, Zonderman AB, Resnick SM. Hearing loss and cognition in the Baltimore longitudinal study of aging. Neuropsychology. 2011;25(6):763-70.

508. Iwasaki S, Yamasoba T. Dizziness and imbalance in the elderly: age-related decline in the vestibular system. Aging Dis. 2015;6(1):38-47. 